\title{
Sequence Comparison in Historical Linguistics
}

Die Beobachtung ist in Beziehung auf Entstehung neuer Formen aus früheren auf sprachlichem Gebiete leichter und in grösserem Maassstabe anzustellen, als auf dem der pflanzlichen und thierischen Organismen. Ausnahmsweise sind wir Sprachforscher hier einmal im Vortheile gegen die übrigen Naturforscher.

Die Darwinsche Theorie und die Sprachwissenschaft, 17

Schleicher (1863)

That there are many seemingly striking parallels between biological evolution and language change has long since been noted by linguists as well as biologists. Starting from the rise of language studies as a scientific discipline in the early $19^{\text {th }}$ century up to today scholars from both disciplines have repeatedly pointed to similarities between the respective research objects and models in biology and linguistics. It is therefore not surprising that these parallels have also lead to the transfer of methods between both disciplines. Thus, starting from the 1980s, biologists began to employ transformational grammars (Chomsky 1959), originally designed to model the syntax of natural languages, to address certain sequence analysis problems in biology (Searls 2002, Durbin et al. 1998 [2002]: 233). Some 20 years later, scholars began to employ biological methods for phylogenetic reconstruction to solve questions of genetic language classification (Gray and Atkinson 2003, Ringe et al. 2002), eventually triggering a 'quantitative turn' which lead to the establishment of a whole new branch of 'quantitative historical linguistics'. The key assumption of these new approaches is that the characteristic processes of language change and biological evolution are so similar that the methods designed for one discipline may also be used in the other one, despite the fact that the domains differ (Croft 2008: 225). 


\begin{tabular}{|l|l|l|}
\hline Aspects & Species & Languages \\
\hline \hline unit of heredity & gene & word \\
\hline replication & (asexual and sexual) reproduction & learning \\
\hline origination & cladogenesis & language splitting \\
\hline forces of change & natural selection and genetic drift & social selection and trends \\
\hline differentiation & treelike & treelike (?) \\
\hline
\end{tabular}

Table 4.1: Some apparent parallels between species and languages

The majority of the new methods in quantitative historical linguistics assumes a direct mapping of linguistic and biological entities. Table 4.1 lists some of the most common parallels between linguistics and biology which are often proposed in the literature. Thus, regarding the unit of heredity, the biological gene is usually set in analogy with the linguistic word, both being 'discrete heritable units' (Pagel 2009: 406). Replication of the heritable units is achieved via concrete mechanisms of reproduction in biological evolution and via learning in language history. From the perspective of origination, cladogenesis in biology is identified with language splitting in linguistics (ibid.). From the perspective of change, the driving forces of biological evolution, such as natural selection and genetic drift are compared with social selection and trends, eventually leading to language change (ibid.). Last not least, differentiation is usually assumed to be treelike, and the impact of 'horizontal forces' on evolution is considered to be rather low in both cases.

Assuming that these parallels hold, it seems perfectly plausible to use the methods developed for the application in one discipline in the other. However, it is important to be aware not only of the parallels but also of the differences between the research objects of both disciplines. The most striking difference between languages and genomes is that biological evolution manifests itself substantially while language history does not. In terms of Popper (1978), genome evolution and language evolution take place in different worlds: While biological organisms are part of world 1, the "world that consists of physical bodies' (ibid.: 143), languages belong to world 3, the 'world of the products 
of the human mind, such as languages; tales and stories and religious myths' (ibid.: 144). ${ }^{1}$

The new automatic methods are usually thought to have a greater degree of objectivity and reliability compared to the traditional framework of the comparative method (McMahon and McMahon 2005: 26-29), which is a rather intuitive enterprise (Schwink 1994: 29), suffering from a lack of probabilistic thinking (Baxter and Manaster Ramer 2000: 169-172). However, it is interesting to note that the most crucial part of the analysis, namely the identification of sound correspondences and cognates (steps 2 and 3 of the working procedure outlined in Section 2.6), is still almost exclusively carried out manually. That this may be problematic was recently shown in a comparison of two large lexicostatistical word lists produced by different scholarly teams where differences in item translation and cognate judgments led to topological differences of more than $30 \%$ in the automatically calculated phylogenetic trees (Geisler and List forthcoming). Thus, new automatic approaches do not necessarily lead to an overall increase in objectivity and reliability, as long as they are applied to datasets produced by the intuitive, qualitative methods they are supposed to overcome.

The goal of this study is to cope with this gap by developing a new framework for automatic sequence comparison in historical linguistics. Based on novel approaches to sequence modelling (Section 4.1) and phonetic alignment (Section 4.2) a new method for automatic cognate detection has been developed (Section 4.3), which automatically performs the relevant steps 2 and 3 of the traditional comparative method (see Section 2.6).

\subsection{Sequence Modelling}

When dealing with automatic sequence comparison in historical linguistics, it is important to be clear about the underlying sequence model. Phonetic sequences differ crucially from biological sequences in several respects. The segmentation of sequences into phonetic segments (see Section 3.1.1), for example, poses a problem of itself which is addressed in the fields of phonology and phonetics. The processes dominating in biological evolution and language history may also differ quite significantly. Thus, the unit of heredity in biology, the gene, is built from a set of universal characters which can be found

1 For a more detailed account on language as part of Popper's world 3, see Keller (1990: 164-174). 


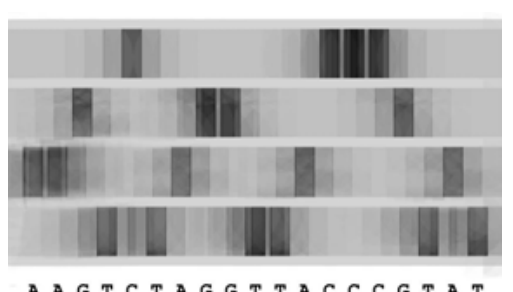

(a) Genes

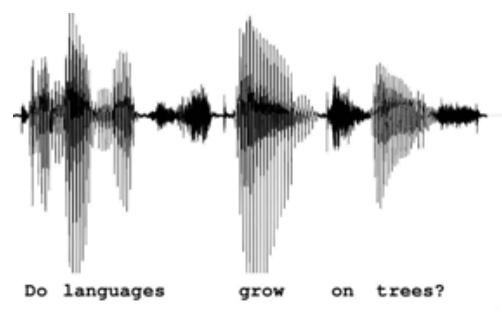

(b) Words

Figure 4.1: Discrete heritable units: genes and words. While the biological alphabet manifest itself substantially and can be retrieved by means of gel electrophoresis (a), the sounds of languages are physically only present as acoustic waves (b).

in all organisms. The unit of heredity in linguistics, the word, however, is built from a set of sounds which are distinct only with respect to the language they belong to (see Section 2.1.3). Unlike genes, words are not drawn from a universal alphabet, but from alphabets which themselves are subject to change (see Section 2.2.1). As a result, the problem of sequence alignment in historical linguistics differs fundamentally from the problem of sequence alignment in biology: While the latter requires only to find the best arrangement of two sequences in dependence of a scoring scheme that - once inferred - holds for all biological taxa, ${ }^{2}$ the former requires to find both the best arrangement of two words and the best scoring scheme which matches the sounds of the two languages from which the words are taken. ${ }^{3}$

In order to address these specific characteristics of linguistic sequences, new models for the representation of sequences were developed for this study. These new models cover both paradigmatic and syntagmatic aspects of sound sequences and play a crucial role in the algorithms for phonetic alignment and automatic cognate detection that are presented in this study. Paradigmatic aspects of sequence modelling focus on the comparison of sequences on the segment level. Syntagmatic aspects of sequence modelling, on the other hand,

2 In biology, different scoring schemes are used for different evolutionary distances, yet it is generally assumed that these scoring schemes apply globally to all biological taxa.

3 In the literature this problem is sometimes stated as a cryptanalytic problem where neither the mapping between source and target texts nor the mapping between source and target alphabets is known (Hoenigswald 1959). 
focus on the comparison of sequences with respect to their structure. All models are part of a specific framework for sequence modelling which is implemented as part of the LingPy Python library which was written for this study (see Supplementary Material).

\subsubsection{Paradigmatic Aspects}

\section{Sound Classes}

A strict notion of genotypic similarity (see Section 2.4.1) is prevalent in historical linguistics. Genotypic similarity is defined in absolute terms. Only if two segments are judged to correspond systematically, they are judged to be similar. As an example, consider the two words English mouth [mavð] and German Mund [mont] 'mouth'. From a genotypic perspective, these two words are maximally similar, since all correspondences, which are reflected in

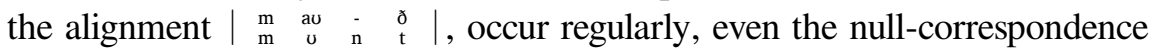
German [n] $\approx$ English [-] (Starostin 2010: 95). ${ }^{4}$ Once the language-specific genotypic similarities are identified for a pair of languages, it is not difficult to design an alignment algorithm that optimally aligns all genotypically similar words of these two languages, thereby rejecting all phonetically more similar candidates, such as, e.g., English mount [maunt], or German Maus [maus] 'mouse'. All one has to do is to design a specific scoring function which reflects the regular sound correspondences in a given language pair.

However, in the first stages of language comparison, neither the cognate words nor the sound correspondences are known. In order to find cognates and correspondences, we need a heuristic which helps us to find probably corresponding segments rather than absolutely corresponding ones. Many authors (Holzer 1996: 174f, Szemerényi 1970: 14f) emphasize that phenotypic similarity can be neglected when establishing correspondence patterns, since, "[...] given a long enough time span, almost any sound can change into any other sound" (Arlotto 1972: 77). While it is true that there are good examples for sound change which are difficult to explain on pure phonetic terms, most scholars, however, would probably also agree that sound change often does follow certain patters, that "[...] even the most divergent languages show examples of phonetic change which are remarkably similar" (ibid.), and that "[not] all changes are [...] equiprobable" (Lass 1997: 136). The difference

4 Compare, for example English other $[\Lambda$ ðə] $\approx$ German anders [andərs] 'different' and the

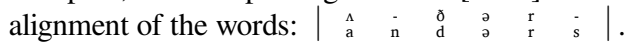


regarding the probability of certain sound changes to occur will also show up in the patterns of sound correspondences which can be observed in genetically related languages, with certain patterns occurring more often and other ones being quite rare. Stating segment similarity in terms of correspondence probability will differ from a pure phenotypic notion of similarity, yet it will, nevertheless, come closer to it than the strict notion of genotypic similarity, which is ignorant of phonetic realization.

The first attempt to derive an empirical model for the probability of sound correspondences that is known to me is an approach by A. B. Dolgopolsky (Dolgopolsky 1964). Based on partially empirical observations of soundcorrespondence frequencies in the languages of the world, which are - unfortunately - not further specified by the author, he divided speech sounds into ten types (see Table 4.2), and "[...] thereby distinguished [them] in such a way that phonetic correspondences inside a 'type' are more regular than those between different 'types"' (ibid.: 35). In a recent study, Dolgopolsky's soundclass model has been used as a heuristic device for automatic cognate detection (Turchin et al. 2010). According to this method, semantically identical basic words are judged to be cognate if their first two consonant sound classes match, otherwise, no cognacy is assumed. The advantage of this approach is that the number of false positives is usually considerably low (see the evaluation of the method in Section 4.3), the apparent disadvantage lies in the fact that many true positives are missed, since no true alignment analysis is carried out. Thus the cognate words German Tochter [toxtər] 'daughter' and English daughter [do:tər] do not match in their first two consonant classes ("TKTR" vs. "TTR"). An alignment analysis of the sound class strings, however, can

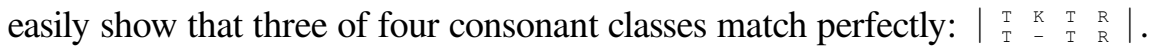

The advantage of sound class representations of phonetic segments compared to pure phonetic representations lies in the specific probabilistic notion of segment similarity inherent in the sound class approach. Offering a stochastically based intermediate solution between the two extreme positions of genotypic and phenotypic similarity, sound classes seem especially suitable for automatic sequence comparison. Choosing strings of sound classes as internal representation format has many advantages: While, as has been mentioned before, sequence comparison in disciplines such as evolutionary biology always deals with the same fixed set of characters, such as the protein or DNA alphabets, the sound systems of the world's languages differ to a great degree (compare the overview given in Maddieson 2011), and the number of 
characters (including diacritics) of phonetic transcription systems such as the IPA will force the algorithms to handle a large bunch of phonetic values which will be meaningless in most applications. This is due to the fact that, on the one hand, there is a lot of variation regarding the way linguists transcribe languages: Apart from the difference between narrow and broad phonetic transcriptions, there are many cases in which linguists simply slightly differ in their judgments, especially in poorly studied languages. On the other hand, there are many correspondence patterns which occur so frequently, that it seems justified to give the respective sounds an identical value from the beginning. Thus, while probably no one would doubt that the velar unvoiced plosive [k] should be kept distinct from the labial unvoiced plosive [p], the distinction between the velar nasal $[\mathrm{y}]$ and the uvular nasal [N] is far less obvious and it does not seem likely that the performance of any algorithm will suffer if both sounds will be merged into one.

In List (2012c), I proposed a revised version of Dolgopolsky's original sound-class model that was developed within a trial-and-error process when testing the suitability of different sound-class models for phonetic alignment. The Sound Class-Based Phonetic Alignment (SCA) model consists of 28 sound classes. In contrast to the model by Dolgopolsky (henceforth called DOLGO model), the SCA model is more fine-graded, distinguishing 16 consonant, six vowel, and six tone qualities (see Table 4.3). Among the consonant classes in

\begin{tabular}{|c|c|c|c|}
\hline No. & Cl. & Description & Examples \\
\hline 1 & "P " & labial obstruents & $p, b, f$ \\
\hline 2 & "T" & dental obstruents & $\mathrm{d}, \mathrm{t}, \theta, \partial$ \\
\hline 3 & "S " & sibilants & $\mathrm{s}, \mathrm{z}, \int, 3$ \\
\hline 4 & "K" & velar obstruents, dental and alveolar affricates & $k, g, t s, t f$ \\
\hline 5 & "M" & labial nasal & $\mathrm{m}$ \\
\hline 6 & "N" & remaining nasals & $\mathrm{n}, \mathrm{n}, \mathrm{y}$ \\
\hline 7 & "R" & liquids & $\mathrm{r}, \mathrm{l}$ \\
\hline 8 & "W" & voiced labial fricative and initial rounded vowels & $\mathrm{v}, \mathrm{u}$ \\
\hline 9 & "J" & palatal approximant & j \\
\hline 10 & "Ø" & laryngeals and initial velar nasal & $h, f, \eta$ \\
\hline
\end{tabular}

Table 4.2: Dolgopolsky's original sound class model 


\begin{tabular}{|c|c|c|c|}
\hline No. & Cl. & Description & Examples \\
\hline 1 & "A" & unrounded back vowels & $\mathrm{a}, \mathrm{a}$ \\
\hline 2 & "B" & labial fricatives & $\mathrm{f}, \beta$ \\
\hline 3 & "C" & dental / alveolar affricates & ts, d, t t , d \\
\hline 4 & "D" & dental fricatives & $\theta$, ð \\
\hline 5 & "E" & unrounded mid vowels & e, $\varepsilon$ \\
\hline 6 & "G" & velar and uvual fricatives & $\gamma, x$ \\
\hline 7 & "H " & laryngeals & $\mathrm{h}, ?$ \\
\hline 8 & "I " & unrounded close vowels & $\mathrm{i}, \mathrm{I}$ \\
\hline 9 & "J" & palatal approxoimant & $\mathrm{j}$ \\
\hline 10 & "K" & velar and uvular plosives & $\mathrm{k}, \mathrm{g}$ \\
\hline 11 & "L" & lateral approximants & 1 \\
\hline 12 & "M" & labial nasal & $\mathrm{m}$ \\
\hline 13 & "N" & nasals & $\mathrm{n}, \mathrm{\eta}$ \\
\hline 14 & "०" & rounded back vowels & $\mathrm{OE}, \mathrm{D}$ \\
\hline 15 & "P " & labial plosives & $\mathrm{p}, \mathrm{b}$ \\
\hline 16 & "R" & trills, taps, flaps & $\mathrm{r}$ \\
\hline 17 & "S " & sibilant fricatives & $\mathrm{s}, \mathrm{z}, \int, 3$ \\
\hline 18 & "T" & dental / alveolar plosives & $\mathrm{t}, \mathrm{d}$ \\
\hline 19 & "U" & rounded mid vowels & J, 0 \\
\hline 20 & "W" & labial approx. / fricative & $\mathrm{v}, \mathrm{w}$ \\
\hline 21 & "Y" & rounded front vowels & $\mathrm{u}, \mathrm{U}, \mathrm{y}$ \\
\hline 22 & "0 " & low even tones & 11,22 \\
\hline 23 & "1" & rising tones & 13,35 \\
\hline 24 & "2" & falling tones & 51,53 \\
\hline 25 & "3" & mid even tones & 33 \\
\hline 26 & " 4 " & high even tones & 44,55 \\
\hline 27 & " 5 " & short tones & 1,2 \\
\hline 28 & " 6 " & complex tones & 214 \\
\hline
\end{tabular}

Table 4.3: The SCA sound class model

the SCA model, the basic difference to the DOLGO model is the strict separation of the general sonority classes. Thus, affricates, plosives, and fricatives are now generally separated. The voicing distinction is still ignored, since voice 
is generally quite prone to assimilation and weakening, and many languages lack this distinction completely.

While sound classes in the original sense of Dolgopolsky are seldom applied in historical linguistics, the use of specific alphabets which reduce the large number of phonetic values attested in the languages of the world to smaller samples is quite common. The Automatic Similarity Judgment Program (ASJP), for example, uses a specific alphabet of 41 symbols (34 consonants, 7 vowels), called ASJP code (Brown et al. 2008), for its large collection of small word lists from more than 6000 languages of the world (Wichmann et al. 2010). In contrast to the DOLGO model, which was specifically designed for the purpose of historical comparison, the primary goal of the ASJP code was to ease the pain-staking labor of transcription, while at the same time reflecting the majority of most of the commonly occurring sounds of the languages of the world (Brown et al. 2008: 289). Nevertheless, since the ASJP code is also based on the idea to reduce the variation inherent in phonetic transcription in order to guarantee comparability, it fulfills the criterion of a sound-class model in the sense in which the term is used in this study. All three sound-class models (DOLGO, SCA, ASJP) that were discussed so far are implemented as basic models of sequence representation in the LingPy library. In order to guarantee the full applicability of the model, 6 tonal classes, originally not present, were added to the ASJP model, and the DOLGO model was extended by one class covering all vowels and one class covering all tones.

\section{Scoring Functions}

Dolgopolsky's original sound-class approach defined sound classes as absolute entities. Transitions between sound classes were not allowed, although they are surely desirable, since transitions between classes are well-known to every historical linguist. Transition probabilities between sound classes can be easily modeled in the scoring functions of alignment algorithms. Scoring functions can be based on empirical or theoretical approaches. Within empirical approaches, scoring functions are derived from studies on sound correspondence frequencies in the languages of the world. This approach has been applied in List (2012b) where a specific substitution matrix for the ASJP sound-class model was derived from a study on sound correspondence frequencies in the languages of the world as they are reflected in the ASJP database (Brown et al. 2013). 


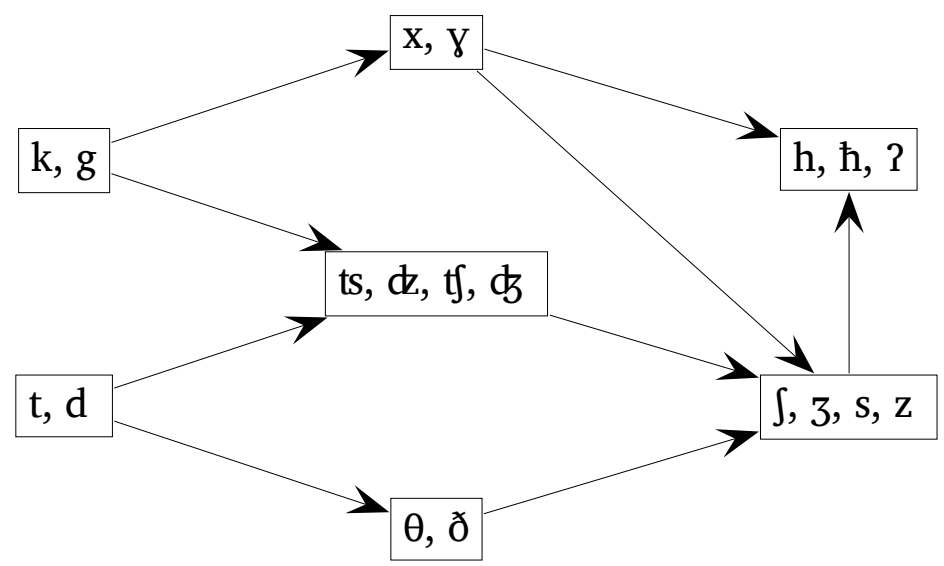

Figure 4.2: Directionality of sound change patterns

When deriving scoring functions from a theoretical basis, it is important to find a way to model the nature of sound change and sound correspondences. One crucial characteristic of certain well-known sound change types is their directionality, i.e. if certain sounds change, this change will go into a certain direction and the reverse change can rarely be attested. Other types of sound change are bidirectional and it cannot be decided which direction occurs more frequently. Thus, regarding velar plosives ( $[\mathrm{k}, \mathrm{g}])$, we know that they can be easily palatalized, and that palatalization consists of certain steps, where the velars first become affricates and then turn into sibilants (e.g. $[\mathrm{k}, \mathrm{g}]>[\mathrm{t}$, ts, $\left.\mathrm{b}, \mathrm{k}]>\left[\int, 3, \mathrm{z}, \mathrm{s}\right]\right)$. The same process of palatalization may happen with dental plosives (e.g. [t, d] $>[t]$, ts, $\mathrm{d}, \mathrm{d}]>\left[\int, 3, \mathrm{z}, \mathrm{s}\right]$ ). The opposite direction of these changes, however, is rarely attested. Another directional type of sound change which also occurs quite frequently is lenition whereby velar plosives become velar fricatives $([\mathrm{k}, \mathrm{g}]>[\mathrm{x}, \mathrm{y}]$ ), and dental plosives become dental fricatives $([t, d]>[\theta, \partial])$.

A direct consequence of the directionality of certain sound change types is that certain sound classes are very unlikely to occur in regular correspondence relations. This can be easily illustrated when displaying the possible transitions between sound classes resulting from common sound change types in a directed graph as it is done in Figure 4.2. As a rule, correspondence relations 
can only be assumed for those sound classes which either share a common ancestor, or are connected via a possible path through the graph. Hence, the correspondence $[\mathrm{x}, \mathrm{\gamma}] \approx[\mathrm{ts}, \mathrm{d}, \mathrm{t} f, \mathrm{~g}]$ is possible, as is the correspondence [ts, $\mathrm{d}, \mathrm{t} f, \mathrm{\Phi}] \approx[\theta, \delta]$. The correspondence $[\theta, \delta] \approx[\mathrm{x}, \mathrm{\gamma}]$, however is not possible (if the assumption of directionality holds), since there is no path which connects both sound classes. A scoring function which models the probability of sound classes to occur in correspondence relations should assign similarity scores which reflect the directionality of sound change types.

In order to define such a scoring function from a theoretical model, the following approach is applied: The scoring function is derived from a directed weighted graph. All sound classes which are known to be in very close connection to each other are connected by directed edges which reflect the direction of the respective sound changes. The assumed probability of the sound changes is defined by the edge weights. The higher the assumed probability of sound change, the smaller the weight. If sound change processes are not directional, both directions are reflected in the graph. This may, for example, be important for the scoring of vowels for which directional patterns of change are difficult to establish. The similarity score for two segments in the directed graph is calculated by subtracting the similarity score of one segment to itself from the length of the shortest path connecting two segments. In this context, the length of an edge in the directed graph is directly identified with the weight assigned to the edge. Figure 4.3 gives an example on how the similarity scores can be calculated for the above-mentioned cases of palatalization of dentals and ve-

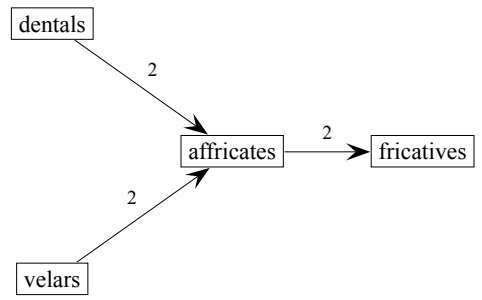

(a) Directed graph

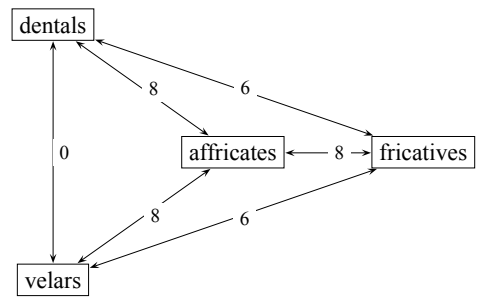

(b) Scoring scheme

Figure 4.3: Modelling the directionality of sound change in scoring functions. See the text for a detailed description. 
lars: The resulting similarity score for dentals and fricatives is calculated by subtracting the length of the shortest path (4) from the similarity score for a segment to itself (10). If no shortest path can be found, the similarity score is automatically set to 0 .

Based on the above stated, LingPy defines three different scoring functions for each of the basic sound-class models (DOLGO, SCA, and ASJP). The scoring function of the DOLGO model is the simplest one. It prohibits the matching of the vowel class with the consonant classes, and otherwise assigns high scores for identical sound classes. The SCA scoring function is based on the weighted-graph method. The underlying transition graph incorporates palatalization and lenition as exemplified above. Furthermore, some classes, such as the nasals and the glides, were given high similarity scores among each other, since transitions among these classes are quite likely as a result of assimilation. The ASJP scoring function is based on the empirical scoring function derived from the above-mentioned study on sound correspondence frequencies (Brown et al. 2013). ${ }^{5}$

The differences between the three sound-class models and their respective scoring functions can be illustrated by comparing the consonants in the sound system of English as it was defined by Chomsky and Halle (1968: 177). Figure 4.4 shows the results of a multidimensional scaling analysis for the different models. In this analysis, all sounds belonging to the same sound class in a given model were automatically clustered into one set. Furthermore, the distances between all sound sequences were calculated by converting the scoring functions (which reflect similarities) into distance scores. The distances in the model of Chomsky and Halle are derived by calculating the Hamming distances (see Section 3.1) of their feature vectors (ibid.). As can be seen from the figure, the Chomsky-Halle model of feature-based distances is not a good candidate for historical comparison. The DOLGO model receives its "historical strength" from the rough lumping of sound classes, but no further patterns are recognizable, since no explicit further transition probabilities between the sound classes are defined. Comparing the empirically derived ASJP model with the theoretically derived SCA model, one can find a lot of similarities, but even more striking differences. Thus, the voicing distinction, which was discarded in the SCA model, is only partially reflected in the ASJP model, and the closeness of nasals to their non-nasal counterparts in the ASJP model is completely absent in the SCA model.

5 Matrix representations of all three scoring functions are given in Appendix B. 
Chomsky \& Halle (1968)

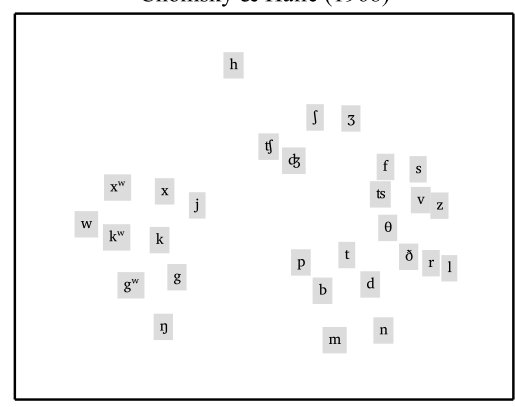

SCA (List 2012)

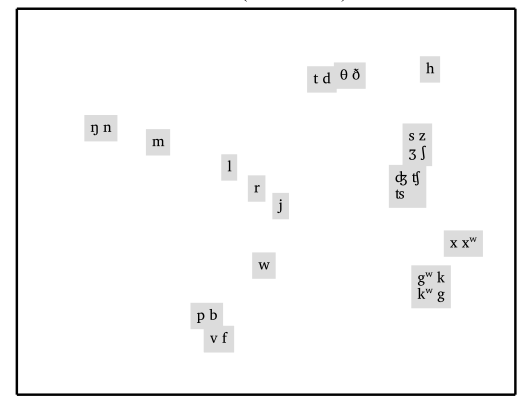

Dolgopolsky (1964)

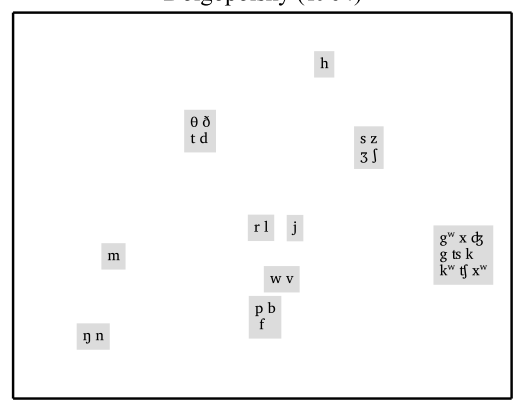

ASJP (Brown et al. 2013)

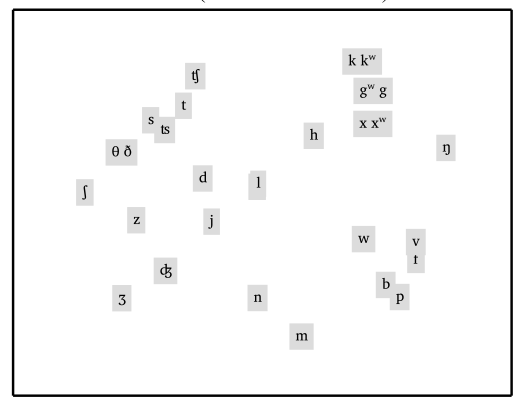

Figure 4.4: Multidimensional scaling analysis illustrating the distances between the sounds of English in different similarity models.

1

The differences between the different models are further visualized with the help of heat maps in Figure 4.5. Here, the fuzzy character of the Hamming distances derived from the features by Chomsky and Halle becomes especially evident. Of the larger clusters, only the velar group in the lower left of the matrices $\left(\left[\mathrm{x}^{\mathrm{w}}, \mathrm{k}^{\mathrm{w}}, \mathrm{g}^{\mathrm{w}}, \mathrm{x}, \mathrm{g}, \mathrm{w}\right]\right)$ finds a reflection in the other models. That the SCA model was derived from the DOLGO model can be easily spotted from a comparison of the respective matrices. Comparing the SCA with the ASJP model, the difference between empirical and theoretical models become quite obvious: While the ASJP matrix exhibits a broad range of partially very weak similarities between many different sound segments, the SCA model is much more restrictive. 


\subsubsection{Syntagmatic Aspects}

\section{Prosodic Context}

In biological sequence analyses it is common to treat specific positions of certain sequences differently by modifying the scores for the introduction of gaps (Thompson et al. 1994). In the approach presented here, the idea of position-specific scoring is adopted to incorporate syntagmatic information in the sound-class representation of phonetic sequences. The main idea behind this modification is to account for the well-known fact that certain types of sound change are more likely to occur in specific prosodic contexts. For example, vowels are more likely to get modified or lost than consonants, and
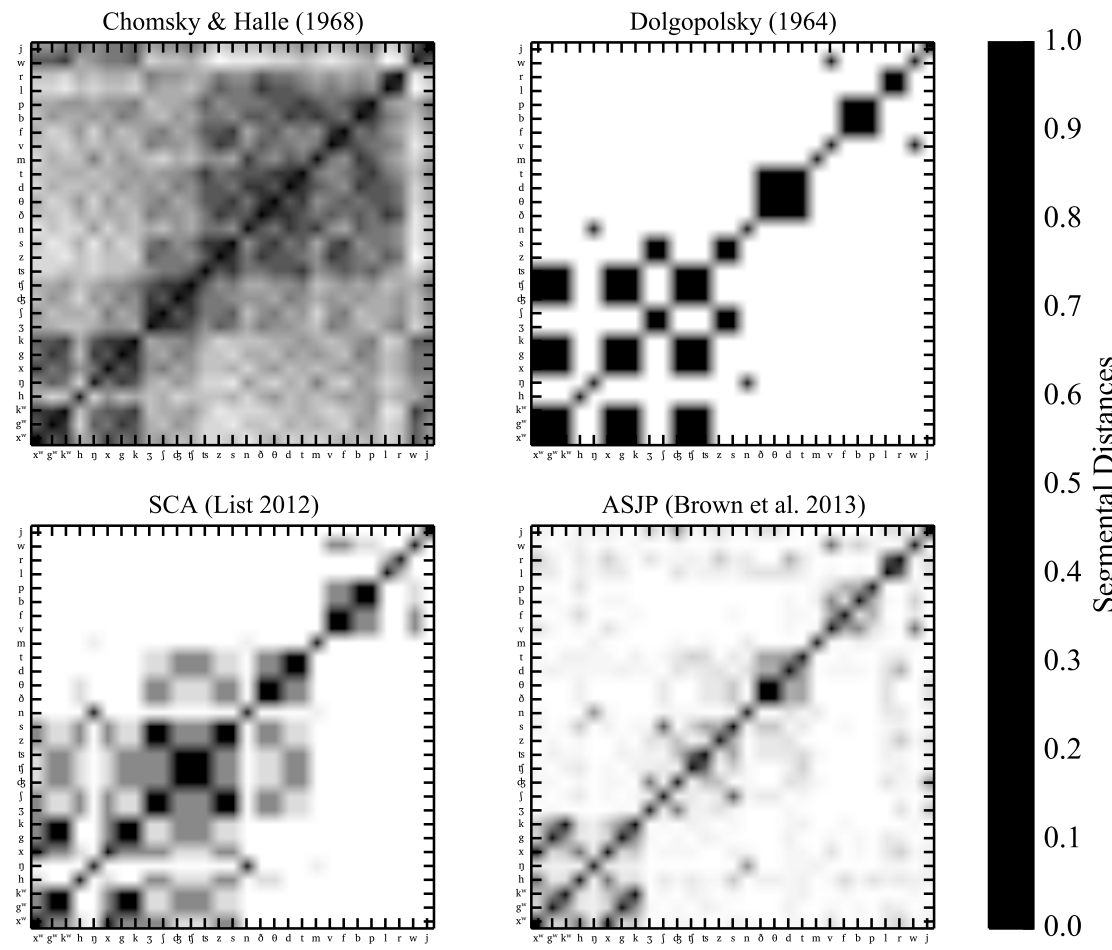

Figure 4.5: Distances between the sounds of English in different similarity models. 
consonants are more likely to get modified or lost in syllable-final than in syllable-initial position. It therefore seems fruitful to include this positionspecific information in the general representation of phonetic sequences. The information itself can then be used in different parts of sequence analyses. In alignment analyses, for example, it can be used to derive position-specific gap penalties (see Section 4.2). When searching for regular sound correspondences, the position-specific information offers an alternative to traditional $n$-gram representations of sequences (see Section 4.3).

The LingPy framework of sequence representation employs a very simple method to model prosodic context: A phonetic sequence is not only represented by its sound-class sequence, but also by a prosodic string, which itself is derived from the sequence's sonority profile. The sonority profile is represented as a vector of integer weights representing the relative sonority of all segments in a sonority hierarchy, going from lower weights for less sonorous segments to higher weights for more sonorous segments. LingPy currently employs the sonority hierarchy given in Example 4.1, which follows Geisler (1992: 30) with an additional sonority class for affricates:

$\begin{array}{ccccccc}\text { plosives } & \text { affricates } & \text { fricatives } & \text { nasals } & \text { liquids } & \text { glides } & \text { vowels } \\ 1 & 2 & 3 & 4 & 5 & 6 & 7\end{array}$

Once the sonority profile of a sequence is calculated, all segments can be assigned to different prosodic contexts according to their position in the profile. Given the fact that syllabification is often language-specific (Hall 2000: 226f), and that a given syllabification can become meaningless when multiple sequences are being compared, the framework for sequence modelling in LingPy employs a simplified strategy for the determination of prosodic context which is not based on syllabification. Given a sonority profile of a linguistic sequence, it is easy to determine peaks of maximum sonority, which will usually be represented by vowels. It is further possible to determine whether a given segment (which does not appear in a maximum peak position) is in a position of descending or ascending sonority. The derivation of these different prosodic contexts from a sonority profile is illustrated in Figure 4.6 for the sequence Bulgarian ябълка [jabəlka] 'apple'. Apart from the positions of ascending, descending, and maximum sonority, the word-initial and the word-final positions are treated as separate prosodic context, whereby a further distinction between vowels and consonants is being made. As a result, seven different prosodic contexts can be defined for phonetic segments, namely (1) "\#" - 


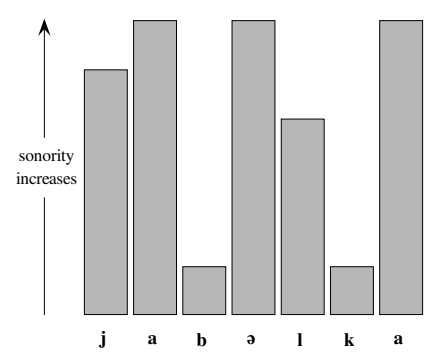

(a) Sonority profile

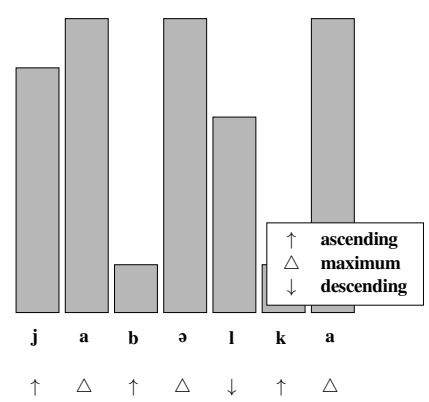

(b) Prosodic string

Figure 4.6: Deriving prosodic strings from sonority profiles. See the text for a detailed description.

word-initial ${ }^{6}$ consonants, (2) "V" - word-initial ${ }^{7}$ vowel, (3) "C" - ascending sonority, (4) "V" - sonority peak, (5) "C" - descending sonority, (6) "\$" word-final consonant, and (7) ">" - word-final vowel.

In order to derive position-specific weights, LingPy builds on an idea of Geisler (1992: 31-34) in assigning these contexts specific weights. These weights are defined in such a way that it is easier to introduce gaps in weak positions than in strong ones. The weights are represented as floating point numbers by which the GOP is multiplied. For example, a weight of 2.0 assigned to a specific environment will double the GOP for all segments occurring in this environment. The current default weights (see Table 4.4) have been determined by testing the performance of the SCA method for phonetic alignment (see Section 4.2) on a small representative sample of pairwise alignments. Two default sets are used: one for the alignment of South-East Asian tone languages, and one for all other languages. Using specific weights for tone languages is justified by the specific syllable structure of these languages that makes it necessary to assign high weights for vowels, and lower

6 This refers to all syllable-initial consonants in the first syllable of a word.

7 This refers to the first vowel in a word, regardless of whether it is preceded by a consonant or not. 


\begin{tabular}{|l|c|c|c|c|c|c|c|}
\hline \multirow{2}{*}{ Set of Weights } & \multicolumn{7}{|c|}{ Prosodic Context } \\
\cline { 2 - 8 } & $\#$ & $\mathrm{~V}$ & $\mathrm{C}$ & $\mathrm{C}$ & $\mathrm{V}$ & $\mathrm{S}$ & $>$ \\
\hline Tone L. & 1.6 & 3.0 & 1.2 & 1.1 & 3.0 & 0.5 & 0.7 \\
\hline Non-Tone L. & 2.0 & 1.5 & 1.5 & 1.1 & 1.3 & 0.8 & 0.7 \\
\hline
\end{tabular}

Table 4.4: The sets of default weights for tone and non-tone languages

weights for the position of descending sonority, since this position is seldom occupied.

The information regarding prosodic context is further used to modify the substitution scores slightly. They are increased if two segments belong to the same prosodic context. The amount by which the score is increased is also defined by a scaling factor, the default being 0.3 . Thus, if two segments match regarding their scaling factor, the resulting score is increased by $30 \%$. Table 4.5 summarizes the different representations of phonetic sequences applied by LingPy for Bulgarian $я б ъ л к а$ [jabəlka] 'apple'.

\section{Secondary Sequence Structures}

The idea of secondary sequence structures has been proposed in Section 3.3.3, and it is straightforward to apply this idea in an extended model for the rep-

\begin{tabular}{|l|ccccccc|}
\hline Phonetic Sequence & j & a & b & ə & l & k & a \\
\hline \hline SCA Model & J & A & P & E & L & K & A \\
\hline ASJP Model & V & a & b & I & l & k & a \\
\hline DOLGO Model & J & V & P & V & R & K & V \\
\hline Sonority Profile & 6 & 7 & 1 & 7 & 5 & 1 & 7 \\
\hline Prosodic String & \# & V & C & V & C & C & $>$ \\
\hline Relative Weight & 2.0 & 1.5 & 1.5 & 1.3 & 1.1 & 1.5 & 0.7 \\
\hline
\end{tabular}

Table 4.5: The LingPy framework of sequence modelling: Bulgarian ябълка 'apple' given in different representations. 
resentation of phonetic sequences. However, so far, the LingPy framework of sequence modelling is not capable to detect secondary sequence structures automatically. Therefore, only secondary structures which are explicitly marked as such by the user can be employed in the analyses. Thus, if a morpheme segmentation is included in the data, the alignment algorithm described in the next section, can carry out alignment analyses which are sensitive to secondary sequence structures. This is generally the case when dealing with South-East Asian tone languages, since the tone characters (which are represented by specific sound classes and are also assigned to a specific prosodic environment), automatically mark the syllable boundaries. Secondary alignment is especially useful in these cases, since the syllable in South-East Asian languages normally also reflects the morphemes. When aligning data containing sentences or phrases, the secondary structures (in form of white space characters) are usually also directly given along with the data. In all other cases, the data has to be manually or automatically preprocessed.

\subsection{Phonetic Alignment}

Although alignment analyses are the most general way to compare sequences manually and automatically, their application is still in its infancy in historical linguistics. Generally speaking, historical linguists have always aligned words. Without alignments, i.e. without the explicit matching of sounds, neither can regular sound correspondences be detected nor can cognacy between words or genetic relationship between languages be proven. However, although language comparison was always based on an implicit alignment of words, it was

\begin{tabular}{|l|llll|}
\hline Language & \multicolumn{4}{|l|}{ Alignment } \\
\hline \hline Choctaw & f & a & n & i \\
& & & & \\
Cree & - & i & 1 & u \\
\hline
\end{tabular}

(a) Pairwise alignment.

\begin{tabular}{|l|lllll|}
\hline Language & \multicolumn{4}{|l|}{ Alignment } & \\
\hline \hline Choctaw & - & $\mathrm{f}$ & $\mathrm{a}$ & $\mathrm{n}$ & $\mathrm{i}$ \\
Koasati & $\mathrm{i}$ & $\mathrm{p}$ & - & 1 & $\mathrm{u}$ \\
Cree & $\mathrm{i}$ & - & - & 1 & $\mathrm{u}$ \\
\hline
\end{tabular}

(b) Multiple alignment.

Table 4.6: The importance of multiple phonetic alignment (b) in comparison to pairwise phonetic alignment (a) illustrated for words meaning 'squirrel' in Muskogean (Fox 1995: 68, Haas 1969: 41). 


\begin{tabular}{|c|c|c|c|c|c|c|c|}
\hline Language & \multicolumn{7}{|c|}{ Alignment } \\
\hline Russian & $\mathrm{S}$ & - & J & $\mathrm{n}$ & ts & $\partial$ & - \\
\hline Polish & $\mathrm{s}$ & $\mathrm{w}$ & כ & $n^{j}$ & ts & $\varepsilon$ & - \\
\hline French & $\mathrm{S}$ & - & כ & 1 & - & $\varepsilon$ & $\mathrm{j}$ \\
\hline Italian & $\mathrm{S}$ & - & o & 1 & - & e & - \\
\hline German & $\mathrm{s}$ & - & כ & $\mathrm{n}$ & - & $\partial$ & - \\
\hline Swedish & $\mathrm{s}$ & - & u: & 1 & - & - & - \\
\hline
\end{tabular}

(a) Global alignment.

\begin{tabular}{|c|c|c|c|c|c|c|c|c|}
\hline Language & \multicolumn{8}{|c|}{ Alignment } \\
\hline Russian & $\mathrm{s}$ & כ & - & - & $\mathrm{n}$ & ts & ә & \\
\hline Polish & $\mathrm{S}$ & - & w & כ & $n^{j}$ & ts & $\varepsilon$ & \\
\hline French & s & ว & 1 & - & - & - & - & $\varepsilon j$ \\
\hline Italian & S & 0 & 1 & - & - & - & & $\mathrm{e}$ \\
\hline German & $S$ & ว & - & - & - & - & & nə \\
\hline Swedish & $s$ & u: & 1 & - & - & - & - & \\
\hline
\end{tabular}

(b) Local alignment.

Table 4.7: Two different alignments of reflexes of Proto-Indo-European *séh ${ }_{2}$ uel'sun'. In (a) all segments are globally aligned, resulting in an unrealistic scenario not reflecting known intermediate stages. In (b) all segments are locally aligned, whereby some elements are left unaligned, and the core block is shaded in gray. Segments connected via metathesis are displayed using a white font.

rarely explicitly visualized or termed as such, and in the rare cases where scholars explicitly used alignments to visualize correspondence patterns in words, it merely served illustrational purposes (Anttila 1972: 229-263, Lass 1997: 128).

That alignment analyses, and especially multiple alignment analyses, are important, is nicely illustrated by Fox (1995: 67f) in an example taken from Haas (1969: 41), where a pairwise comparison of Choctaw [fani] 'squirrel' and Cree [il] 'squirrel' leads to an incorrect matching of sound segments (see Table 4.6a), unless Koasati [iplu] 'squirrel' is added to the comparison (see Table 4.6b). It is, however, indicative that Fox does not use the term "alignment" but instead speaks of the problem of matching the correct "position in the string of phonemes" (Fox 1995: 67). This illustrates that linguists up to re- 
cently were unaware of the fact that the specific kind of sequence comparison they were carrying out had a close counterpart in other branches of science.

Another reason for this reluctance of linguists to make a broader use of alignment analyses may be that the representation of sequence differences with the help of alignments is not always apt to display the differences between phonetic sequences properly. Alignment analyses suggest a "holistic" picture of sequence differences where sequences are put into the matrix as a whole. As a result, it is difficult to display local similarities between sequences, especially when conducting multiple alignment analyses. As an example, consider ProtoIndo-European "séh, unel- 'sun' and its reflexes Russian солние [sontsə], Polish stońce [swon ${ }^{\mathrm{j}} \mathrm{ts \varepsilon}$ ], French soleil [solej], Italian sole [sole], German Sonne [sənə], Swedish sol [su:1] (NIL: 606f). A seemingly obvious global alignment of these words is given in Table 4.7a. This alignment, however, does only partially reflect the real correspondences between the words as they are proposed by the comparative method. The problem is that not all words are fully cognate, but have been derived indirectly via different morphological processes. Thus, French soleil goes back to Vulgar Latin *sōlǐculus 'small sun' (REW: § 8067), but Italian sole goes back to Latin sōlis 'sun’ (REW: §8059). Similarly, German Sonne and Swedish sol are usually assumed to be cognate, but their Proto-Germanic ancestor had a complex, stem-alternating paradigm. The Swedish word is a reflex of the nominative stem Proto-Germanic *sōel'sun', and the German word goes back to the oblique stem Proto-Germanic "sunnōn- (KROONEN: 463f). Russian солнще and Polish stońce both go back to Proto-Slavic *sülnbce 'sun' (DERKSEN: 479f), but the Proto-Slavic form cannot directly be traced back to Proto-Indo-European *séh $h_{2}$ uel-, but rather goes back to an early derivation * ${ }^{*} u h_{2} l-n$ - (NIL: 606). What further complicates an alignment of the words is the fact that the Polish word form results from a metathesis (Polish $[\mathrm{w}]<$ Proto-Slavic $* l$ ) which cannot directly be displayed in an alignment analysis. The primary purpose of alignment analyses in historical linguistics, but also in evolutionary biology, is to show which segments of different sequences are homologous. If we take this purpose seriously, the whole arrangement has to be rearranged drastically. In Table 4.7b, I have attempted to draw a more realistic scenario for the six words, leaving certain parts of the sequences unaligned, while at the same time using additional markup to display more specific relations.

Given the complexities and the problems to align remotely distant words realistically, it is surely not surprising that linguists so far have been reluctant 
to conduct such analyses. Nevertheless, even if it might not be possible at the moment to design algorithms which are accurate enough to yield an alignment as the one displayed in Table 4.7b, a careful adaptation of the methods that have been recently developed in evolutionary biology may yield alignments which come closer to the ideal than any linguist would probably have imagined 20 years ago.

\subsubsection{Previous Work}

When discussing previous work, one should distinguish implicit alignment algorithms, i.e. those approaches where alignment analyses have been used to calculate distances between phonetic sequences, and explicit alignment algorithms, i.e. those approaches where alignment analyses have been used as an explicit tool for the visualization of segment correspondences. The former studies, which started with an application by Kessler (1995) and since then have been followed up in many different applications, mostly in dialectology (see the overview in Nerbonne and Heeringa 2010), but also in large-scale genetic language comparison (Holman et al. 2011), are of less interest here, since the alignment method that I propose in the following is explicitly intended to serve the latter purpose.

When discussing explicit alignment algorithms, it is further important to distinguish between applications for pairwise phonetic alignment (PPA) and applications for multiple phonetic alignment. The first one to propose an explicit pairwise phonetic alignment algorithm was Covington (1996). The basic characteristics of this algorithm are a rough weighting scheme which especially prohibits the matching of vowels and consonants, and the use of affine gap costs. In contrast to later approaches proposed by other scholars, the algorithm is not based on dynamic programming, but on an extensive, partially guided, tree search of the whole space of possible alignments. In 1999, Somers reported an algorithm which was developed in the end of the 1970s and was originally intended to align narrow phonetic transcriptions of children's speech with a corresponding adult model in order to test the articulation of children. In contrast to the exhaustive tree search conducted by the algorithm of Covington, Somers's algorithm employed a greedy strategy which is not guaranteed to find the optimal pairwise alignment. The first PPA algorithm that employed dynamic programming was proposed by Oakes (2000). This algorithm was part of a larger framework (JAKARTA) that was intended to identify regular sound correspondences between language pairs. The align- 
ment algorithm in JAKARTA defines a considerably complex scoring scheme which reflects many different types of sound changes that are modeled by characterizing each sound segment with the help of three phonetic features (place, manner, voicing). Although JAKARTA defines a great number of different sound change types, the resulting distance function is rather rough, since the edit costs for divergent matches resulting from thesound change types are all set to 1 . Among the most sophisticated algorithms for PPA analyses is the ALINE algorithm (Kondrak 2000). Similar to JAKARTA, ALINE is also based on dynamic programming. Apart from the basic matching types, the algorithm has been extended to account for complex matches (compressions and expansions, see Section 3.2). Among the different alignment modes, global, local and semi-global alignment are supported. The most remarkable feature of ALINE, however, is the scoring function which is based on a multi-valued feature representation of phonetic segments and specific salience values for the features. The advantage of this approach is that the impact of features on similarity scores can be weighted according to their relevance for PPA analyses. Kondrak's ALINE algorithm was a milestone in phonetic alignment. Largely outperforming previously proposed algorithms (see the comparison of ALINE with other algorithms in Kondrak 2002: 64, also given in Table 4.12), it introduced many new concepts in phonetic alignment analyses, such as local and semi-global alignment. The only drawback of the algorithm is its restriction to pairwise alignment analyses.

Covington was also the first one to propose an explicit algorithm for multiple phonetic alignment (Covington 1998). Similar to his PPA algorithm, the MPA algorithm is based on a tree search instead of using dynamic programming. While this strategy may still work for PPA analyses, it is surely unsuitable for MPA analyses, as can be also seen from the fact that the author only gives examples for a maximum of three aligned sequences. More than 10 years later, Prokić et al. (2009) employed the ALPHAMALIG algorithm (Alonso et al. 2004) to carry out MPA analyses of a dataset containing the translational equivalents of 152 glosses in 192 Bulgarian dialects (Buldialect). Although the algorithm was not specifically designed for the task of phonetic alignment, Prokić et al. (2009) report a high accuracy of the performance of the algorithm in comparison with their manually edited gold standard. 


\begin{tabular}{|c|c|c|c|}
\hline INPUT & TOKENIZATION & CONVERSION & \\
\hline $\begin{array}{l}\text { toxtər } \\
\text { də:tər }\end{array}$ & $\begin{array}{l}\rightarrow \mathrm{t}, \mathrm{\partial}, \mathrm{x}, \mathrm{t}, \partial, \mathrm{r} \\
\mathrm{d}, \mathrm{\partial :}, \mathrm{t}, \partial, \mathrm{r}\end{array}$ & $\begin{array}{llllll}\mathrm{t} \partial \mathrm{X} \ldots & \rightarrow \mathrm{T} & \bigcirc & \mathrm{G} & \ldots \\
\mathrm{d} \partial \mathrm{t} \ldots & \rightarrow \mathrm{T} & \circ & \mathrm{T} & \ldots\end{array}$ & \\
\hline & ALIGNMENT & CONVERSION & OUTPUT \\
\hline & $\begin{array}{llllll}T & O & G & T & E & R \\
T & O & - & T & E & R\end{array}$ & $\begin{array}{l}\mathrm{T} \bigcirc \mathrm{G} \ldots \rightarrow \mathrm{t} \supset \mathrm{X} \ldots \\
\mathrm{T} \bigcirc-\ldots \rightarrow \mathrm{do:}-\ldots\end{array}$ & $\begin{array}{llllll}\mathrm{t} & \mathrm{J} & \mathrm{x} & \mathrm{t} & \partial & \mathrm{r} \\
\mathrm{d} & \partial: & - & \mathrm{t} & \partial & \mathrm{r}\end{array}$ \\
\hline
\end{tabular}

Figure 4.7: The basic working procedure of SCA analyses

\subsubsection{SCA - Sound-Class Based Phonetic Alignment}

The Sound-Class-Based Phonetic Alignment (SCA) method for pairwise and multiple phonetic alignment analyses was first introduced in List (2010), and successively revised in (List 2012b) and List (2012c). In the following, the most recent version of the method shall be introduced in detail. The SCA method combines both the current state of the art in general sequence alignment as it was outlined in Chapter 3 and the new approaches to sequence modelling that were presented in Section 4.1. The method is also implemented as part of the LingPy library (see Supplementary Material).

The main idea of the current version of SCA and all its predecessors is to distinguish between an external and an internal representation of phonetic sequences. The transition between the two representations is managed within a specific module which manages the conversion from external to internal format and vice versa. While the difference between external and internal format was merely a result of the conversion of IPA characters into capital letters reflecting sound-class models in the first version of SCA (List 2010), the current internal representation is a bit more complex, involving not only sound classes (see Section 4.1.1), but also prosodic strings (see Section 4.1.2). Distinguishing the external and internal representation of phonetic sequences has several advantages. Not only are the computational aspects easier to handle when working with a unified internal representation, the program is also much more flexible regarding the models that can be chosen for a given analysis. Since - from a technical viewpoint - sound classes merely constitute a substitution framework that accounts for the conversion of sequences drawn from one alphabet into sequences drawn from another one, there is no limitation regarding detail or roughness. One can conduct analyses where sounds are lumped into 
just a few general classes as proposed by Dolgopolsky (1964), as well as analyses which are virtually identical with narrow phonetic transcriptions.

Given that the IPA is chosen as the basic external representation format for phonetic sequences, a module in which IPA sequences are tokenized into meaningful phonetic segments precedes the first conversion stage. Hence, the basic working procedure of SCA consists of four stages: (1) tokenization, (2) class conversion, (3) alignment analysis, and (4) IPA conversion. In stage (1) the input sequences are tokenized into phonetic segments. In stage (2) the segments are converted into their internal representation format, whereas each sequence is further represented by its corresponding sound class sequence and its prosodic profile. The pairwise or multiple alignment analysis is carried out in stage (3). After the alignment analysis has been carried out, the aligned sequences are converted back to their original format in stage (4). This procedure is illustrated in Figure 4.7 for the words German Tochter [toxtər] 'daughter' and English daughter [də:tər].

\section{Pairwise Alignment}

The SCA method supports all structural extensions to the basic algorithm for pairwise sequence alignment mentioned in Section 3.3.2, including the extension for secondary alignment. When carrying out secondary alignment analyses, the boundary marker has to be defined by the user. By default, there are two different boundary markers: (1) tone letters as they are used in the system of phonetic transcriptions of Sinitic languages proposed by Chao (1930 [2006]), and (2) a marker for word boundaries, such as, e.g. the character "\#". Substitution scores are based on the respective sound-class model which is being used. All three models mentioned in Section 4.1.1 are available (DOLGO, SCA, ASJP), but more models can be easily defined by the user. The respective substitution matrices are derived as described in the same section. All analyses also support the use of position-specific gap and substitution scores derived from prosodic strings as described in Section 4.1.2. The relative weights for the different prosodic contexts can be defined by the user.

\section{Multiple Alignment}

The SCA algorithm for multiple phonetic alignment is based on the progressive alignment paradigm. In order to cope for the known problems of progres- 


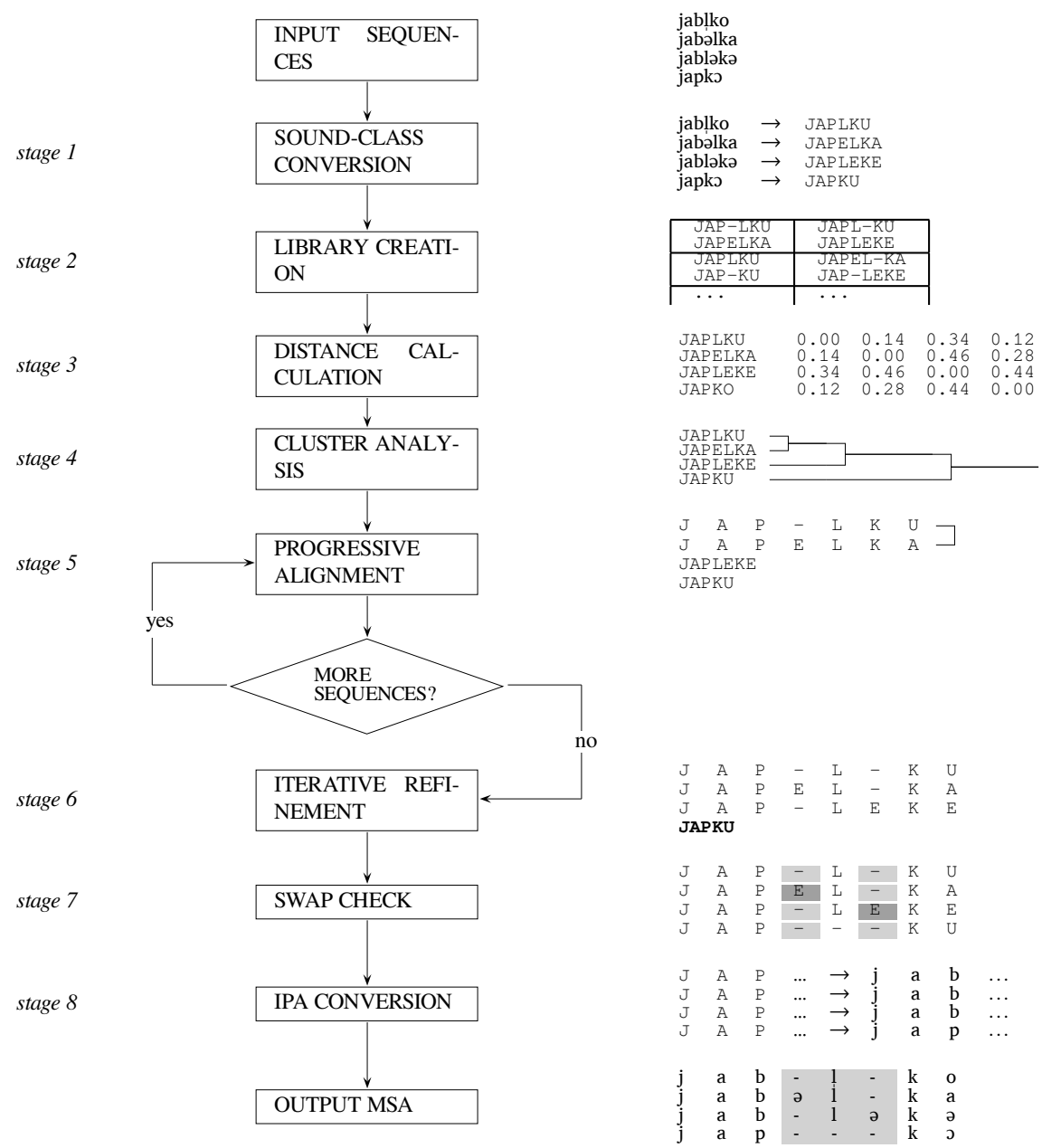

Figure 4.8: Working procedure of the SCA method. See the text for a further description of each of the eight stages.

sive algorithms, SCA makes use of pre- and postprocessing methods. For the preprocessing, SCA employs consistency-based scoring schemes. The postprocessing is based on iterative refinement (see Section 3.4.2). The working procedure of SCA consists of 8 stages as illustrated in Figure 4.8. In the figure, 
every stage is accompanied by an example which shows the current progress of the alignment of the four words Czech jablko [jablko], Bulgarian ябълка [jabəlka], Russian яблоко [jabləkə] and Polish jabłko [japko] 'apple'.

In stage 1, the input sequences which are converted to sound classes, e.g. the word Czech jablko is converted to "JAPLKU" according to the SCA model. In stage 2, the library of pairwise alignments is created. As a default, global, local, and diagonal alignment analyses (see Section 3.3.2) are carried out of all possible word pairs. In stage 3, the distance matrix is computed from the pairwise similarity scores of all sound class sequences. The conversion of similarity into distance scores is carried out with the formula of Downey et al. (2008):

$$
D=1-\frac{2 \cdot S_{A B}}{S_{A}+S_{B}}
$$

where $S_{A}$ and $S_{B}$ are the similarity scores of the sequences aligned with themselves, and $S_{A B}$ is the similarity score of the alignment of both sequences. In stage 4, the sound-class strings are clustered with help of the Neighbor-Joining algorithm (Saitou and Nei 1987). The clustering procedure yields the guide tree that is used for the progressive alignment in stage 5, where all sequences are stepwise aligned with each other, following the branching order of the guide tree. In stage 6, iterative refinement methods are applied to the already aligned sequences in order to account for possible errors resulting from misaligned sequences. In stage 7, a method for swap detection (described in detail in Section 4.2.3) is applied. As can be seen from the example, the algorithm correctly identifies a swap for the alignment of the four input sequences. In stage 8 , all sequences are converted from their internal representation back to their original IPA format.

\subsubsection{Specific Features}

\section{Iterative Refinement}

The basic principle of iterative refinement methods was described in Section 3.4.2. Since alignments in linguistics are much shorter than in biology, the heuristics for the partitioning of alignments in biology are not particularly apt for phonetic alignment. Therefore, three new strategies for alignment partitioning were developed for the SCA method, one single-type partitioning strategy, and two multi-type partitioning strategies. They may be called the 
orphan partitioning, flat-cluster partitioning, and similar-gap-sites partitioning strategy, respectively.

The (single-type) orphan partitioning strategy, ${ }^{8}$ splits and realigns the most divergent sequences from the MSA, whereas divergence is measured by taking the average distance of each sequence to all other sequences in the distance matrix. The flat-cluster partitioning strategy is based on a flat-cluster analysis of the data, using a flat-cluster variant of the UPGMA algorithm (Sokal and Michener 1958). In contrast to a traditional cluster analysis, the flat-cluster analysis stops to separate sequences when a certain threshold is reached. As a result, the set of input sequences in an alignment may be divided into a couple of subsets. The flat-cluster partitioning strategy for iterative refinement continuously splits off all sequences belonging to one of the flat clusters and realigns them, until all clusters have been split off and realigned once. The similargap-sites partitioning strategy also clusters the data into subsets, but not on the basis of sequence similarity, but on the basis of the current alignment. In an alignment, all rows can be assigned to a certain gap profile, depending on the positions at which they contain gaps. The similar-gap-sites partitioning strategy simply assigns all sequences to the same cluster, depending on whether they share an identical gap profile or not. Once the clusters are determined, the clusters are treated in the same way as in the flat-cluster partitioning strategy, i.e. each cluster is split off and realigned, until all clusters have been split off and realigned once.

\section{Consistency-Based Scoring}

Apart from traditional, matrix-based progressive alignment analyses, the SCA method also allows to carry out consistency-based alignment analyses as described in section 3.4.2. As mentioned before, the basic idea of consistencybased alignment is to use the information given in pairwise alignment analyses of the data to derive an alignment-specific scoring matrix. In the initial stage, the substitution scores for all residue pairs in the scoring matrix are set to 0 . After the pairwise alignments have been carried out, the scoring matrix is extended by increasing the score for each residue pair which occurs in the primary library by a certain weight. While in consistency-based methods in biology, such as, e.g. the T-Coffee algorithm (Notredame et al. 2000), the

8 In evolutionary biology, the term orphan is used to refer to "distant members of a [protein] family" (Thompson et al. 1999: 2684). 
weights for the substitution matrix are derived from percentage identity (see Equation 3.1 on page 74), the SCA method employs a different strategy, since, in contrast to biology, sequence identity is not a reliable measure in linguistics. Given the sequences $A$ and $B$, the weight $W_{x y}$ for the residues $x$ and $y$ being matched in an alignment of $A$ and $B$ is derived by the formula:

$$
W_{x y}=\frac{1}{2} \cdot\left(\frac{S_{A B}}{L_{A B}}+M_{x y}\right),
$$

where $S_{A B}$ is the similarity score of the alignment of $A$ and $B, L_{A B}$ is the length of the alignment, and $M_{x y}$ is the original substitution score for the residues. This equation combines both the general similarity of the aligned sequences and the individual similarity of the residue pair as defined by the scoring function. Apart from this modified weight formula, the consistencybased alignment of the SCA method does not differ from the description given in Section 3.4.2.

\section{Detection of Swapped Sites}

Swaps (crossed matches, see Section 3.2.1) are ignored in most approaches to phonetic alignment that have been proposed so far (Heeringa et al. 2006, Kessler 1995, Kondrak 2002, Prokić et al. 2009). Authors usually justify this ignorance for crossed matches by emphasizing the rareness of sound changes involving metathesis (Kondrak 2002: 50). A further problem is that it is quite difficult to extend the Needleman-Wunsch algorithm to account for transpositions, especially when dealing with specific scoring matrices instead of simple edit operations (Nerbonne and Heeringa 2010: 557). It may, however, nevertheless be useful to have a procedure that deals with swaps, since metathesis is not so rare as it is often claimed and it even regularly occurs in some languages (Hock 1991: 110).

The SCA strategy to deal with swaps differs from previous, formal solutions to the problem (Lowrance and Wagner 1975, Oommen and Loke 1997, Wagner 1975), in so far as it is not part of the basic alignment algorithm but a posterior procedure that is applied to already conducted multiple and pairwise alignment analyses. The basic idea of the method is to make use of the fact that the alignment of swaps in phonetic sequences often follows a similar pattern.

Given two hypothetical sequences "FORMA" and "FROMA", we may align and score them in different ways. When allowing for swaps, the Damerau- 

(a) \begin{tabular}{llllll}
$F$ & $O$ & $R$ & $M$ & $A$ \\
$F$ & $R$ & 0 & $M$ & $A$ \\
\hline \hline & 1 & 1 & 0 & 0
\end{tabular}
(b) \begin{tabular}{l|lllll}
$F$ & 0 & $R$ & $M$ & $A$ \\
$F$ & $R$ & 0 & $M$ & $A$ \\
\hline \hline & 1 & 0 & 1 & 0 & 0
\end{tabular}

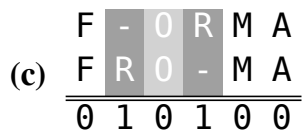

Figure 4.9: Different representations of swaps in alignment analyses

Levenshtein distance (named after the work of F. J. Damerau and V. I. Levenshtein, cf. Damerau 1964 and Levenshtein 1965) can be used. It builds on the edit distance (cf. Table 3.2), but it provides an additional penalty of 1 for crossed matches. A gap-free alignment of "FORMA" with "FROMA" will therefore yield a total score of 1 , since the sequences only differ by one transposition (see Figure 4.9a). Once we exclude transpositions in the scoring scheme, we arrive at a score of 2 . With a different scoring scheme where gaps are scored as 1, but mismatches are scored as 2, we can force the algorithm to avoid mismatches by spreading the swapped region over three columns instead of two (see Figure $4.9 b$ and c).

This is exactly the situation we find in most phonetic alignments, where swapped regions in sequences are aligned over three columns instead of being crushed into one. The usual alignment of Russian яблоко [jabləkə] and

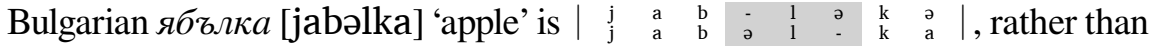
$\left|\begin{array}{ccccccc}j & a & b & 1 & a & k & a \\ j & a & b & a & 1 & k & a\end{array}\right|$. This is due to the fact that most phonetic alignment approaches, including the one presented in this study, disfavor the matching of consonants and vowels. Since most cases of metathesis involve the transposition of vowels and consonants, the natural representation of swaps in phonetic alignment analyses is the one that spreads them over three columns.

Detecting complementary columns in a given alignment is not difficult, and the computational effort is also rather low, since phonetic alignments are usually very short. Regions containing complementary columns, however, do not necessarily contain swaps. The easiest way to test whether complementary regions really point to swaps in an alignment is to calculate a swap score (an alignment score that allows for swaps), and to compare it with the original score: if the swap-score is better (higher in case of similarities, lower in case of distances), the swap is confirmed, if not, it is rejected.

There are surely many different ways to calculate an alignment score which allows for swaps. Within the SCA approach, the calculation of swap-scores 

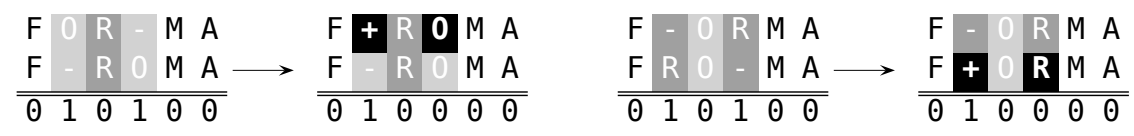

Figure 4.10: Calculating the swap-score with help of a trace character

is done by introducing a trace character ("+") into the sound class alphabet. This character scores only when it is matched with a gap, the score being the swap penalty. When matched with itself the trace character scores 0 , and it scores $\infty$ (or $-\infty$ when dealing with similarities) when being matched with any other character. The character is introduced into the gapped columns of a complementary site instead of all non-gap characters. The characters themselves are moved to the position where they would appear, if there was no swap in the alignment. The trace character "+" thus moves the original character by inducing a swap penalty at the same time. When transforming a given alignment in this way, the resulting score is identical with the Damerau-Levenshtein distance (if the swap penalty is set to 1 ), yielding 1 for two similar sequences which only differ by one transposition (see Figure 4.10).

One specific advantage of this procedure compared to the formal approaches to swap detection is that it works for pairwise as well as multiple alignments. Being a posterior method, it has the further advantage that it does not influence the computational complexity of the basic algorithm. It is left to the user to decide whether a search for swapped regions should be carried out in addition to the basic alignment analysis, or not.

The method for swap detection is illustrated in Figure 4.11 for the alignment of the four cognate words Czech žlutý [3lvti:], Russian жёлтый [3oltij], Polish żótty [3oltij], and Bulgarian жълm [3rlt] 'yellow' (DERKSEN: 565). In stage (1), the alignment is searched for complementary sites and the sum-ofpairs score is calculated. In stage (2), two copies of the alignment are created, one which is identical with the original one, and one in which the three columns of the probably swapped region are shifted in such a way that the outer columns appear as the inner column and the inner column is split into two outer ones. In stage (3), the trace character is inserted in the rightmost column and the rightmost non-gap characters are inserted in the first column. In stage (4), the sum-of-pairs scores for both new alignments are calculated. The comparison 


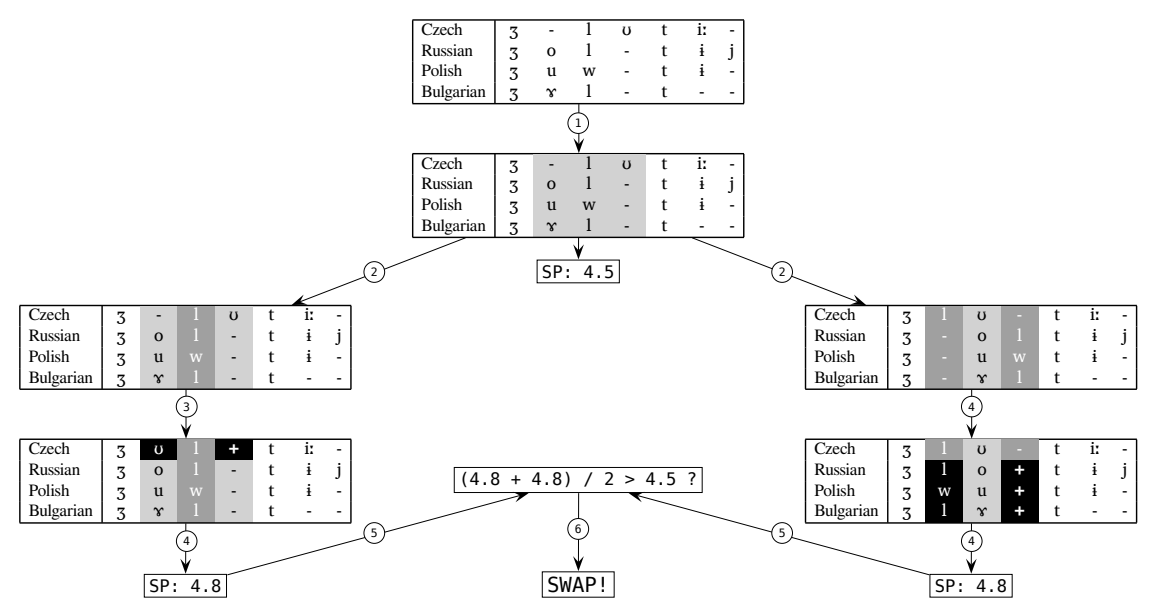

Figure 4.11: SCA method for the detection of swapped sites

of the average of the new scores with the original score in stage (5) yields the final decision in stage (6).

\subsubsection{Evaluation}

\section{Gold Standard}

Since automatic sequence comparison in historical linguistics is still in its infancy, the number of benchmark databases which are available is very limited. Covington (1996) tested his algorithm for pairwise phonetic alignment on a small dataset consisting of 82 sequence pairs (henceforth called the Covington Benchmark). Unfortunately, he only presented the results, without providing a gold standard. The test set has nevertheless been used as a benchmark for pairwise phonetic alignment algorithms in a couple of studies (Kondrak 2000, Somers 1999), and the results for the comparison of the algorithms by Covington (1996), Oakes (2000), and Somers (1999), and Kondrak (2000) are given in Kondrak (2002), which makes it very convenient to compare theim with the current proposal.

For multiple phonetic alignments, Prokić et al. (2009) compiled a benchmark database (henceforth called the BulDial Benchmark) consisting of 152 


\begin{tabular}{|l|l|l|l|l|l|l|l|}
\hline Dataset & Languages & PSA & MSA & Words & Taxa & PID & Source \\
\hline \hline Andean & Andean dialects (Aymara, Quechua) & 619 & 76 & 883 & 20 & 55 & SAL \\
\hline Bai & Bai dialects & 889 & 90 & 1416 & 17 & 32 & BDS, Wang 2006a \\
\hline Bulgarian & Bulgarian dialects & 1515 & 152 & 32418 & 197 & 48 & Prokić et al. 2009 \\
\hline Dutch & Dutch dialects & 500 & 50 & 3024 & 62 & 44 & MAND \\
\hline French & French dialects & 712 & 76 & 3810 & 62 & 41 & TPPSR \\
\hline Germanic & Germanic languages and dialects & 1110 & 111 & 4775 & 45 & 32 & LOE \\
\hline Japanese & Japanese dialects & 219 & 26 & 224 & 10 & 40 & Shirō 1973 \\
\hline Norwegian & Norwegian dialects & 501 & 51 & 2183 & 51 & 46 & NORDAVINDEN \\
\hline Ob-Ugrian & Uralic languages & 444 & 48 & 689 & 21 & 45 & GLD \\
\hline Romance & Romance languages & 297 & 30 & 240 & 8 & 37 & LOE \\
\hline Sinitic & Chinese dialects & 200 & 20 & 20 & 40 & 35 & YINKU \\
\hline Slavic & Slavic languages & 120 & 20 & 81 & 5 & 38 & DERKSEN \\
\hline
\end{tabular}

Table 4.8: Data sources of the gold standard for phonetic alignment

manually edited MSAs covering 197 taxa and more than 30000 words taken from Bulgarian dialect data (Buldialect). Prokić et al. (2009) also reported the results of the ALPHAMALIG algorithm for multiple sequence alignment (Alonso et al. 2004) on the BulDial Benchmark. These results were directly compared with those obtained by an early version of SCA (List 2012b). In List (2012c) a new manually edited benchmark dataset was presented. Including the BulDial Benchmark, kindly provided by the authors of Prokić et al. (2009), it consists of 600 MSAs covering six different language families, 435 different taxa, and a total of 45947 words. The extended benchmark database also included a pairwise partition which was directly extracted from the MSAs by taking the 5506 most divergent, unique word pairs.

For this study, an extended version of the previously used benchmark databases was compiled. The new phonetic alignment benchmark consists of manually edited pairwise and multiple alignment analyses. ${ }^{9}$ The MSA partition of the phonetic alignment benchmark consists of 750 manually edited MSAs, covering eight language families, 528 different taxa (language varieties), and 50089 words (14 217 of them unique). The database consists of 12 partitions which either correspond to a language family, or to the dialects of a single language variety. The dataset is further divided into four subsets, covering different regions of diversity, whereas diversity is measured as the average percentage identity of an alignment (see Equation 3.2 on page 74).

9 The full data is available as part of the supporting online material accompanying this study (see Supplementary Material I). 
The subsets and their range are listed in Table 4.9. The PSA benchmark, consisting of 7126 sequence pairs, was extracted automatically by selecting the most divergent sequence pairs from each partition of the benchmark for multiple phonetic alignments, using percentage identity as a measure of divergence. The alignments were edited manually by different contributors. Table 4.8 gives an overview over the different partitions of the data, along with the number of files, words and taxa, the average percentage identity (PID) of the respective partition, and the data sources. In Figure 4.12 the similarity of alignments in the pairwise and the multiple partition of the benchmark is plotted in histograms.

Thompson (2009: 154f) lists four requirements for benchmark databases for computational tasks in biology: (1) relevance, (2) solvability, (3) accessibility, and (4) evolution. Relevance refers to the tests in the benchmark which should be "representative of the problems that the system is reasonably expected to handle in a natural [...] setting" (ibid.: 154). Solvability refers to the tasks presented by the benchmark. They should not be "too difficult for all or most tools" (ibid.: 154f), in order to allow for comparisons between different algorithms and methods. Accessibility refers to both the easiness to obtain and to use the data. Evolution refers to the requirement that benchmarks change constantly in order to avoid that programs are being optimized with respect to the benchmark instead of the general task the benchmark was designed to represent. The current version of the phonetic alignment benchmark was designed to fulfil these requirements as good as possible: The number of datasets from different languages was drastically increased, both in comparison to the Covington Benchmark and the BulDial Benchmark. In order to guarantee solvability, only cognate sets with little morphological variation are included.

\begin{tabular}{|c|c|c|c|}
\hline Subset & Range & MSAs & PID Ø \\
\hline \hline PID_100 & $100-70$ & 15 & 76 \\
\hline PID_70 & $70-50$ & 207 & 57 \\
\hline PID_50 & $50-30$ & 438 & 38 \\
\hline PID_30 & $30-0$ & 90 & 19 \\
\hline
\end{tabular}

Table 4.9: Four subsets of the benchmark for multiple phonetic alignment 


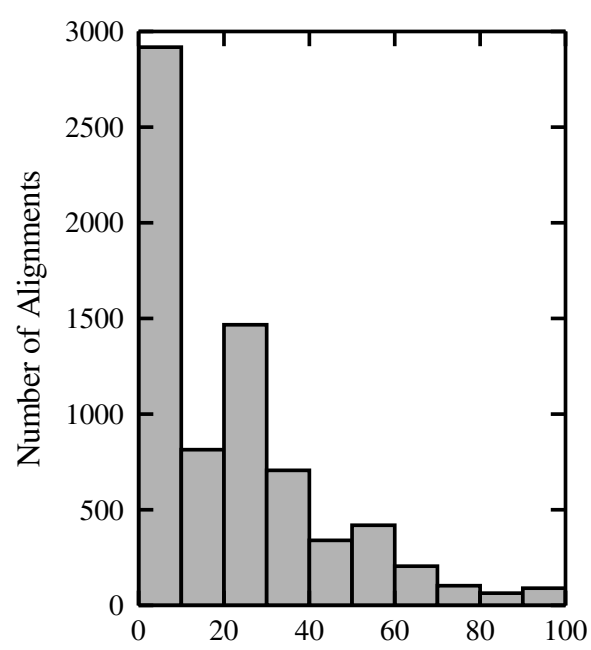

(a) Pairwise (PID: 20)

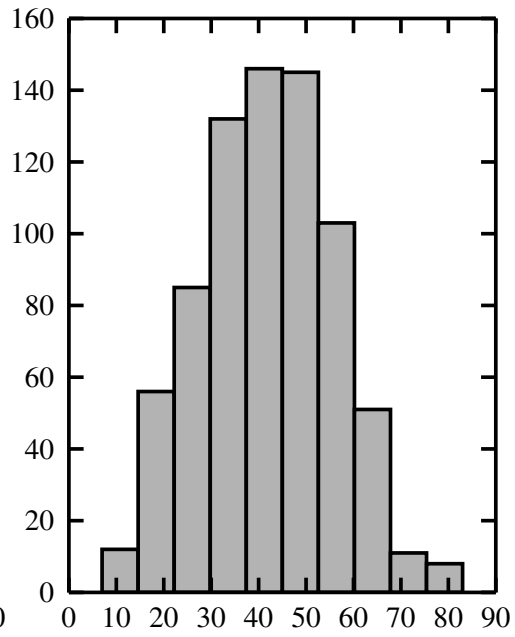

(b) Multiple (PID: 42)

Figure 4.12: Percentage Identity (PID) of the alignments in the gold standard for pairwise (a) and multiple alignments (b).

In order to guarantee consistency and applicability, all words are encoded in IPA. Only the last of the four requirements cannot be addressed at the moment. Since - in contrast to biology - phonetic alignment at the moment still plays a minor role in historical linguistics, it is not clear whether it will be possible to find the resources to change the current benchmark regularly.

\section{Evaluation Measures}

Given a reference set (a gold standard) and a test set (the output of an algorithm), the simplest way to test how well an alignment algorithm performs is to calculate the perfect alignments score (PAS), i.e. the proportion of alignments which are identical in test set and gold standard. Since this score only reveals very strong tendencies, a couple of different methods have been proposed to test how well alignment algorithms perform in comparison with benchmark datasets (Prokić et al. 2009, Thompson et al. 1999). The comparison of alignments produced by automatic methods with alignment benchmarks, however, is a complicated task, and all these methods bear certain shortcomings. 
When comparing two alignments, there are two basic perspectives that can be taken, namely (1) the row perspective, and (2) the column perspective. The former focuses on the rows in both the reference and the test alignment, and the latter focuses on the columns. A simple way to compare a reference with a test alignment is to base the comparison solely on one of the two perspectives. For the row perspective, such a comparison is very straightforward, since both the reference and the test alignment have always the same number of sequences (rows). One may therefore simply calculate a row score (RS) by applying the formula:

$$
R S=100 \cdot \frac{\left|R_{t} \cap R_{r}\right|}{\left|R_{t}\right|},
$$

where $R_{t}$ is the set of rows in the test alignment and $R_{r}$ is the set of rows in the reference alignment. The RS is not used in biological applications. This may be due to the fact that the score may yield some intuitively strange results, especially when only two sequences are aligned. Thus, in case of pairwise alignments, one may encounter the strange situation that one of the rows is identical in test and reference set, while the other row is not. The resulting score of 0.5 seems to be meaningless in such a case, since nothing is said about the degree of difference between test and reference alignment.

For the column perspective, the comparison becomes a little bit more complicated, since the number of columns in test and reference alignment may differ. In applications of information retrieval it is common to evaluate algorithms by calculating their precision and recall. Precision refers to the proportion of items in the test set that also occur in the reference set. Recall refers to the proportion of items in the reference set that also occur in the test set (Witten and Frank 2005: 171). Transferring this to the column perspective, one can define the column precision $(\mathrm{CP})$ as:

$$
C P=100 \cdot \frac{\left|C_{t} \cap C_{r}\right|}{\left|C_{t}\right|},
$$

where $C_{t}$ is the set of columns in the test alignment and $C_{r}$ is the set of columns in the reference alignment. Accordingly, the column recall (CR) may be defined by modifying Equation 4.5 into:

$$
C R=100 \cdot \frac{\left|C_{t} \cap C_{r}\right|}{\left|C_{r}\right|} .
$$




\begin{tabular}{|c|cccc|cccccc|ccccc|}
\hline No. & \multicolumn{1}{|c|}{ Reference } & \multicolumn{1}{|c|}{ Test (columns) } & \multicolumn{1}{|c|}{ Test (rows) } & \\
\hline \multirow{4}{*}{ (a) } & A & B & C & D & A & B & C & - & D & A & B & C & - & D \\
& A & B & - & D & A & B & - & D & - & A & B & - & D & - \\
& A & - & C & - & A & - & C & - & - & A & - & C & - & - \\
\hline \multirow{4}{*}{ (b) } & A & B & C & D & A & B & C & D & & A & B & C & D & \\
& A & B & - & D & A & B & - & D & & & A & B & - & D & \\
& A & - & C & - & - & A & - & C & & & - & A & - & C & \\
\hline
\end{tabular}

Table 4.10: Row and column score bias resulting from small errors in single sequences. Shaded regions indicate rows and columns which differ from the reference alignment.

Since both CP and CR measure different aspects of alignment similarities (Rosenberg and Ogden 2009: 198), it is useful to define a more general score that combines both of them. Following the suggestion of Lassmann and Sonnhammer (2002) and Rosenberg and Ogden (2009: 186), a general column score (CS) can be defined by taking the average of $\left|C_{t}\right|$ and $\left|C_{r}\right|$ (which is equivalent with calculating the harmonic mean of both scores):

$$
C S=100 \cdot \frac{\left|C_{t} \cap C_{r}\right|}{\frac{1}{2} \cdot\left(\left|C_{r}\right|+\left|C_{t}\right|\right)}=100 \cdot 2 \cdot \frac{\left|C_{t} \cap C_{r}\right|}{\left|C_{r}\right|+\left|C_{t}\right|}
$$

In contrast to the RS, the CS is often used in biology (Lassmann and Sonnhammer 2002, Thompson et al. 1999). The definition for the CS, however, varies in the literature. In most approaches, it corresponds to the column precision as it was defined in Equation 4.5, but in practice it seldom differs from the average of column precision and recall. For reasons of consistency and simplicity, I will therefore only use the general CS as defined in Equation 4.7 in this study.

Both measures, the row score and the column score, have specific drawbacks. Since neither of them distinguishes degrees of similarity between columns and rows, errors resulting from small differences in test and reference alignment may yield large differences in the scores. Table 4.10 illustrates this bias, by giving examples where (a) one misaligned sequence results in the difference of all rows, and (b) one misaligned sequence results in the difference of all columns. As a result, the RS of (a) and the CS of (b) is 0 , suggesting that there is no similarity at all between reference and test alignment. Nevertheless, 
both scores are useful to give a rough estimation of alignment accuracy. Being very conservative, they are especially capable to point to large differences and strong tendencies when comparing the performance of several algorithms.

More fine-graded measures for the evaluation of alignment accuracy in biology are based on the comparison of reference and test alignment on a pairwise basis. This can easily be done by comparing the aligned residue pairs (segment pairs) in reference and test alignment. Similar to the calculation of columns scores, the pair precision (PP) can be defined as:

$$
P P=100 \cdot \frac{\left|P_{t} \cap P_{r}\right|}{\left|P_{t}\right|},
$$

where $P_{t}$ is the set of all aligned residue pairs in the test alignment and $P_{r}$ is the set of all aligned residue pairs in the reference alignment. Accordingly, the pair recall (PR) can be defined as:

$$
P R=100 \cdot \frac{\left|P_{t} \cap P_{r}\right|}{\left|P_{r}\right|},
$$

and a general pair score (PS) can be defined by taking the harmonic mean of pair precision and pair recall:

$$
P S=100 \cdot 2 \cdot \frac{\left|P_{t} \cap P_{r}\right|}{\left|P_{r}\right|+\left|P_{t}\right|} .
$$

PR is the most widely used pair score in biology, where it is usually termed sum-of-pairs score (SPS, cf. Thompson et al. 1999). Using only the PR, and not the more general PS, however, has certain drawbacks. Since gaps are ignored, in all pair scores, a situation may arise where pair precision or pair recall yield a score of 1 , indicating full identity of reference and test alignment, although they are in fact different. Thus, the PR (or SPS) for reference and test alignment in Table 4.11a is 1 since all pairs which occur in the reference alignment also occur in the test alignment. That the test alignment itself has two more pairs which do not occur in the reference alignment, is ignored by this measure. The same holds for the PP when comparing reference and test alignment in Table 4.11b. Using the more general PS instead, these problems can be easily avoided. Instead of 1 , the PS for $4.11 \mathrm{a}$ is $100 \cdot 2 \cdot \frac{7}{16}=87.5$ and for $4.11 \mathrm{~b}$ it is $100 \cdot 2 \cdot \frac{4}{11}=72.0$.

Prokić et al. (2009) propose two additional scores as a measure of alignment accuracy: the column dependent evaluation (CDE) and the modified rand in$\operatorname{dex}(\mathrm{MRI})$. The CDE is similar to the CS, but it takes the similarity instead of 


\begin{tabular}{|c|c|c|c|c|c|c|c|c|c|c|c|}
\hline No. & \multicolumn{4}{|c|}{ Reference } & \multicolumn{5}{|c|}{ Test } & Pairs (Reference) & Pairs (Test) \\
\hline \multirow{3}{*}{ (a) } & $\mathrm{A}$ & - & $\mathrm{C}$ & D & A & $\mathrm{C}$ & D & & & $\mathrm{AA},-\mathrm{B}, \mathrm{CC}, \mathrm{DD}$ & $\mathrm{AA}, \mathrm{CC}, \mathrm{DD}$, \\
\hline & A & B & - & D & A & B & D & & & $A A,--, C-, D D$, & $\mathrm{AA}, \mathrm{CB}, \mathrm{DD}$, \\
\hline & A & - & C & - & A & $\mathrm{C}$ & - & & & $\mathrm{AA},-\mathrm{B},-\mathrm{C}, \mathrm{DD}$ & $\mathrm{AA}, \mathrm{BC}, \mathrm{DD}$ \\
\hline \multirow{3}{*}{ (b) } & A & - & $\mathrm{C}$ & D & A & - & $\mathrm{C}$ & D & - & $\mathrm{AA}, \mathrm{B}-, \mathrm{C}-, \mathrm{DD}$, & $\mathrm{AA}, \mathrm{B}-, \mathrm{C}-, \mathrm{D}-,-\mathrm{D}$ \\
\hline & A & B & - & D & A & B & - & - & D & $A A,--, C C, D D$, & $\mathrm{AA},--, \mathrm{CC},--,--$ \\
\hline & A & - & $\mathrm{C}$ & - & A & - & $\mathrm{C}$ & - & - & $\mathrm{AA},-\mathrm{B},-\mathrm{C}, \mathrm{DD}$ & $A A,-B,-C,-D, D-$ \\
\hline
\end{tabular}

Table 4.11: Bias in pair precision (a) and pair recall (b). Pairs shaded in light gray indicate gaps and are ignored when calculating the scores. Pairs shaded in dark gray are ignored in the calculation.

the identity of the columns into account. The MRI is similar to the pair scores, since it ignores the placement of gaps, and compares reference and test alignment from a pairwise perspective. Both the CDE and the MRI generally yield similar tendencies as either the column score or the pair score. For this reason and because of the broad use of column and pair scores in biology, only RS, CS, and PS will be taken as evaluation measures in the following.

\section{Results}

All results which are reported in the following were achieved by using the most recent version the SCA method along with identical parameters for the different parts of the analysis. All parameters correspond to the default settings of the SCA method as they are implemented in LingPy. In all alignment analyses, the GOP was set to -2 . The relative weights for the modification of gap penalties follow the default parameters of LingPy (see Table 4.4). Instead of a fixed gap extension penalty, SCA employs a gap extension scale, by which an extended gap is modified. This value was set to 0.5. Thus, in extended gaps the gap scores were halved by the algorithm. These settings were used in both PPA and MPA analyses. In all MPA analyses, the guide tree was calculated using the Neighbor-Joining algorithm (Saitou and Nei 1987). In consistencybased MPA analyses, the GOP for global alignments was set to -2 , and for local alignments it was set to -1 . All results of the analyses which are discussed in the following are available as part of the supporting online material accompanying this study (see Supplementary Material II). 


\begin{tabular}{|l|l||c|c|c|c|c|c|c|}
\hline \multirow{2}{*}{ Subset } & \multirow{2}{*}{ PSA } & \multicolumn{9}{|c|}{ Score } & \multicolumn{3}{|c|}{ SCA } \\
\cline { 5 - 9 } & & \multirow{2}{*}{ Covington } & \multirow{2}{*}{ Somers } & \multirow{2}{*}{ Oakes } & \multirow{2}{*}{ Kondrak } & & \\
\cline { 5 - 9 } & & & & & & DOLGO & SCA & ASJP \\
\hline \hline Spanish-French & 20 & 19.0 & 17.0 & 15.0 & 20.0 & 18.0 & 20.0 & 20.0 \\
\hline English-German & 20 & 18.0 & 18.0 & 16.0 & 18.5 & 20.0 & 20.0 & 19.0 \\
\hline English-Latin & 25 & 18.1 & 19.5 & 9.0 & 24.0 & 24.0 & 24.0 & 24.0 \\
\hline Fox-Menomini & 10 & 9.0 & 9.0 & 9.0 & 9.5 & 9.0 & 9.0 & 10.0 \\
\hline Other & 7 & 4.7 & 3.0 & 4.0 & 6.0 & 5.0 & 7.0 & 7.0 \\
\hline \hline Total & 82 & 68.8 & 66.5 & 53.0 & 78.0 & 76.0 & 80.0 & 80.0 \\
\hline
\end{tabular}

Table 4.12: Performance of different alignment algorithms on the Covington benchmark.

Pairwise Phonetic Alignment Kondrak (2002) uses the Covington Benchmark in order to compare the performance of the algorithms of Covington (1996) and Somers (1999), and Oakes (2000) with his ALINE algorithm (Kondrak 2000). The evaluation measure is roughly identical with the abovementioned PAS score, i.e. the number of identically aligned sequence pairs in reference and test set is counted. If an algorithm yields more than one output for a given pair, the results are averaged. In order to get a first impression regarding the general quality of SCA alignments in comparison with other methods for pairwise sequence comparison, the Covington Benchmark was analyzed using the three standard models of LingPy (DOLGO, SCA, ASJP) with their respective default parameters and the semi-global extension to the Needleman-Wunsch algorithm. The results for the three models along with the scores achieved by the other algorithms (taken from Kondrak 2002: 64) are listed in Table 4.12. As can be seen from the table, both the SCA and the ASJP model perform slightly better than Kondrak's ALINE, whereas the DOLGO model performs slightly worse, although it still outperforms all the other algorithms. ${ }^{10}$

Although often used for the assessment of alignment quality, the Covington Benchmark is clearly no good benchmark for pairwise sequence alignments. Apart from being too small, both regarding the number of sequence pairs and the number of languages being covered, many of the alignments simply fail to be a real challenge for an alignment algorithm (ibid.: 61). This is also

$\overline{10}$ The items where the three models differ from the gold standard are given in Appendix C.1. 
reflected in the average PID of 37, which is rather high for a pairwise alignment benchmark. The pairwise gold standard of the phonetic alignment benchmark consists of 7126 aligned word pairs with an average PID of 20. It should therefore be much more challenging than the Covington Benchmark.

The PSA gold standard was analyzed using the three different sound-class models provided by LingPy and the traditional Needleman-Wunsch algorithm for global pairwise alignment. In order to test to what degree the new approaches to sequence modelling discussed in Section 4.1 would influence the results of pairwise alignment analyses, four different analyses for each of the three different modes were carried out: (1) a simple global alignment analysis (Basic), (2) a global alignment analysis in which gap costs and substitution scores were scaled in dependence of prosodic environments (Scale), (3) a simple global alignment analysis which was sensitive to secondary sequence structures as extracted from tone markers in South-East Asian languages and word boundaries in the Dutch partition of the pairwise phonetic alignment benchmark (Secondary), and (4) a global alignment analysis which was sensitive to both prosodic environments and secondary sequence structures (Sec-Scale).

\begin{tabular}{|c|c||c|c|c|c|}
\hline \multirow{2}{*}{ Model } & \multicolumn{1}{|c||}{ Measure } & \multicolumn{4}{|c|}{ Mode } \\
\cline { 3 - 6 } & & Basic & Scale & Secondary & Sec-Scale \\
\hline \hline \multirow{4}{*}{ DOLGO } & PAS & 80.52 & 83.23 & 81.74 & 84.87 \\
\cline { 2 - 6 } & RS & 84.47 & 85.86 & 85.39 & 87.01 \\
\cline { 2 - 6 } & CS & 88.31 & 90.55 & 89.26 & 91.31 \\
\cline { 2 - 6 } & PS & 92.70 & 94.21 & 93.33 & 94.70 \\
\hline \hline \multirow{4}{*}{ ASJP } & PAS & 83.64 & 84.62 & 85.00 & 86.09 \\
\cline { 2 - 6 } & RS & 86.48 & 86.82 & 87.49 & 87.89 \\
\cline { 2 - 6 } & CS & 91.00 & 91.63 & 91.88 & 92.47 \\
\cline { 2 - 6 } & PS & 94.53 & 94.97 & 95.09 & 95.50 \\
\hline \hline \multirow{4}{*}{ SCA } & PAS & 84.98 & 86.57 & 86.29 & 88.18 \\
\cline { 2 - 6 } & RS & 87.59 & 88.33 & 88.61 & 89.54 \\
\cline { 2 - 6 } & CS & 91.69 & 92.83 & 92.68 & 93.74 \\
\cline { 2 - 6 } & PS & 94.90 & 95.67 & 95.54 & 96.24 \\
\hline
\end{tabular}

Table 4.13: Results of the PSA Analysis 


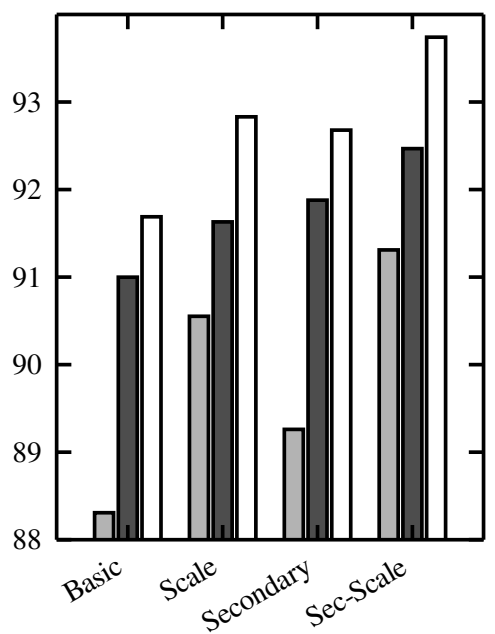

(a) Column score

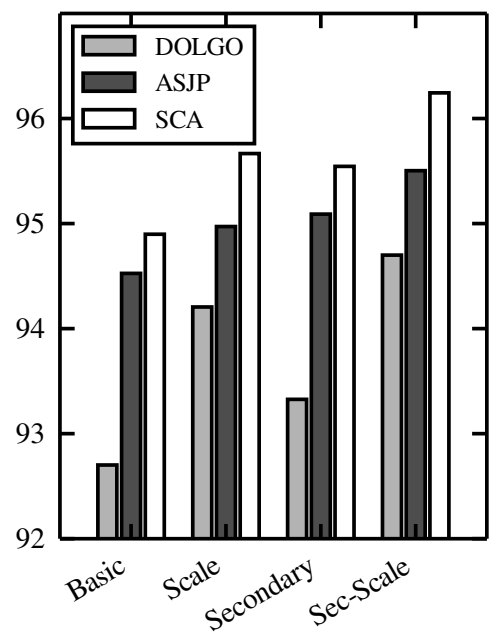

(b) Pair score

Figure 4.13: Results of the PSA analysis

As can be seen from the results shown in Table 4.13 and Figure 4.13, all three complex modes (Scale, Secondary and Sec-Scale) improve the accuracy of the pairwise alignment analyses compared to the Basic mode independent of the evaluation measure. The improvement is significant in all cases with $p<0.01$, using the Wilcoxon signed rank test as suggested by Notredame et al. (2000). The combination of both the Scale and the Secondary mode shows the greatest improvement, yielding the highest scores regardless of measure or model. In all analyses, the SCA model performs best, followed by the ASJP model and the DOLGO model, the differences being significant between all models ( $p<0.01$, using the Wilcoxon test). Apparently, both prosodic context and secondary alignment are very useful extensions for automatic alignment analyses.

Secondary alignment is, of course, only useful, if secondary sequence structures are relevant for an alignment analysis, as it is the case for tone languages. However, of the 7126 alignments in the pairwise benchmark, only 1089 , i.e. $15 \%$, come from South-East-Asian languages like Chinese or Bai. Given the fact that only $15 \%$ of the data apparently account for a significant improve- 


\begin{tabular}{|c|c||c|c|c|c|}
\hline \multirow{2}{*}{ Model } & \multicolumn{1}{|c||}{ Measure } & \multicolumn{4}{|c|}{ Mode } \\
\cline { 3 - 6 } & & Basic & Scale & Secondary & Sec-Scale \\
\hline \hline \multirow{3}{*}{ DOLGO } & PAS & 75.30 & 75.76 & 83.65 & 84.94 \\
\cline { 2 - 6 } & RS & 81.31 & 82.05 & 87.60 & 88.80 \\
\cline { 2 - 6 } & CS & 81.43 & 84.58 & 87.51 & 88.85 \\
\cline { 2 - 6 } & PS & 87.80 & 89.85 & 91.90 & 92.62 \\
\hline \hline \multirow{3}{*}{ ASJP } & PAS & 79.06 & 79.89 & 87.60 & 88.98 \\
\cline { 2 - 6 } & RS & 83.79 & 84.62 & 90.08 & 91.14 \\
\cline { 2 - 6 } & CS & 87.11 & 88.60 & 92.32 & 93.36 \\
\cline { 2 - 6 } & PS & 91.83 & 92.73 & 95.27 & 95.85 \\
\hline \hline \multirow{3}{*}{ SCA } & PAS & 79.43 & 79.71 & 87.88 & 89.44 \\
\cline { 2 - 6 } & RS & 83.43 & 83.98 & 89.94 & 91.23 \\
\cline { 2 - 6 } & CS & 85.19 & 87.76 & 91.33 & 92.81 \\
\cline { 2 - 6 } & PS & 90.52 & 92.22 & 94.64 & 95.50 \\
\hline
\end{tabular}

Table 4.14: Results of the PSA analysis (tone language partition)

ment in alignment accuracy, a further analysis was carried out. This time, only the 1089 alignments belonging to tone languages were analyzed. As can be seen from the results given in Table 4.14 and Figure 4.14, the improvement resulting from the new sequence models becomes even more apparent. Comparing only the PAS, reflecting the number of perfectly aligned word pairs, the Sec-Scale analyses are all between 9 and 10\% better than the Basic analyses in all models. The improvement of the complex modes compared to the Basic mode is again significant in all cases $(p<0.01)$ As in the previous analyses, the DOLGO model performs worse than ASJP and SCA. This time, however, the ASJP model performs better than SCA in all modes.

So far, all tests were based on global alignment analyses only. Hence, as a final part of the evaluation of pairwise sound-class-based phonetic alignments, the performance of the four different alignment modes (global, semi-global, local, and diagonal, see Section 3.3.2) on pairwise benchmark was tested. Since the output of pairwise local alignment analyses differs from the output of the other modes, the evaluation of the local alignments was only based on the core part of the alignments, i.e. the part which remains when all consecutive gaps in the beginning and the end of an alignment are stripped of. 


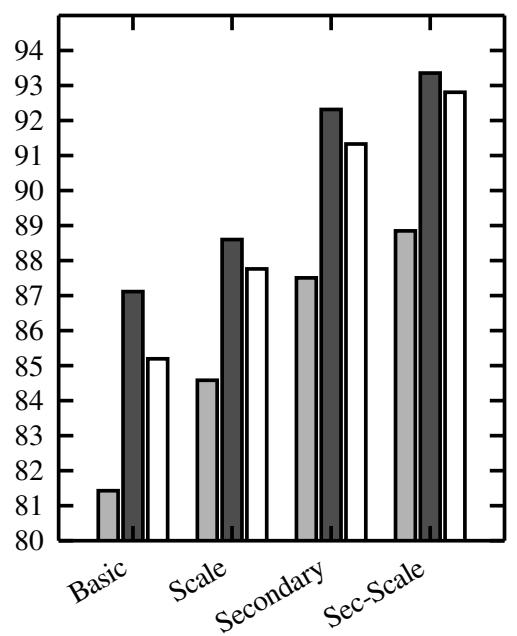

(a) Column score.

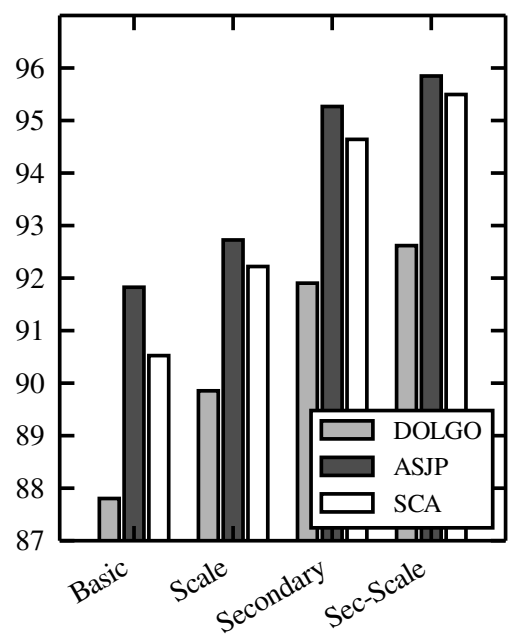

(b) Pair score.

Figure 4.14: Results of the PSA analysis (tone language partition)

For this analysis, only the Sec-Scale mode was used, since it showed the best performance in the previous analyses.

\begin{tabular}{|c|c||c|c|c|c|}
\hline \multirow{2}{*}{ Model } & \multirow{2}{*}{ Measure } & \multicolumn{4}{|c|}{ Mode } \\
\cline { 3 - 6 } & & Global & Semi-Global & Local & Diagonal \\
\hline \hline \multirow{3}{*}{ DOLGO } & PAS & 84.87 & 85.22 & 85.56 & 84.23 \\
\cline { 2 - 6 } & RS & 87.01 & 87.17 & 85.76 & 86.32 \\
\cline { 2 - 6 } & CS & 91.31 & 91.35 & 92.72 & 91.30 \\
\cline { 2 - 6 } & PS & 94.70 & 94.68 & 95.03 & 94.84 \\
\hline \hline \multirow{4}{*}{ ASJP } & PAS & 86.09 & 85.87 & 85.52 & 83.53 \\
\cline { 2 - 6 } & RS & 87.89 & 87.83 & 86.05 & 84.75 \\
\cline { 2 - 6 } & CS & 92.47 & 92.23 & 92.89 & 91.93 \\
\cline { 2 - 6 } & PS & 95.50 & 95.35 & 95.15 & 95.17 \\
\hline \hline \multirow{3}{*}{ SCA } & PAS & 88.18 & 88.25 & 84.51 & 76.76 \\
\cline { 2 - 6 } & RS & 89.54 & 89.42 & 84.87 & 76.70 \\
\cline { 2 - 6 } & CS & 93.74 & 93.61 & 93.78 & 90.18 \\
\cline { 2 - 6 } & PS & 96.24 & 96.12 & 95.79 & 94.39 \\
\hline
\end{tabular}

Table 4.15: Results of the PSA analysis for different alignment modes 


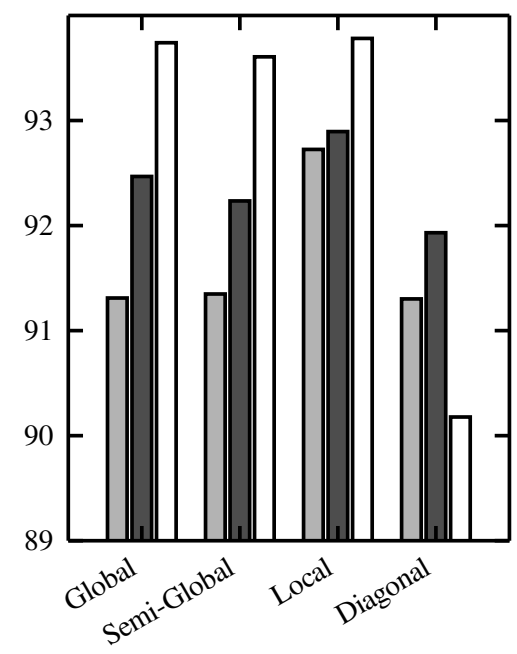

(a) Column score

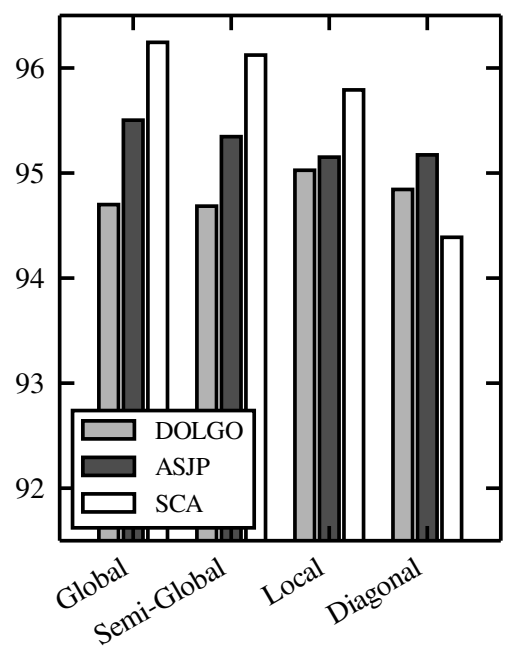

(b) Pair score

Figure 4.15: Results of the PSA analysis for different alignment modes

The results given in Table 4.15 and Figure 4.15 might come as a surprise, since - in contrast to the previous analyses, which all showed a more or less general tendency throughout all sound-class models - the different alignment modes seem to perform quite differently in dependence of the respective soundclass model being used. Thus, for the SCA model, a more or less clear hierarchy ranging from global via semi-global and local to diagonal mode can be determined. ${ }^{11}$ The ASJP model seems to have a similar tendency, yet the differences between the diagonal mode and the other modes are much smaller. For the DOLGO model, the column and pair scores of the Diagonal mode are equal or even better than for the Global and Semi-Global mode, and the best results in terms of pair and column scores are achieved with the Local mode. Hence, while it seems to be the best choice to align in global mode when using the SCA model, it is much more difficult to find the right mode for the ASJP

11 The high column scores in the local modes for all sound-class models are probably due to the fact that only the core blocks of the gold standard alignments were used for the evaluation of alignments produced by the local mode. The general tendency is therefore much more reliably reflected in the row and pair scores. 


\begin{tabular}{|c|c||c|c|c|c|}
\hline \multirow{2}{*}{ Model } & \multicolumn{1}{|c||}{ Measure } & \multicolumn{4}{|c|}{ Mode } \\
\cline { 2 - 6 } & & Basic & Library & Iteration & Lib-Iter \\
\hline \hline \multirow{3}{*}{ DOLGO } & PAS & 70.13 & 73.07 & 74.13 & 75.60 \\
\cline { 2 - 6 } & RS & 81.35 & 81.46 & 83.69 & 83.77 \\
\cline { 2 - 6 } & CS & 85.80 & 87.36 & 87.75 & 88.49 \\
\cline { 2 - 6 } & PS & 97.19 & 97.82 & 97.85 & 98.12 \\
\hline \hline \multirow{3}{*}{ ASJP } & PAS & 74.00 & 76.53 & 78.93 & 79.33 \\
\cline { 2 - 6 } & RS & 82.98 & 84.46 & 86.04 & 87.05 \\
\cline { 2 - 6 } & CS & 88.05 & 89.52 & 90.53 & 90.87 \\
\cline { 2 - 6 } & PS & 97.69 & 98.58 & 98.46 & 98.87 \\
\hline \hline \multirow{3}{*}{ SCA } & PAS & 76.40 & 77.87 & 79.60 & 81.33 \\
\cline { 2 - 6 } & RS & 85.27 & 85.59 & 88.58 & 89.02 \\
\cline { 2 - 6 } & CS & 89.02 & 90.26 & 91.18 & 91.96 \\
\cline { 2 - 6 } & PS & 97.98 & 98.64 & 98.63 & 98.96 \\
\hline
\end{tabular}

Table 4.16: Results of the MSA analysis

and the DOLGO model. Therefore, no final conclusion regarding the reliability of the four alignment modes can be made. When carrying out phonetic alignment analyses, it is important to find the right balance between models and modes.

Multiple Sequence Alignment As for the analysis of the pairwise benchmark, the multiple benchmark was analyzed using the three different soundclass models provided by LingPy. Again, analyses in four different modes were carried out for each model: (1) a progressive alignment analysis (Basic), (2) a progressive alignment analysis with iterative refinement (Iterate), (3) a consistency-based alignment analysis (Library), and (4) a consistency-based alignment analysis in combination with iterative refinement (Lib-Iterate). The iterative refinement analysis was based on the three heuristics described in Section 4.2.3. The library was created from pairwise global, local, and diagonal alignment analyses of all sequence pairs, and the scoring matrix was created from the library as described in Section 4.2.3. Having confirmed that both prosodic context and secondary alignment may significantly improve alignment analyses, the leading question of this analysis was now to clarify 


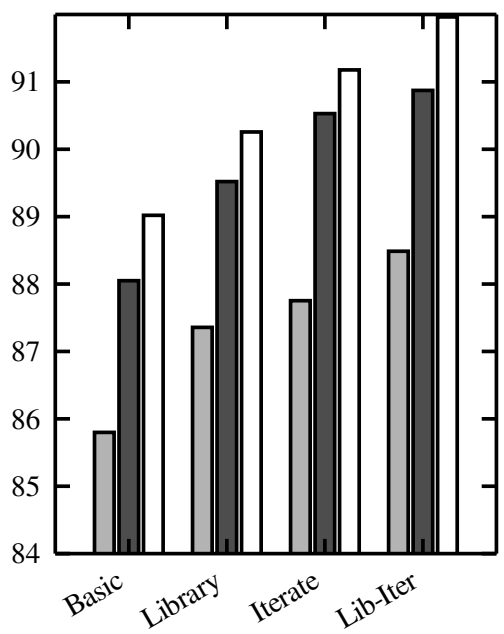

(a) Column score

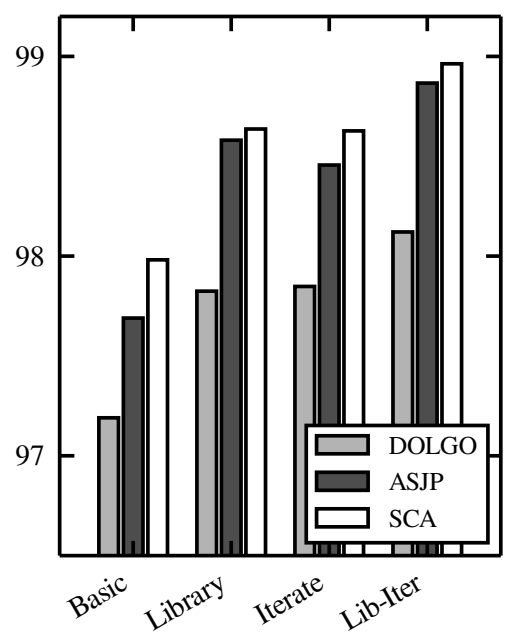

(b) Pair score

Figure 4.16: Results of the MSA analysis

to which degree the modifications to traditional progressive alignment influence the results of multiple alignment analyses.

Looking at the results given in Table 4.16 and Figure 4.16, it becomes obvious that pre- and postprocessing using consistency-based scoring functions and iterative refinement significantly improves the results of multiple alignment analyses ( $p<0.01$ for CS and PS using the Wilcoxon test). The best results are achieved when combining both modes. Similar to the pairwise alignment analyses, the SCA model performs best, followed by ASJP and DOLGO, the difference between all models being significant ( $p<0.01$ for CS and PS).

In order to get some clearer insights into the strengths and weaknesses of the different sound-class models, the performance of the Lib-Iterate analyses on the four subsets of the multiple alignment benchmark was calculated. As can be seen from the results given in Table 4.17 and Figure 4.17, the SCA model again achieves the highest scores in all analyses, followed by ASJP and DOLGO. As one may expect, all models loose accuracy the more divergent the sequences become. The differences between ASJP and SCA, on the one hand, and DOLGO on the other hand, however, are not very great in the PID_70 and 


\begin{tabular}{|c|c||c|c|c|c|}
\hline \multirow{2}{*}{ Model } & \multirow{2}{*}{ Measure } & \multicolumn{4}{|c|}{ Mode } \\
\cline { 2 - 6 } & & PID_100 & PID_70 & PID_50 & PID_30 \\
\hline \hline \multirow{3}{*}{ DOLGO } & PAS & 93.33 & 85.99 & 71.47 & 69.93 \\
\cline { 2 - 6 } & RS & 93.33 & 90.35 & 82.43 & 77.23 \\
\cline { 2 - 6 } & CS & 98.25 & 95.00 & 87.03 & 82.30 \\
\cline { 2 - 6 } & PS & 99.92 & 99.44 & 98.30 & 95.71 \\
\hline \hline \multirow{3}{*}{ ASJP } & PAS & 93.33 & 88.89 & 76.27 & 72.55 \\
\cline { 2 - 6 } & RS & 93.33 & 92.71 & 85.48 & 82.63 \\
\cline { 2 - 6 } & CS & 98.25 & 96.16 & 89.53 & 86.29 \\
\cline { 2 - 6 } & PS & 99.92 & 99.63 & 98.81 & 97.87 \\
\hline \hline \multirow{3}{*}{ SCA } & PAS & 93.33 & 90.34 & 78.40 & 75.16 \\
\cline { 2 - 6 } & RS & 93.33 & 94.56 & 87.72 & 84.26 \\
\cline { 2 - 6 } & CS & 98.25 & 96.78 & 90.66 & 88.03 \\
\cline { 2 - 6 } & PS & 99.92 & 99.74 & 98.88 & 98.01 \\
\hline
\end{tabular}

Table 4.17: Results on different gold standard partitions

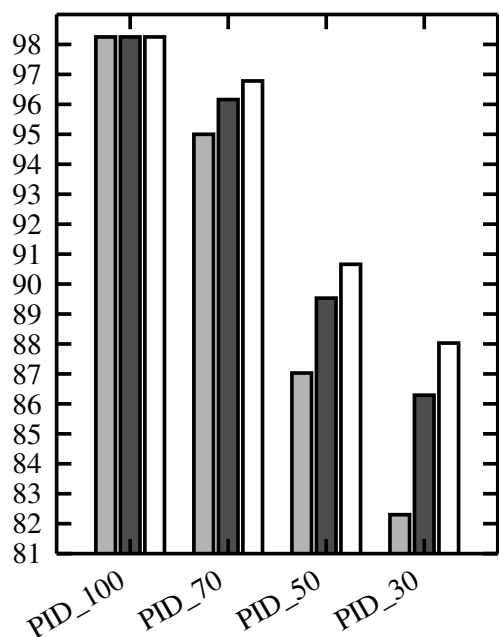

(a) Column score

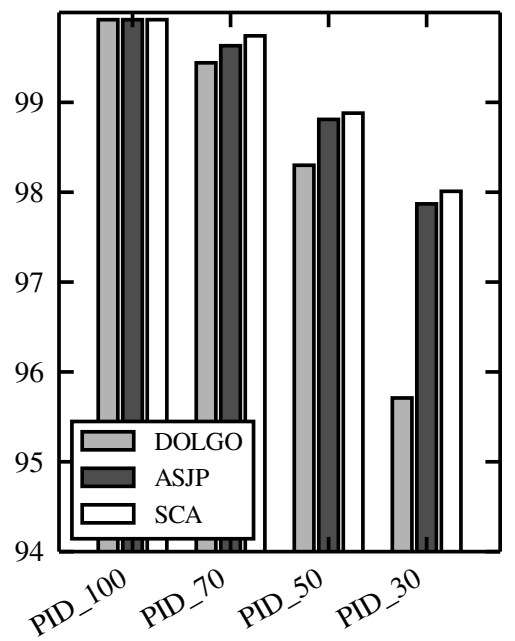

(b) Pair score

Figure 4.17: Results on different gold standard partitions 
the PID_50 subset, while they increase drastically in the PID_30 partition. In List (2012c), a similar increase in the differences could be found between ASJP and SCA. For the most recent version of the SCA approach, this difference can no longer be confirmed. Both model show a comparable accuracy in very divergent sequences. ${ }^{12}$

Swap Detection Of the 750 MSAs in the benchmark, 69 are marked as containing swapped sites. In order to test, how well the method for swap detection described in Section 4.2.3 works, all files were analyzed using the three standard sound-class models of LingPy along with consistency-based scoring functions and iterative refinement (the Lib-Iterate mode). In order to evaluate the accuracy of swap detection, the common distinction between true positives, false positives, and false negatives can be used. True positives, however, can be further divided into those which match the respective columns correctly (true site) and those which do not (wrong site). The results for the analysis are given in Table 4.18. Judging from these results, it is difficult to decide which model really performs best: the DOLGO model with its high recall, or the ASJP model with its high precision. The detection of swapped sites crucially depends on the swap penalty, which was set to -2 for all models. Lowering this penalty will increase the recall, yet it will also decrease the precision, and more testing is needed to find the right balance between false positives and false negatives.

\begin{tabular}{|l|c||c|c|c|}
\hline \multicolumn{2}{|c|}{ Category } & DOLGO & ASJP & SCA \\
\hline \hline \multirow{2}{*}{ True Positive } & True Site & 58 & 51 & 51 \\
\cline { 2 - 5 } & Wrong Site & 2 & 2 & 1 \\
\hline \hline False Positive & 7 & 2 & 2 \\
\hline False Negative & 9 & 16 & 17 \\
\hline
\end{tabular}

Table 4.18: Results of the swap-identification task

\footnotetext{
12 This may be due to the fact that in the new analyses the weights for prosodic context were further refined, resulting in a generally enhanced performance of phonetic alignment analyses using the ASJP model.
} 


\subsubsection{Examples}

Pairwise Phonetic Alignment In Figure 4.18 two different alignment analyses of dialect variants of Dutch berg [berx] 'mountain' are given. The first one is the typical output of a traditional alignment analysis with an extended scoring scheme (SCA-Basic). The second one is based on the same scoring scheme extended by prosodic weights (SCA-Scale). While the Basic analysis wrongly matches the phonetically similar sounds $[\gamma]$ and $[\mathrm{x}]$, the Scale analysis correctly matches $[\gamma]$ with the less similar $[\mathrm{R}]$. The reason can be found in the modified gap penalties and substitution scores of the Scale analysis. While the Basic analysis treats all positions identically, the Scale analysis assigns a lower gap penalty to the insertion of gaps in the end of a word, i.e. [x] in [be:Rex] can be more easily gapped than [R]. Furthermore, the Scale analysis gives increased substitution scores for segments appearing in the same sonority context, such as $[\mathrm{\gamma}]$ and $[\mathrm{R}]$ which both appear in a position of ascending sonority. This also forces the algorithm to prefer the matching of $[\gamma]$ with $[\mathrm{R}]$ over the matching of $[\mathrm{\gamma}]$ with $[\mathrm{x}]$. This is but one example, how positionspecific weights can enhance traditional pairwise alignment analyses.

In the tests on the tone language partition of the benchmark for pairwise alignment analyses, it became especially evident how drastically secondary alignment analyses may increase the accuracy of global alignment analyses. A prototypical example for the difference between primary and secondary alignment analyses is given in Figure 4.19 where two cognates of Chinese

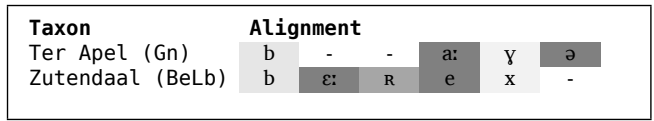

(a) Basic analysis

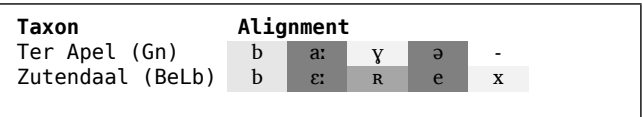

(b) Scale analysis

Figure 4.18: The alignment of dialect variants of Dutch berg 'mountain' in the Basic (a) and the Scale analysis (b). The data is taken from MAND. 
ritóu 日頭 $\left[\mathrm{z}^{5}{ }^{51} \mathrm{t}^{\mathrm{h}} \mathrm{Ou}^{35}\right]$ 'sun' are aligned. The primary analysis wrongly mat-

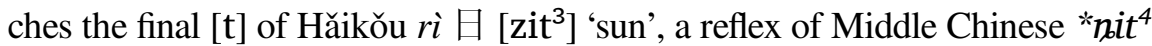
日 'sun', with the initial of Yínchuān tó $u$ 頭 [ $\mathrm{t}^{\mathrm{h}} \mathrm{\partial u}$ ] 'head', a reflex of Middle Chinese *duw ${ }^{1}$ 頭 'head', thereby aligning elements from one syllable in one word with elements from two syllables in the other. Since such a behaviour is prohibited in secondary alignment, the secondary mode correctly matches the corresponding elements of both words with each other.

Analyses using the diagonal alignment mode improved when the DOLGO sound-class model was used, but became worse when using the other models. One reason for this behaviour can be found in the very nature of diagonal alignment. In contrast to global and local alignment, diagonal alignment is based on a very specific scoring function which seeks to maximize the overall alignment score by finding the longest and highest-scoring diagonals (ungapped subalignments). As a result, the method is very conservative regarding diverse sequences and matches only those sounds whose similarity (as assigned by the scoring matrix) is greater than 0 . As a result, many divergent matches that global or local algorithms tolerate for the sake of a maximized global score are not accepted in diagonal alignments. In the SCA model and the ASJP model, this leads to a decrease in alignment accuracy, since the models are considerably fine-graded. The DOLGO model, on the other hand, is very broad, in so far as many phonetically quite different sounds are lumped together. As a result, the conservative behaviour of diagonal alignment is weakened by the sound-class model. Although the conservatism seems to be a disadvantage

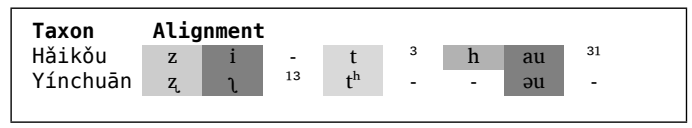

(a) Primary analysis

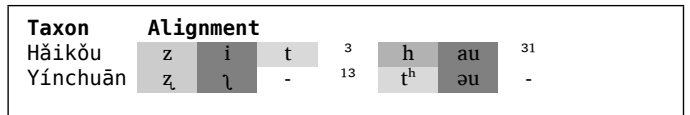

(b) Secondary analysis

Figure 4.19: The alignment of dialect variants of Chinese rìtó 'sun' in the Primary (a) and the Secondary analysis (b). The data is taken from YINKU. 


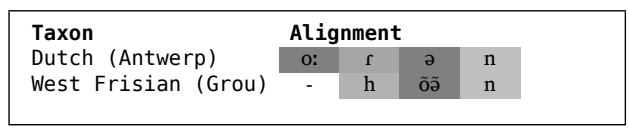

(a) Global mode

\begin{tabular}{|c|c|c|c|c|c|}
\hline Taxon & \multicolumn{5}{|c|}{ Alignment } \\
\hline Dutch (Antwerp) & - & o: & f & $\partial$ & $\mathrm{n}$ \\
\hline West Frisian (Grou) & h & õ & - & - & $\mathrm{n}$ \\
\hline
\end{tabular}

(b) Diagonal mode

Figure 4.20: The alignment of reflexes of Proto-Germanic "xurnan 'horn' in Dutch and West Frisian dialects in the Global (a) and the Diagonal mode (b). The data is taken from $L O E$.

in general, it may turn out to be an advantage in very specific cases. As an example, consider the alignment of Dutch and West Frisian reflexes of ProtoGermanic *hurna- "horn' (KROONEN: 259, based on the SCA model. While the global analysis "accepts" the incorrect matching of [r] and [h], even despite the fact that both occur in different prosodic environments, the diagonal analysis rejects it, correctly aligning only the phonetically most similar segments. The conservative character of diagonal alignments is especially helpful in multiple consistency-based alignments, where diagonal alignments can be used to fill the primary library as a corrective for the less conservative global and local alignment analyses.

Multiple Sequence Alignment The first alignment in Figure 4.21 (MSA 125 in the multiple alignment benchmark) is the typical output of a traditional matrix-based progressive alignment algorithm on a rather tough test alignment, taken from the Bai partition of the phonetic alignment benchmark. Apparently, the phonetic sequences are very diverse, and it is difficult to detect strong similarities when dealing with such an alignment. This is a typical output of traditional matrix-based progressive alignment algorithms. If global similarities cannot be detected, the resulting alignments are mere collections of sound segments in a matrix that are seemingly unreasonably lumped together.

The second alignment in Figure 4.21, on the other hand, is the output of the new consistency-based algorithm that was further improved by iterative 


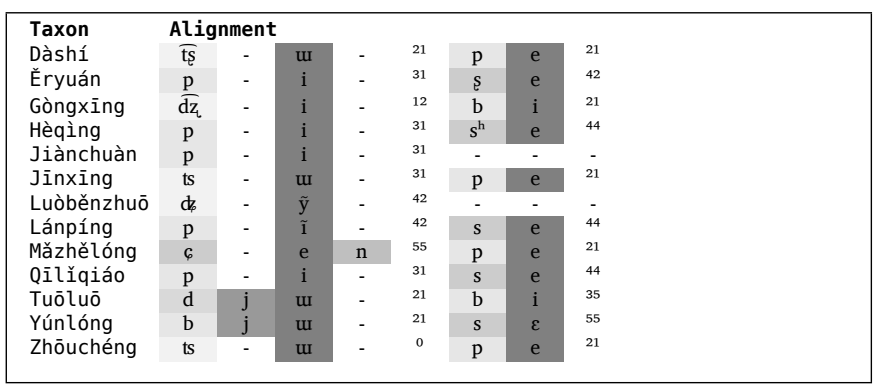

(a) Matrix-based analysis

\begin{tabular}{|c|c|c|c|c|c|c|c|c|c|c|c|c|}
\hline Taxon & Alic & ne & & & & & & & & & & \\
\hline Dàshí & $\overline{\mathrm{t} s}$ & - & $\mathrm{u}$ & - & 21 & $\mathrm{p}$ & - & e & 21 & - & - & - \\
\hline Ěryuán & - & - & - & - & - & $\mathrm{p}$ & - & $\mathrm{i}$ & 31 & s & e & 42 \\
\hline Gòngxīng & $\overline{\mathrm{dz}}$ & - & i & . & 12 & b & - & $\mathrm{i}$ & 21 & - & - & - \\
\hline Hèqìng & & - & - & . & - & $\mathrm{p}$ & - & $\mathrm{i}$ & 31 & $\mathrm{~s}^{\mathrm{h}}$ & e & 44 \\
\hline Jiànchuàn & - & - & - & . & - & $\mathrm{p}$ & - & $\mathrm{i}$ & 31 & - & - & - \\
\hline Jīnxīng & ts & - & u & . & 31 & $\mathrm{p}$ & - & $\mathrm{e}$ & 21 & - & - & - \\
\hline Lánpíng & - & - & - & - & - & $\mathrm{p}$ & - & $\tilde{1}$ & 42 & s & e & 44 \\
\hline Luòběnzhuō & d & - & $\tilde{y}$ & . & 42 & - & - & - & - & - & - & - \\
\hline Mǎzhělóng & 6 & - & e & $\mathrm{n}$ & 55 & $\mathrm{p}$ & - & e & 21 & - & - & - \\
\hline Qīlǐqiáo & - & - & - & - & - & $\mathrm{p}$ & - & $\mathrm{i}$ & 31 & $\mathrm{~s}$ & e & 44 \\
\hline Tuōluō & d & $\mathrm{j}$ & $\mathrm{wI}$ & . & 21 & b & - & $\mathrm{i}$ & 35 & - & - & - \\
\hline Yúnlóng & - & - & - & . & - & b & $\mathrm{j}$ & ш & 21 & $\mathrm{~s}$ & $\varepsilon$ & 55 \\
\hline Zhōuchéng & ts & - & $\mathrm{u}$ & - & 0 & $\mathrm{p}$ & - & e & 21 & - & - & - \\
\hline
\end{tabular}

(b) Consistency-based analysis

Figure 4.21: Alignment of Bai dialect words for 'bark' and 'rain cape' in matrix- (a) and consistency-based alignment analyses (b).

refinement. The method correctly detects the specific local similarities between the words: All words (with the exception of Luòběnzhuō [d\&y $\left.{ }^{42}\right]$ 'rain cape', which might not even be cognate with the other words) have a reflex of Proto-Bai *be ${ }^{1}$ 'skin' (Wang 2006a), probably cognate with Chinese $p \check{\imath}$ 皮 $\left[\mathrm{p}^{\mathrm{h}} \mathrm{i}^{214}\right]$ 'skin' ( $<$ Middle Chinese ${ }^{*} b j e^{1}$ 皮 $<$ Old Chinese *m-paj 皮, OCBS). Apart from this common morpheme, the dialects can be divided into two classes which actually correspond to two different compounds which are only partially cognate. The first class shows a compound of reflexes of ProtoBai *dru ${ }^{3}$ 'tree' with reflexes of the aforementioned Proto-Bai * $b e^{1}$ 'skin', as in Jīnxīng [tsu' ${ }^{31} \mathrm{pe}^{21}$ ], with the meaning 'bark' < 'tree' + 'skin' (Wang 2006a). The second class shows a compound of reflexes of Proto-Bai *be ${ }^{1}$ 
'skin' with reflexes of Proto-Bai ${ }^{h} s^{h} \mathcal{E}^{4}$ 'cloth' (ibid.), as in Ěryuán $\left[\mathrm{pi}^{31} \mathrm{Se}^{42}\right]$, with the meaning 'rain cape' < 'cape made from (palm) bark ( $<$ skin)', (BDS).

Both classes are correctly detected by the algorithm, and the different reflexes are separately aligned. As can be seen from the example, consistencybased methods do not only enhance multiple sequence alignment in biology, but also in linguistics. The specific strength of consistency-based algorithms to be sensitive to both global and local similarities between sequences becomes very apparent in the alignments yielded by the new enhancements of the SCA method.

Swap Detection In order to correctly detect swapped sites in an alignment, it is of great importance that the previous alignment analysis allows the swap to be detected. In Figure 4.22 this is illustrated by contrasting two different alignments (MSA 673 in the multiple alignment benchmark) for reflexes of Proto-Slavic *žbltъ 'yellow' in Bulgarian, Czech, Polish, and Russian (DERKSEN: 565f). The first alignment is based on the ASJP model, using the traditional progressive alignment (Basic mode), the second one is based on the same model but it uses consistency-based progressive alignment with iterative refinement (Lib-Iter). In the output of the Basic mode, the [w] in Polish is misaligned. As a result, no complementary structures can be detected in the alignment, and the method for swap detection fails. In the Lib-Iter mode, the $[\mathrm{w}]$ in Polish is correctly aligned with the laterals in the other languages, and the method for swap detection easily identifies the columns 2, 3, and 4 as a swapped site. In the output, this is marked by the white font color of the sounds which appear in swapped columns.

\subsection{Automatic Cognate Detection}

So far this study focused on alignment analyses as a specific way to compare sequences. In Section 4.1, new approaches to sequence modelling were introduced, and these new sequence models were then used to derive the new method for phonetic alignment presented in Section 4.2. While automatic alignment analyses may surely be useful as a stand-alone tool in historical linguistic and dialectological endeavors, they do not constitute an end in itself. Instead, they are one of the basic tools that can help us approaching one of the "holy grails" of quantitative historical linguistic: the task of automatic cognate detection (ACD). Given the basic practice of historical linguistics to base lan- 


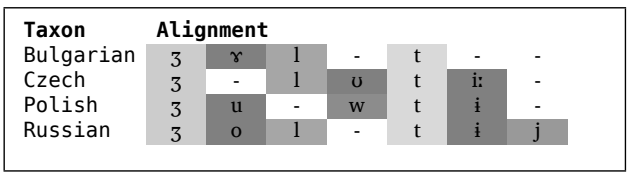

(a) Missed swap (ASJP-Basic)

1

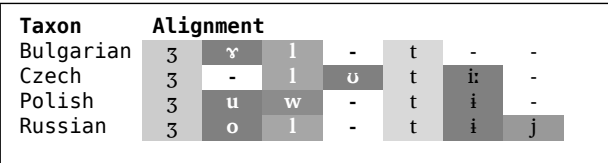

(b) Detected swap (ASJP-Lib-Iter)

Figure 4.22: Missed (a) and detected swaps (b) in the alignment of descendant words of Proto-Slavic *žbltъ 'yellow'.

guage comparison on lexical comparison we can state the cognate detection problem as follows:

Cognate Detection Problem: Given a multilingual word list covering a set of arbitrarily selected languages, find all words that are cognate.

In historical linguistics, this problem is usually approached within the framework of the comparative method, as it was outlined in Section 2.6. The most important aspects of the traditional method for cognate detection are the language-specific notion of word similarity, derived from previously identified regular sound correspondences, and the iterative character of the method, by which proposed list of cognates and sound correspondences are constantly refined and updated. Note that the general cognate detection problem was stated as being indifferent regarding a couple of parameters. It is not specified, how many languages are being compared, or whether the genetical relatedness between these languages should be already proven. It is also left open whether the cognate sets to be identified should be restricted to semantically similar words or whether words that greatly diverged semantically should also be included. Furthermore, the size of the word lists is not specified. For the comparative method, only the last point matters. The method is indifferent regarding the number of languages being compared, it has its own procedure to determine genetical relatedness between languages, and semantically dif- 
ferent but formally similar words have seldom posed a problem for historical linguists. The size of the word lists, however, is of crucial importance for the method, although nobody has so far been able to determine how many items a word lists should at least contain in order to be applicable. That the popular Swadesh-200 word lists (see Section 2.1.5) are surely not enough when questions of remote relationship have to be solved can be easily demonstrated when considering the amount of cognate words in these word lists for some genetically related languages such as as Armenian, English, French, and German: Given that there are maximally 20 cognates between Armenian and the other three languages, it is hardly possible that these cognates are enough to set up a satisfying set of sound correspondences between these languages.

There is a further aspect of the general cognate detection problem that cannot be solved by the comparative method in its strict form: the distinction between common descent due to borrowing and common descent due to inheritance. As it has been discussed earlier in Sections 2.4.1 and 2.5.2, regular sound correspondences do not necessarily point to common descent but may also result from lexical borrowing. The comparative method thus actually doesn't solve the general cognate detection problem, but rather (merely) solves the homologue detection problem:

Homologue Detection Problem: Given a multilingual word list covering a set of arbitrarily selected languages, find all words that are etymologically related.

So far, both the problem of cognate detection and the problem of homologue detection have been stated in general terms. No further preprocessing of the

\begin{tabular}{|c||c|c|c|c|}
\hline & Albanian & English & French & German \\
\hline \hline Albanian & & 0.07 & 0.10 & 0.10 \\
\hline English & 14 & & 0.23 & 0.56 \\
\hline French & 20 & 46 & & 0.23 \\
\hline German & 20 & 111 & 46 & \\
\hline
\end{tabular}

Table 4.19: Number and proportion of cognates within Swadesh-200 word lists of four Indo-European languages. The lower triangle of the table indicates the concrete number of shared cognates. The upper triangle gives the percentage of shared cognates. The cognate counts are based on the data given in Kessler (2001). 
data is required by algorithms or methods that solve such a problem, and no restrictions regarding the character of cognates or etymologically related words are being made. Most algorithms for automatic cognate detection which have been proposed so far do not directly deal with this general homologue detection problem, but rather state the problem more specifically. Among the most common specifications of the problem, is to require that etymologically related words should have the same meaning. This problem can be called the specific cognate detection problem:

Specific Homologue Detection Problem: Given a multilingual word list, covering a set of arbitrarily selected languages, find all words that are etymologically related (homologous) and have the same meaning.

While semantic identity has never been a requirement for the determination of cognacy in historical linguistics, and it is often explicitly stated, that cognacy can often be determined for semantically quite different words (Szemerényi 1970: 15f), it reflects the heuristic practice of many historical linguists to start the search for cognates by comparing semantically similar words, and to proceed to the comparison of semantically more distant words in later stages. ${ }^{13}$ It therefore seems useful to start with the specific homologue detection problem when developing automatic approaches.

\subsubsection{Previous Work}

Although up to today quite a few methods for automatic cognate detection have been proposed, there are only four recent approaches known to me which explicitly deal with the specific homologue detection problem, as it was defined above. The other methods either restrict cognate detection to pairwise word lists (Mackay and Kondrak 2005), or they require specific parameters, such as a guide tree of the languages, to be known in advance (Hall and Klein 2010). Bergsma and Kondrak (2007) first calculate the longest common subsequence ratio between all word pairs in the input data and then use an integer linear programming approach to cluster the words into cognate sets. Unfortunately, their method is only tested on a dataset containing alphabetic transcriptions; hence, no direct comparison with methods that require phonetic transcriptions as input data is possible. Turchin et al. (2010) determine cognacy in multilingual

13 This practice is already reported in Gabelentz (1891: 177-179), who presents his own "basic vocabulary lists", which he calls "Collectaneen zum Sprachvergleiche", as a heuristic tool for the initial stages of language comparison. 
word lists with help of a simple matching criterion: whenever the first two consonants of two words are identical regarding their Dolgopolsky sound-class, the words are judged to be cognate. Hauer and Kondrak (2011) combine specific language-pair features with common metrics for word similarity (longest common subsequence, etc.) to cluster semantically aligned words into cognate sets. Their method doesn"t employ phonetic similarities, since the authors want to keep it applicable to word lists in orthographical encoding. Steiner et al. (2011) propose an iterative approach which starts by clustering words into tentative cognate sets based on their alignment scores. These preliminary results are then refined by filtering words according to similar meanings, computing multiple alignments, and determining recurrent sound correspondences. The authors test their method on two large datasets. Since no gold standard for their test set is available, they only report intermediate results, and their method cannot be directly compared with other methods.

\subsubsection{LexStat - Multilingual Cognate Detection}

The LexStat method for automatic cognate detection in multilingual word lists was first proposed in List (2012a). In the following, the most recent version of the method will be introduced in detail. LexStat combines the most important aspects of the comparative method with recent approaches to sequence comparison in historical linguistics and evolutionary biology. The method employs automatically extracted language-specific scoring schemes and computes distance scores from pairwise alignments of the input data. These languagespecific scoring schemes come close to the notion of regular sound correspondences in traditional historical linguistics. Similar to the SCA method for phonetic alignment presented in Section 4.2, LexStat is also implemented as a part of the LingPy library. It can either be used in Python scripts or directly be called from the Python prompt.

The basic working procedure of LexStat consists of five stages: (1) sequence conversion, (2) preprocessing, (3) scoring-scheme creation, (4) distance calculation, and (5) sequence clustering. In stage (1), the input sequences are converted into tuples consisting of sound classes and prosodic strings. In stage (2), a simple language-independent ACD method is used to derive preliminary cognate sets. In stage (3), a Monte-Carlo permutation test is used to create language-specific log-odds scoring schemes for all language pairs. In stage (4) the pairwise distances between all word pairs, based on the language-specific scoring schemes, are computed. In stage (5), the sequences are clustered into 


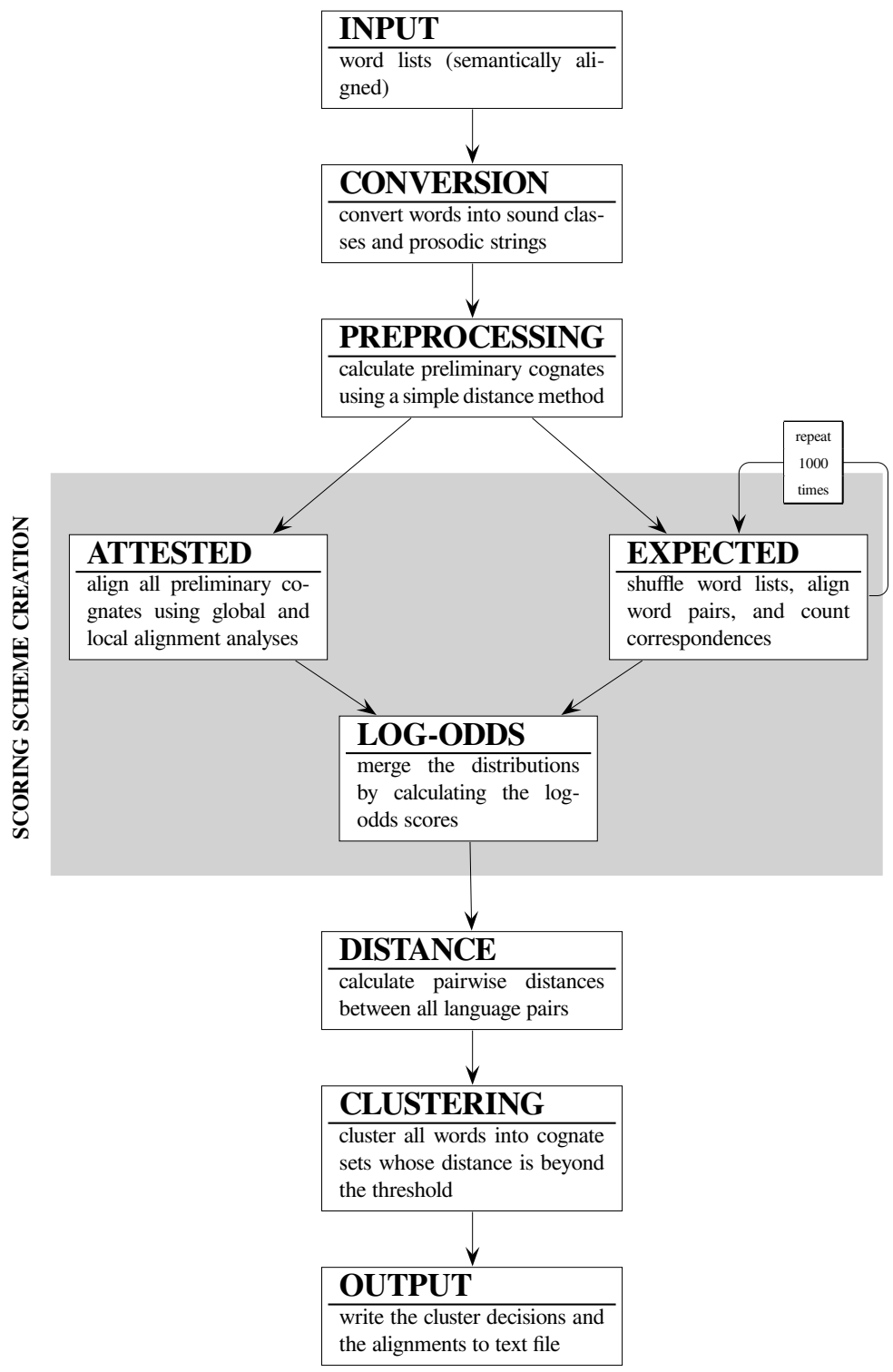

Figure 4.23: Working procedure of the LexStat method for automatic cognate detection. See the text for a detailed description. 
cognate sets whose average distance is beyond a certain threshold. Additionally to these five stages, all cognate sets detected by the method are aligned, using the SCA method for multiple phonetic alignment described in Section 4.2. The aligned output is not directly related to the ACD problem, but merely serves as a tool to display the decisions made by the algorithm in a visually appealing way. The different stages of the working procedure of LexStat are illustrated in Figure 4.23.

\subsubsection{Specific Features}

\section{Input and Output}

The basic input format of LexStat is a tab-delimited text file in which the first line indicates the values of the columns and all words are listed in a separate line. The format is pretty flexible. No specific order of columns or rows is required. Whatever additional data the user wants to include can be included, as long as it is in a separate column. Each word has to be characterized by at least four values given in separate columns: (1) ID, an integer that is used to uniquely identify the word during the calculation, (2) Taxa, the name of the language in which the word occurs, (3) GlossID, an identifier which indicates the meaning of the word and which is used to align the words semantically, and (4) IPA, the phonetic transcription of the word given in IPA. The output format is essentially the same as the input format with one additional cognate ID column (CogID) that indicates the clustering decision of the method: All words that are given the same CogID in the output have been identified as cognates by the method (see Table 4.20). ${ }^{14}$

In addition to the simple output, LexStat offers also the possibility to output the data in aligned form. These multiple alignment analyses that are carried out in the last stage of the LexStat method are no direct requirement for an ACD algorithm, since the main task, the identification of cognate sets, is already accomplished after the clustering stage. However, the specific MSA output format provided by LexStat is very convenient for manual checking and editing of automatically detected cognate sets. If the method is used to aid comparative linguists working on less well-studied language families, it may come in handy to have an aligned representation of all cognate sets proposed by the algorithm. Furthermore, since the language-dependent log-odds

${ }^{14}$ I am very thankful to Steven Moran for pointing me to the advantages of such a flexible input structure. 
scores are also used for the calculation of the alignments, weak positions in the cognate sets will be left unaligned by the algorithm, which may turn out to be a big help when refining ACD analyses manually. Table 4.21 illustrates the basic idea of the aligned output, taking the translations of the basic concept 'woman' in six Germanic languages as an example.

\section{Sequence Conversion}

In the stage of sequence conversion, all input sequences are converted to tuples consisting of sound classes and prosodic strings. Thus, German Sand [zant] 'sand' has the sound-class representation "SANT" and the prosodic string "\#VC\$>", and is internally represented as [ ('S', '\#'), ('A', ' $\left.\mathrm{V}^{\prime}\right),\left(\mathrm{N}^{\prime}, \mathrm{C}^{\prime}\right),\left(\mathrm{C}^{\prime}, \mathrm{I}^{\prime}\right)$ ], and German hei $\beta$ [hars] 'hot' is internally represented as $\left[\left(\mathrm{C}^{\prime}, \mathrm{I}^{\prime} \mathrm{H}^{\prime}\right),\left(\mathrm{I}^{\prime}, \mathrm{I}^{\prime} \mathrm{V}^{\prime}\right), \mathrm{('S}^{\prime}\right.$,

\begin{tabular}{|lllllll|}
\hline ID & Taxa & Word & Gloss & GlossID & IPA & $\ldots$ \\
$\ldots$ & $\ldots$ & $\ldots$ & $\ldots$ & $\ldots$ & $\ldots$ & $\ldots$ \\
21 & German & Erau & woman & 20 & frau & $\ldots$ \\
22 & Dutch & vrouw & woman & 20 & vrau & $\ldots$ \\
23 & English & woman & woman & 20 & womən & $\ldots$ \\
24 & Danish & kvinde & woman & 20 & kvenə & $\ldots$ \\
25 & Swedish & kvinna & woman & 20 & kvi:na & $\ldots$ \\
26 & Norwegian & kvine & woman & 20 & kvinə & $\ldots$ \\
$\ldots$ & $\ldots$ & $\ldots$ & $\ldots$ & $\ldots$ & $\ldots$ & $\ldots$ \\
\hline
\end{tabular}

(a) Input

\begin{tabular}{|lllllll|}
\hline ID & Taxa & Word & Gloss & GlossID & IPA & CogID \\
$\ldots$ & $\ldots$ & $\ldots$ & $\ldots$ & $\ldots$ & $\ldots$ & $\ldots$ \\
21 & German & Frau & woman & 20 & frau & 1 \\
22 & Dutch & vrouw & woman & 20 & vrau & 1 \\
23 & English & woman & woman & 20 & womən & 2 \\
24 & Danish & kvinde & woman & 20 & kvenə & 3 \\
25 & Swedish & kvinna & woman & 20 & kvi:na & 3 \\
26 & Norwegian & kvine & woman & 20 & kvinə & 3 \\
$\ldots$ & $\ldots$ & $\ldots$ & $\ldots$ & $\ldots$ & $\ldots$ & $\ldots$ \\
\hline
\end{tabular}

(b) Output

Table 4.20: Input (a) and output format (b) of LexStat. Four columns are required in the input: ID, Taxa, GlossID, and IPA. An additional column is added in the output. Each word is assigned a specific cognate ID (CogID). All words that have the same CogID have been identified as cognates by the algorithm. 


\begin{tabular}{|c|l|l|l|lllll|}
\hline ID & Language & Word & Gloss & \multicolumn{4}{|l|}{ Alignments } \\
\hline \hline 1 & German & Frau & 'woman' & $\mathrm{f}$ & $\mathrm{r}$ & $\mathrm{au}$ & & \\
1 & Dutch & vrouw & 'woman' & $\mathrm{v}$ & $\mathrm{r}$ & $\mathrm{au}$ & & \\
\hline \hline 2 & English & woman & 'woman' & womən & & & \\
\hline \hline 3 & Danish & kvinde & 'woman' & $\mathrm{k}$ & $\mathrm{V}$ & $\mathrm{e}$ & $\mathrm{n}$ & ə \\
3 & Swedish & kvinna & 'woman' & $\mathrm{k}$ & $\mathrm{v}$ & $\mathrm{i}$ & $\mathrm{n}$ & $\mathrm{a}$ \\
3 & Norwegian & kvine & 'woman' & $\mathrm{k}$ & $\mathrm{v}$ & $\mathrm{i}$ & $\mathrm{n}$ & ə \\
\hline
\end{tabular}

Table 4.21: Aligned output of three cognate sets identified by the LexStat method.

'\$' ) ]. ${ }^{15}$ Identical segments in one language which differ regarding their position in the word are now treated as different segments. As a result, the initial of Sand and the final of hei $\beta$, which are represented by identical sound classes, are still kept separate, because their tuple representations differ ( ( ' $S$ ' ' ' \# ' ) vs. ( ' $S$ ', ' $\$$ ' ) ). This representation constitutes a novel approach to model phonetic context. It has the advantage of being more "abstract" than bi- or $n$ gram approaches which are usually used to incorporate context sensitivity in alignment analyses (Heeringa et al. 2006).

\section{Scoring-Scheme Creation}

The idea to compute language-specific similarities by trying to detect regular sound correspondences automatically is definitely not new, and there are many different methods described in the literature (Guy 1994, Kondrak 2003, Oakes 2000, Starostin 2008a). Unfortunately, the applicability and availability of most of these approaches is rather limited. Some are only described in the literature (Guy 1994, Starostin 2008a), some cover only a limited range of sounds (Oakes 2000), and some require specific codings instead of the general IPA standard (Kondrak 2003). The general strategy that all approaches usually follow is to create an attested distribution by calculating the (possible) links (segment correspondences, residue pairs in biology) between all word pairs in a bilingual word list, and to compare this distribution with an expected distribution, i.e. with a distribution of residue pairs one would get if the lan-

${ }^{15}$ In this description of the LexStat method, I generally use the SCA model for the examples. 
guages were not related. This idea is similar to the way the BLOSUM substitution matrices are calculated in evolutionary biology (see Section 3.3.3). The main difference in the approaches lies in the way these two distributions are calculated. Here, the LexStat method follows Kondrak (2003) in deriving the attested distribution from pairwise alignments. The pairwise alignments are calculated for all possible language pairs in a multilingual word list. In contrast to Kondrak (ibid.), however, LexStat adopts the idea of consistencybased sequence alignment (see Section 3.4.2) in creating a library of pairwise alignments. Instead of computing only global or only local pairwise alignments to derive the attested distribution, both global and local alignments are computed, and the resulting distribution is averaged. The advantage of this approach is that more information can be taken into account, especially when words exhibit rather "local" similarities, i.e., if they, for example, contain prefixes or suffixes.

The problem all approaches have to deal with is how to handle the noise in the data. Regular sound correspondences can only be determined for cognate words, but not all words in the data are cognate. When linguists determine regular sound correspondences manually, they only count the number of correspondences in those words which they assume to be cognate. Other words are discarded from the count. It is useful to model this behaviour by preprocessing the data for probably fruitful matches. In the first version of LexStat (List 2012a), I employed a very simple strategy to account for this: instead of taking all aligned word pairs of each language pair to calculate an attested distribution of correspondence frequencies in pairwise alignments, only those alignments whose SCA distance was beyond a certain threshold were taken into account. The SCA distance (SCAD) was calculated by applying the aforementioned formula of Downey et al. (2008) to the similarity scores produced by the SCA method (see Equation 4.2 on page 142):

$$
S C A D=1-\frac{2 \cdot S_{A B}}{S_{A}+S_{B}}
$$

where $S_{A B}$ is the similarity score of an alignment of two words $A$ and $B$ produced by the SCA method, and $S_{A}$ and $S_{B}$ are the similarity scores produced by the alignment of $A$ and $B$ with themselves. That SCA distances are not a bad heuristic for the preprocessing of word lists, is illustrated in Table 4.22, where the capability of the three variants of SCA distances to discriminate between cognate words is contrasted with the discriminative force of the normalized edit distance (NED), which is calculated by dividing the edit distance 
by the length of the smaller sequence. The discriminative force of the different distance scores was measured by calculating the $n$-point average precision (cf. the description in Kondrak 2002: 118f) on a small dataset of Swadesh-200 word lists containing manually edited cognate judgments from the literature for Albanian, English, French, and German (Kessler 2001). As can be see from the table, all SCA distances largely outperform the normalized edit distance.

In the most recent version of the LexStat method, a new strategy for the preprocessing of word lists is employed. The main idea is to take multilingual instead of bilingual information into account. Given that LexStat is but one of four different ACD methods implemented in LingPy, the preprocessing can also be based on one of the three other methods, i.e. the algorithm can first use a simplified language-independent method to search for cognates in a multilingual word list, and than use these preliminary cognate judgments to calculate an attested distribution of correspondence frequencies. The apparent advantage of this approach is that a multilingual ACD method will usually find more cognates than a bilingual one, since false negatives resulting from high distance scores in one language pair may be levelled by the average distances over multiple languages. It is, for example, difficult - if not impossible - to prove the cognacy of German Zahn [tsa:n] 'tooth' and English tooth [tu: $\theta$ ]

\begin{tabular}{|c|c|c|c|c|c|}
\hline \multirow{2}{*}{ Taxa } & Cogn. & \multirow{2}{*}{ NED } & \multicolumn{3}{|c|}{ SCA distance } \\
\cline { 4 - 6 } & Prop. & & DOLGO & ASJP & SCA \\
\hline \hline German / Albanian & 0.12 & 30.62 & 32.60 & 38.78 & 44.58 \\
\hline German / French & 0.26 & 45.97 & 55.83 & 54.87 & 59.84 \\
\hline German / English & 0.59 & 89.16 & 94.65 & 93.92 & 94.45 \\
\hline Albanian / French & 0.17 & 62.74 & 50.32 & 61.73 & 64.12 \\
\hline Albanian / English & 0.10 & 15.74 & 32.29 & 31.10 & 39.86 \\
\hline French / English & 0.28 & 61.92 & 67.34 & 70.85 & 64.65 \\
\hline \hline Average & 0.25 & 51.03 & 55.50 & 58.54 & 61.25 \\
\hline
\end{tabular}

Table 4.22: $\mathrm{n}$-Point Average Precision of NED and the three variants of SCA distances on Swadesh-200 word lists of four genetically related languages (data taken from Kessler 2001). 
on the basis of Swadesh-200 word lists alone, since there are two gaps in the

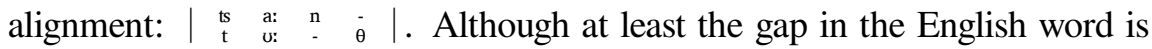
regular (see the examples in Section 4.1.1) and might be detected as such in a Swadesh-200 word list of English and German, the missing dental in the German word has no further counterpart in the list, and aggravates the task of finding a correct scenario for an alignment algorithm. This changes when adding Dutch tand [tant] 'tooth' to the comparison. This word serves as a

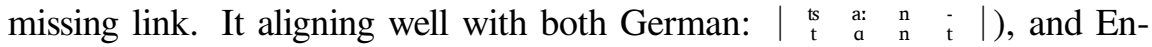

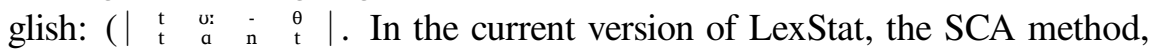
by which sequences are clustered into cognate sets whose average SCA distance is beyond a certain threshold, is used for this purpose, since it performed better than the other two methods compared in List (2012a). For this preliminary stage of cognate detection, it is not important that the algorithm finds only true cognates. What is important, is that the recall is considerably high, i.e. that the algorithm finds many of the cognate pairs in the word lists. Therefore, the threshold for this preprocessing stage is set to 0.6 by default.

In contrast to the creation of the attested distribution of matching residue pairs, the creation of the expected distribution is more complicated. In biology, the expected frequencies of matching residue pairs are usually inferred mathematically, under the assumption that all aligned residue pairs are statistically independent of each other. Unfortunately, this is not possible in the LexStat approach, since the attested frequencies are derived from an alignment algorithm which automatically favors and disfavors certain matches. Thus, no matter whether or not two languages are related, there will always be a large amount of vowel-vowel matches in the attested distribution, vowels will never match with consonants, and certain consonant matchings will always be favored while others are disfavored. In order to derive an unbiased expected distribution, I follow the approach by Kessler (2001: 48-50) in using a Monte Carlo permutation test (see also Baxter and Manaster Ramer 2000). Thus, in order to create the expected distribution of matching residue pairs, the words in the word lists for a given language pair are repeatedly resampled by shuffling one of the entry columns. In each resampling step, pairwise alignments of the new word pairs are carried out, using the same methods that are used for the creation of the attested distribution. The average of the frequencies of the residue pairs in all samples is then taken to reflect the expected frequencies. In the default settings, the number of repetitions is set to 1000 , yet many tests 
showed that even the number of 100 repetitions is sufficient to yield satisfying results that do not vary greatly.

In contrast to most other approaches proposed so far, LexStat does not try to extract the true correspondences in a given dataset, but instead uses the attested and the expected distribution directly to compute log-odds scores which serve as a language-specific scoring matrix for an alignment algorithm. In order to calculate the similarity score $s_{x, y}$ for each residue pair $x$ and $y$ in the dataset, LexStat uses the the formula:

$$
s_{x, y}=\frac{1}{r_{1}+r_{2}}\left(r_{1} \log _{2}\left(\frac{a_{x, y}^{2}}{e_{x, y}^{2}}\right)+r_{2} d_{x, y}\right),
$$

where $a_{x, y}$ is the attested frequency of the segment pair, $e_{x, y}$ is the expected frequency, $r_{1}$ and $r_{2}$ are scaling factors, and $d_{x, y}$ is the similarity score of the original scoring function which was used to retrieve the attested and the expected distributions. This formula combines different approaches from the literature on sequence comparison in historical linguistics and biology. The idea of squaring the frequencies of attested and expected frequencies was adopted from Kessler (2001: 150), reflecting "the general intuition among linguists that the evidence of phoneme recurrence grows faster than linearly". As mentioned above, the calculation of log-odds scores follows the practice in biology to retrieve similarity scores which are apt for the computation of alignment analyses (Henikoff and Henikoff 1992). The incorporation of the alignment score $d_{x, y}$ of the original language-independent scoring-scheme copes with possible problems resulting from small word lists: If the dataset is too small to allow the identification of recurrent sound correspondences, the language-independent alignment scores prevent the method from treating generally probable and generally improbable matchings alike. The ratio of language-specific to language-independent alignment scores is determined by the scaling factors $r_{1}$ and $r_{2}$. In the default settings of LexStat, it is set to 3:2.

As an example of the computation of language-specific scoring schemes, Table 4.23 shows attested and expected frequencies along with the resulting similarity scores for the matching of word-initial and word-final sound classes in the aforementioned test set of Kessler (2001). The word-initial and wordfinal classes $\mathrm{T}=[\mathrm{t}, \mathrm{d}], \mathrm{C}=[\mathrm{ts}], \mathrm{S}=\left[\int, \mathrm{s}, \mathrm{z}\right]$ in German are contrasted with the word-initial and word-final sound classes $T=[t, d]$ and $D=[\theta, \partial]$ in English. As can be seen from the table, the scoring scheme correctly reflects the basic sound correspondences between English and German resulting from 


\begin{tabular}{|c|c||l|c||c|c|c|}
\hline \multicolumn{2}{|c|}{ English } & \multicolumn{2}{c|}{ German } & \multirow{2}{*}{ Att. } & \multirow{2}{*}{ Exp. } & \multirow{2}{*}{ Score } \\
\hline IPA & Cl. & IPA & Cl. & & & \\
\hline \hline \multirow{3}{*}{ t, d } & \multirow{2}{*}{ T } & t, d & T & 3.00 & 1.24 & 5.50 \\
\cline { 3 - 6 } & & ts & C & 3.00 & 0.38 & 5.10 \\
\cline { 3 - 6 } & & S, s, z & S & 1.00 & 1.87 & -2.20 \\
\hline \hline \multirow{3}{*}{$\theta$, ठ } & \multirow{2}{*}{ D } & t, d & T & 7.00 & 0.69 & 5.83 \\
\cline { 3 - 6 } & & ts & C & 0.00 & 0.21 & -2.20 \\
\cline { 3 - 6 } & & s, z & S & 1.00 & 1.64 & -0.60 \\
\hline
\end{tabular}

(a) Word-initial

\begin{tabular}{|c|c||l|c||c|c|c|}
\hline \multicolumn{2}{|c|}{ English } & \multicolumn{2}{c|}{ German } & \multirow{2}{*}{ Att. } & \multirow{2}{*}{ Exp. } & \multirow{2}{*}{ Score } \\
\hline IPA & Cl. & IPA & Cl. & & & \\
\hline \hline \multirow{3}{*}{ t, d } & \multirow{3}{*}{$\mathrm{T}$} & t, d & T & 22.00 & 9.03 & 4.78 \\
\cline { 3 - 6 } & & ts & C & 3.00 & 1.69 & -0.60 \\
\cline { 3 - 6 } & & S, s, z & S & 7.50 & 4.93 & 1.11 \\
\hline \hline \multirow{2}{*}{$\theta, \delta$} & \multirow{2}{*}{ D } & t, d & T & 4.00 & 1.17 & 4.02 \\
\cline { 3 - 6 } & & ts & C & 0.00 & 0.23 & -2.20 \\
\cline { 3 - 6 } & & s, z & S & 0.00 & 0.79 & -0.60 \\
\hline
\end{tabular}

(b) Word-final

Table 4.23: Attested versus expected frequencies of residue pairs and the resulting logodds scores in (a) word-initial and (b) word-final position for German and English in the dataset of Kessler (2001). The attested distribution reflects the average of one global and one local alignment analysis. For the expected distribution, a Monte-Carlo permutation test of 1000 runs was carried out.

the High German Consonant Shift (Trask 2000: 300-302), which is reflected in such cognate pairs as English town [taun] vs. German Zaun [tsaun] 'fence', English thorn [ $\theta$ ว:n] vs. German Dorn [dorn] 'thorn', English dale [deIl] vs. German Tal [ta:l] 'valley', and English hot [hət] vs. German hei $\beta$ [hars] 'hot'. The specific benefit of representing the phonetic segments as tuples consisting of their respective sound class along with their prosodic context also becomes evident: The correspondence of English [t] with German [s] is only attested in word-final position, correctly reflecting the complex change of former $[\mathrm{t}]$ to $[\mathrm{s}]$ 
in non-initial position in German. If it were not for the specific representation of the phonetic segments by both their sound class and their prosodic context, the evidence would be blurred. ${ }^{16}$

\section{Distance Calculation}

Once the language-specific scoring scheme is computed, the distances between all word pairs are calculated. Here, LexStat uses the semi-global alignment algorithm. This modification is useful when words contain prefixes or suffixes which might distort the calculation. The alignment analysis requires no further parameters such as gap penalties, since they have already been calculated in the previous stage. The similarity scores for pairwise alignments are converted to distance scores using the same formula which is used to calculate the language-independent SCA distances (see Equation 4.11 on page 178). This formula does not only require the similarity score between two different sequences as input, but also the similarity score of the sequences compared with themselves. The similarity of one sequence compared with itself is calculated by applying the same Monte Carlo permutation test to all languages compared with themselves and calculating the respective log-odds scores.

The benefits of language-specific in comparison to language-independent sequence distances are illustrated in Figure 4.24. The figure shows the crossproduct of 100 cognates which were randomly chosen from a larger sample of 658 cognate pairs between English and German, extracted from KLUGE. In the figures, four different distance scores are compared: percentage identity (PID, see Equation 3.1 on page 74), converted to a distance score, normalized edit distance (NED), the SCA distance (SCA model), and the LexStat distances, which were computed for the two language pairs. In each plot, the real cognates are given in the diagonal cells from bottom left to top right. All other cells of the matrices reflect unrelated word pairs. A certain "cognate signal" can be detected in all plots. However, the signal is quite weak in the PID and the NED plots. In the SCA plot, the signal is stronger, but blurred by generally low distances between non-cognate word pairs. In the LexStat plot, the signal is the strongest: Most of the cognates receive a distance score around or beyond 0.3 , while most non-cognates receive a considerably higher distance

16 The alignments along with the distance scores for all word pairs between German and English are given in Appendix C.2. 
score. This clearly illustrates the superiority of language-specific distances for the task of distinguishing cognates from non-cognates. ${ }^{17}$

\section{Sequence Clustering}

Distance scores do not directly reflect cognate judgments, since they only indicate the similarity between sequence pairs, but not between whole groups of sequences. Applying a threshold beyond which cognacy is assumed for a
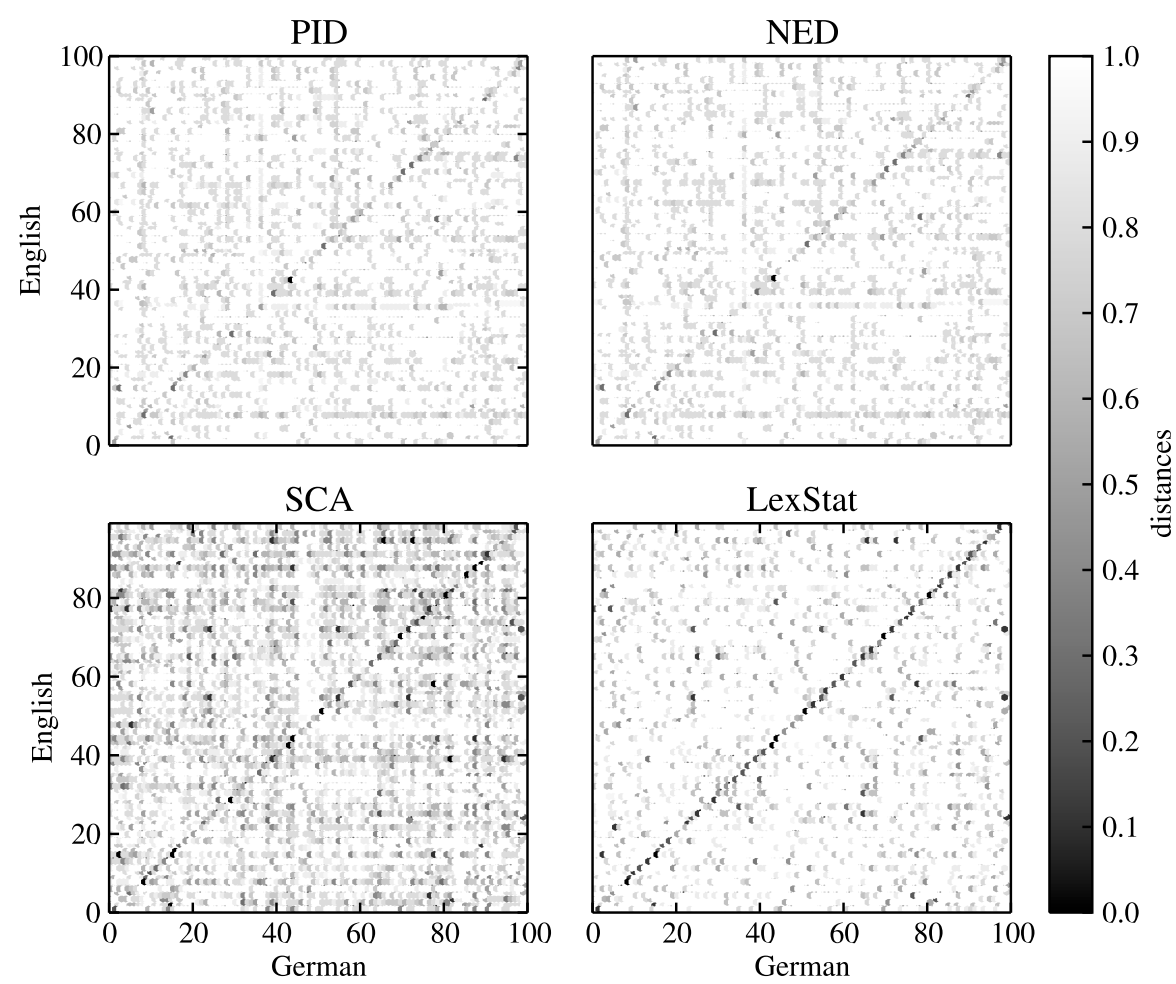

Figure 4.24: Percentage identities (PID), normalized edit distances (NED), SCA distances, and LexStat distances for the cross product of a sample of 100 cognates between English and German.

${ }_{17}$ All distance scores for the 100 cognate pairs are given in Appendix D.1. 
given word pair does not solve the problem, since the resulting cognate decision may create intransitive relations (Hauer and Kondrak 2011: 868f). In order to retrieve transitive cognate judgments, a cluster method has to be applied. LexStat uses a flat cluster variant of the UPGMA algorithm (Sokal and Michener 1958) to cluster all words occurring in the same semantic slot into cognate sets. This flat cluster algorithm was written by the author. In contrast to traditional UPGMA clustering, it terminates when a user-defined threshold of average pairwise distances is reached. The threshold itself reflects the maximally allowed average distance between all word pairs in a given cluster.

Figure 4.25 shows how pairwise distances of German, English, Danish, Swedish, Dutch!Standard Dutch, and Norwegian translations of the basic concept 'woman', taken from the GER dataset (see Table 4.25), are merged into cognate sets by the flat cluster algorithm.

\subsubsection{Evaluation}

\section{Gold Standard}

What was said about benchmark datasets for phonetic alignment in Section 4.2.4 also holds for automatic cognate detection: The number of available gold standards is very limited. Kessler (2001) compiled a dataset in which the 200 basic vocabulary items of Swadesh (1952) are translated into eight languages. Apparently, this dataset has only rarely been used as a gold standard for cognate detection before, and the only approach known to me is only based on the comparison of language pairs (Mackay and Kondrak 2005). The advantage of this dataset is the quality of the material: All words are given in orthography and IPA transcription, the cognate judgments are all substantiated by numerous references taken from the literature, and borrowings are especially marked by the author. The drawback of this dataset is its limited size and the languages chosen by the author. The dataset contains Latin and French, which - taken together - are of little use for the evaluation of ACD methods, since the former is the ancestor of the latter. Because the author also deliberately selected genetically unrelated languages, the number of actual cognate sets in the dataset is considerably low. Thus, although the dataset by Kessler (2001) should surely be considered when testing ACD methods, it is not enough to base the evaluation on this dataset alone.

There are a couple of publicly available lexicostatistical databases which offer manually edited cognate judgments that are supposed to reflect the com- 


\begin{tabular}{|c|c|c|c|c|c|c|}
\hline & $\begin{array}{c}\text { German } \\
\text { Frau }\end{array}$ & $\begin{array}{c}\text { English } \\
\text { woman }\end{array}$ & $\begin{array}{c}\text { Danish } \\
\text { kvinde }\end{array}$ & $\begin{array}{c}\text { Swedish } \\
\text { kvinna }\end{array}$ & $\begin{array}{c}\text { Dutch } \\
\text { vrouw }\end{array}$ & $\begin{array}{c}\text { Norwegian } \\
\text { kvine }\end{array}$ \\
\hline $\begin{array}{c}\text { German } \\
\text { [frau] }\end{array}$ & 0.00 & 0.95 & 0.81 & 0.70 & 0.34 & 1.00 \\
\hline $\begin{array}{c}\text { English } \\
\text { [wumən] }\end{array}$ & 0.95 & 0.00 & 0.78 & 0.90 & 0.80 & 0.80 \\
\hline $\begin{array}{c}\text { Danish } \\
\text { [kvenə] }\end{array}$ & 0.81 & 0.78 & 0.00 & 0.17 & 0.96 & 0.13 \\
\hline $\begin{array}{c}\text { Swedish } \\
\text { [kvin:a }]\end{array}$ & 0.70 & 0.90 & 0.17 & 0.00 & 0.86 & 0.10 \\
\hline $\begin{array}{c}\text { Dutch } \\
\text { [vrau] }\end{array}$ & 0.34 & 0.80 & 0.96 & 0.86 & 0.00 & 0.89 \\
\hline $\begin{array}{c}\text { Norwegia } \\
\text { [kvinə] }\end{array}$ & 1.00 & 0.80 & 0.13 & 0.10 & 0.89 & 0.00 \\
\hline
\end{tabular}

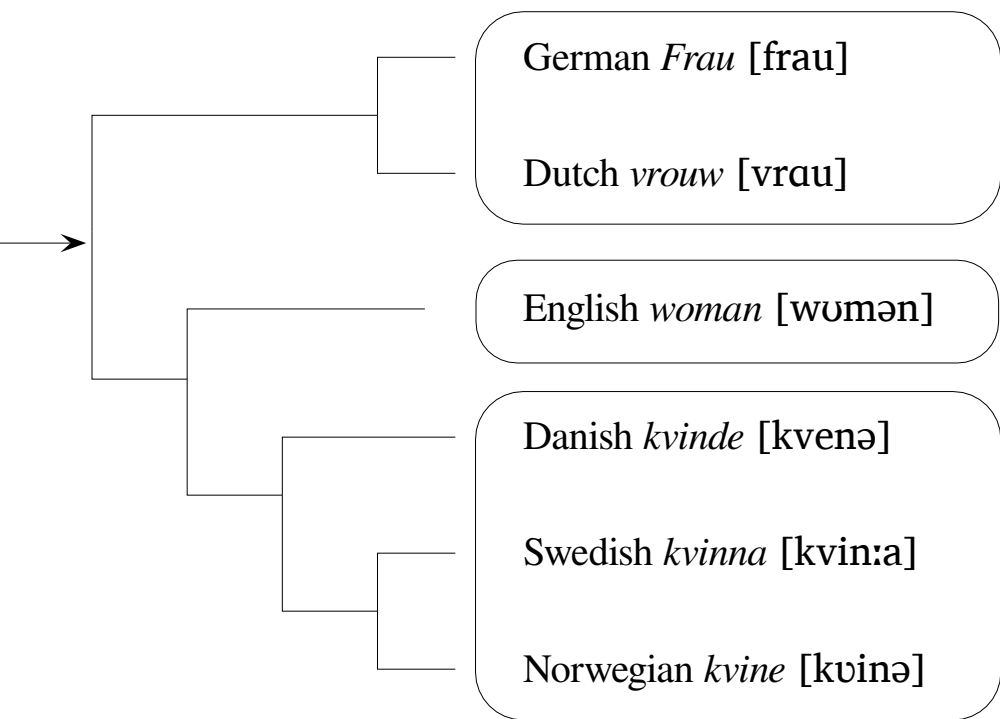

Figure 4.25: The flat clustering variant of the UPGMA algorithm applied to the basic vocabulary item 'woman' in a couple of Germanic languages. The algorithm successively merges words into larger clusters until a user-defined threshold indicating the maximum of the average distances between all word pairs in a cluster is reached. 
munis opinio for a given language family. Among these are the Dyen-KruskalBlack database (DKBD, Dyen et al. 1992) which covers almost 100 IndoEuropean languages, the Indo-European Lexical Cognacy Database (IELex), an improved version of the DKBD, the Tower of Babel database (ToB) and its successor, the Global Lexicostatistical Database (GLD), which cover many different language families, and the Austronesian Basic Vocabulary Database (ABVD, Greenhill et al. 2008). Of all these databases, the DKBD has been most frequently used as a benchmark for various applications (Bergsma and Kondrak 2007, Delmestri and Cristianini 2010, Hall and Klein 2010, Hauer and Kondrak 2011, Mackay and Kondrak 2005). Unfortunately, of all these databases, only parts of the IELex can be directly used as a gold standard for the kind of ACD evaluation that is proposed in this study, since none of the other databases offers true phonetic transcriptions. They are either based on orthography, or employ inconsistent idiosyncratic transcription systems which cannot be directly converted into IPA format. While these databases can be used for methods which do not directly depend on IPA transcriptions as in-

\begin{tabular}{|l|l|l|l|l|l|l|l|}
\hline Dataset & Languages & I & W & CS & $D$ & T & Source \\
\hline \hline BAI & Bai dialects & 110 & 1028 & 205 & 0.10 & 9 & Wang 2006a \\
\hline IEL & $\begin{array}{l}\text { Indo-European } \\
\text { Languages }\end{array}$ & 207 & 4393 & 1778 & 0.38 & 20 & IELex \\
\hline JAP & Japanese dialects & 200 & 1985 & 458 & 0.14 & 10 & Shirō 1973 \\
\hline OUG & Uralic languages & 110 & 2055 & 239 & 0.07 & 21 & GLD \\
\hline PAN & $\begin{array}{l}\text { Austronesian lan- } \\
\text { guages }\end{array}$ & 210 & 4358 & 2730 & 0.61 & 20 & ABVD \\
\hline SIN & Chinese dialects & 140 & 2789 & 1025 & 0.33 & 15 & YINKU \\
\hline
\end{tabular}

Table 4.24: The general partition of the gold standard for the evaluation of methods for automatic cognate detection. Columns I, W, CS, and T show the numbers of items, words, cognate-sets, and taxa. The diversity index $D$ is calculated by dividing the difference between the number of cognate sets and items by the difference between the number of words and items. The lower the value the less diverse are all languages in the respective subset on average. 
put format (Bergsma and Kondrak 2007, Hauer and Kondrak 2011), they are surely not apt for the method proposed in this study.

For the evaluation of an earlier version of the algorithm presented in this study (List 2012a) a specific benchmark dataset was compiled by taking publicly available datasets with authoritative cognate judgments and converting the orthographical entries into IPA. For this study the former benchmark was extended and updated. The new benchmark database for cognate detection can be downloaded from the supporting online material accompanying this study (see Supplementary Material III). In contrast to the benchmark used in List (ibid.), three new subsets were included: The first one (IDS) consists of 550 items translated into four languages (German, English, Dutch, and French) which were taken from the Intercontinental Dictionary Series (IDS). The orthographical entries were converted into IPA transcriptions by the author, and cognate judgments were applied manually. The sources which were considered for transcriptions and cognate judgments are given in Appendix A.1. The second one (PAN) is a small part of the ABVD which was kindly provided by Simon Greenhill. It consists of 20 languages given in a very consistent transcription system that could be easily converted to IPA by the author. The third one (IEL) is a small part of the IELex, kindly provided by Micheal Dunn, consisting of 20 languages for which full phonetic transcriptions were available.

The new benchmark divided into two partitions: one "basic" partition and one "specific" partition. The basic partition consists of the six largest subsets, each reflecting a different language family (see Table 4.24). The specific partition consists of various subsets of different sizes which can be used to test specific characteristics of ACD methods (see Table 4.25). It is useful to get a rough impression regarding the diversity of a given dataset. I propose to calculate it with help of the formula:

$$
D=\frac{C-I}{W-I},
$$

where $C$ is the number of cognate sets, $I$ is the number of basic vocabulary items, and $W$ is the number of language entries (words). This index contrasts the number of cognate sets in a dataset with the number of possible cognate sets. A score of 0 indicates full cognacy, i.e. all words in all semantic slots would be cognate for such a dataset, a high score points to a large amount of unrelated words. As can be seen from the indices for the two partitions of the gold standard, the diversity of the subsets shows a great variation, ranging from dataset with a very high density, such as the OUG data, up to very diverse 


\begin{tabular}{|c|c|c|c|c|c|c|c|}
\hline Dataset & Languages & I & $\mathbf{W}$ & CS & $D$ & $\mathbf{T}$ & Source \\
\hline GER & $\begin{array}{l}\text { Germanic } \\
\text { languages and } \\
\text { dialects }\end{array}$ & 110 & 814 & 182 & 0.10 & 7 & ToB \\
\hline IDS & $\begin{array}{l}\text { Romance and } \\
\text { Germanic } \\
\text { languages }\end{array}$ & 550 & 2429 & 1490 & 0.50 & 4 & IDS \\
\hline KSL & $\begin{array}{l}\text { Various lan- } \\
\text { guages (partially } \\
\text { unrelated) }\end{array}$ & 200 & 1400 & 1179 & 0.82 & 7 & Kessler 2001 \\
\hline PIE & $\begin{array}{l}\text { Indo-European } \\
\text { languages }\end{array}$ & 110 & 2172 & 615 & 0.24 & 19 & ToB \\
\hline ROM & $\begin{array}{l}\text { Romance } \\
\text { language }\end{array}$ & 110 & 589 & 177 & 0.14 & 5 & ToB \\
\hline SLV & Slavic languages & 110 & 454 & 165 & 0.16 & 4 & ToB \\
\hline
\end{tabular}

Table 4.25: The specific partition of the gold standard for the evaluation of methods for automatic cognate detection. Regarding the diversity index $D$, see the description in Table 4.24.

ones, such as the PAN data. This is exactly what is needed for a representative gold standard.

\section{Evaluation Measures}

The ACD task is essentially a clustering task. The evaluation of clustering tasks is much more complicated than the evaluation of simple pairwise classification tasks (Hauer and Kondrak 2011). Most clustering evaluation measures can be assigned to two different perspectives: the set perspective, or the pair perspective. The set perspective treats clusters as indivisible wholes. The pair perspective treats a set of clustered items as a set of pairwise decisions between all items in the set.

Bergsma and Kondrak (2007) test the performance of their ACD method by calculating the set precision (SP), and the set recall (SR). The set precision is defined as the "proportion of sets proposed by [the] system which are also sets in the gold standard" (Bergsma and Kondrak 2007: 659), and can be 
calculated with the formula:

$$
S P=100 \cdot \frac{\left|C_{r} \cap C_{t}\right|}{\left|C_{t}\right|},
$$

where $C_{r}$ is the set of cognate sets in the reference and $C_{t}$ is the number of cognate sets in test set. Accordingly, the set recall is defined as the "proportion of gold standard sets that [the] system correctly proposes" (ibid.), and can be calculated with the formula:

$$
S R=100 \cdot \frac{\left|C_{r} \cap C_{t}\right|}{\left|C_{r}\right|} .
$$

Both set precision and set recall can be combined by calculating their harmonic mean, the set F-score (SF):

$$
S F=2 \cdot \frac{S P \cdot S R}{S P+S R} .
$$

\begin{tabular}{|l||c|c|c|c|}
\hline Words & "BAUCH" & "BELLY" & "BUK" & "MAGE " \\
\hline \hline Reference & 1 & 2 & 1 & 3 \\
\hline Test & 1 & 1 & 1 & 2 \\
\hline
\end{tabular}

(a) Cluster decision

\begin{tabular}{|cc|c|c|}
\hline \multicolumn{2}{|c|}{ Pairs } & Ref. & Test \\
\hline \hline "BAUCH" & "BELLY" & 0 & 1 \\
\hline "BAUCH" & "BUK" & 1 & 1 \\
\hline "BAUCH" & "MAGE" & 0 & 0 \\
\hline "BELLY" & "BUK" & 0 & 1 \\
\hline "BELLY" & "MAGE" & 0 & 0 \\
\hline "BUK" & "MAGE" & 0 & 0 \\
\hline
\end{tabular}

(b) Pairwise perspective

Table 4.26: The pair perspective in clustering evaluation. Clusters (a) are represented by assigning an integer ID to a given cluster. If two words have the same ID, they are assigned to the same cluster. When comparing cluster decisions within the pair perspective (b), all possible pairs of clustered items are considered, and cluster decisions are displayed by 1 (in the same cluster) and 0 (in different clusters). 
The set scores are a very conservative evaluation measure, since only identical clusters in reference and test set count positively while identical tendencies are ignored. As a result, the performance of an algorithm may be blurred, especially when the test data is considerably diverse. As an alternative one can turn to the pair perspective which, instead of checking for identical clusters, checks for the identity of pairwise decisions implied by a cluster, as illustrated in Table 4.26. In List (2012a) I used the pair perspective to calculate the proportion of identical decisions (ID score). Following the contingency table in Table 4.27, this score can be calculated by dividing the number of true positives and true negatives by the total number of decisions:

$$
I D=\frac{a+d}{a+b+c+d} .
$$

However, this measure suffers from the shortcoming that it is extremely dependent on the size of the clusters in the dataset. For example, if a dataset contains only a small amount of cognate sets, a poorly performing algorithm which fails to identify most of the cognates may still receive a considerably high ID score as long as it only correctly identifies the non-cognates. In order to avoid these problems, it seems more useful to calculate the pair precision (PP) and the pair recall, which are formally identical with pair precision and pair recall as evaluation measures for alignment analyses introduced in Section 4.2.4. Thus, given all pairwise decisions in reference and test set, the pair precision can be defined as:

$$
P P=100 \cdot \frac{\left|P_{t} \cap P_{r}\right|}{\left|P_{t}\right|},
$$

\begin{tabular}{|c|c|c|}
\hline & $\begin{array}{c}\text { Cognate } \\
\text { Reference }\end{array}$ & $\begin{array}{c}\text { Non-Cognate } \\
\text { Reference }\end{array}$ \\
\hline $\begin{array}{c}\text { Cognate } \\
\text { Test }\end{array}$ & true positives & false positives \\
\hline $\begin{array}{c}\text { Non-Cognate } \\
\text { Test }\end{array}$ & false negatives & true negatives \\
\hline
\end{tabular}

Table 4.27: Pairwise decisions in reference and test set 
where $P_{t}$ is the set of all cognate pairs in the test set and $P_{r}$ is the set of all cognate pairs in the reference set. Note that this is equivalent to calculating:

$$
P P=100 \cdot \frac{a}{a+b},
$$

when applying the contingency table in Table 4.27. The pair precision gives an account on the number of false positives produced by an algorithm: The higher the pair precision, the lower the number of false positive decisions made by an algorithm. Accordingly, the pair recall can be calculated with the formula:

$$
P R=100 \cdot \frac{\left|P_{t} \cap P_{r}\right|}{\left|P_{r}\right|},
$$

which is again identical to:

$$
P R=100 \cdot \frac{a}{a+c} .
$$

The pair recall gives an account on the number of false negatives produced by an algorithm: The higher the pair recall, the lower the number of false negative cognate decisions made by an algorithm. Accordingly, the pair F-score (PFS) can be calculated by the formula:

$$
P F S=2 \cdot \frac{P P \cdot P R}{P P+P R} .
$$

Although the pair scores, as they were defined above, show a clear improvement over both the set scores and the ID score, they are still intimately dependent on the cluster size (Amigó et al. 2009). In order to avoid this problem, Hauer and Kondrak (2011) test their ACD method by calculating B-Cubed scores. B-Cubed scores were originally introduced as part of an algorithm by Bagga and Baldwin (1998), but Amigó et al. (2009) could show that they are especially apt as a clustering evaluation measure. In B-Cubed metrics, precision and recall are computed for each word in the dataset taken as a separate item. The $B$-Cubed precision (BP) for one word $w$ in the dataset can be defined as:

$$
B P_{w}=100 \cdot \frac{\left|R_{w} \cap T_{w}\right|}{\left|T_{w}\right|}
$$


where $R_{w}$ is the cognate set to which $w$ is assigned by the reference set, and $T_{w}$ is the cognate set to which $w$ is assigned in the test set. Accordingly, the $B$-Cubed recall (BR) for one word $w$ in the dataset can be defined as:

$$
B R_{w}=100 \cdot \frac{\left|R_{w} \cap T_{w}\right|}{\left|R_{w}\right|},
$$

and the B-Cubed F-score (BF) can be calculated with help of the formula:

$$
B F_{w}=2 \cdot \frac{B F_{w} \cdot B R_{w}}{B F_{w}+B R_{w}} .
$$

The B-Cubed scores for the whole dataset are calculated by taking the average of the separate scores for each word.

The LingPy library, which I wrote for this study, offers a module which calculates all above-mentioned scores for a given reference and test set (see the documentation for details). From the literature it is not clear, how singletons (words that are not cognate with any other word) should be treated. While in the pair scores they are excluded per definitionem, it seems that they are usually calculated for the set and the B-Cubed scores. However, while it seems understandable to include singletons in the set calculations, since a correctly assigned singleton should certainly score somehow in the evaluation, this is less true for the B-Cubed scores, because they always yield 1, and no further discrimination between true singletons and false singletons is made. Nevertheless, since the literature remains silent regarding this point, singletons are generally included in the calculations produced by LingPy.

\section{Results}

All results which will be reported in the following were achieved by using the most recent version of LexStat along with the same parameters for the different parts of the analysis. For the creation of the language-specific scoring function, attested and expected distributions were calculated by carrying out global and local alignment analyses of the sequences in all language pairs, using the SCA model, and the default SCA scoring function. The GOP was set to -2 for global and to -1 for local alignment analyses. For the creation of the attested distribution, the threshold for the preprocessing was set to 0.6 . The ratio between language-specific and language-independent similarity scores was set to $3: 2$. 
Along with the language-specific LexStat method, three language-independent methods, which are all implemented as part of the LingPy library, were tested: The method by Turchin et al. (2010) (Turchin), a simple ACD method based on the normalized edit distance (NED), and the aforementioned ACD method based on SCA distances (SCA), which was also used for the preprocessing. Since the cognate judgments produced by the Turchin method are either positive or negative, no further clustering of words into cognate sets is required. For the NED and SCA methods, the cognate judgments are produced by applying the same clustering method which is also used for the LexStat procedure to the distance scores produced by the methods. Using a "good" threshold is crucial for the performance of all methods. In order to guarantee comparability regarding specific strengths and weaknesses of the different methods, I decided to "anchor" all thresholds to the average B-Cubed precision received by the Turchin method for the general partition of the gold standard, since it doesn't require a threshold. As a result, the thresholds for NED, SCA, and LexStat were set to $0.65,0.2$, and 0.55 , respectively. All results of the analyses which are discussed in the following are available as part of the supporting online material accompanying this study (see Supplementary Material IV).

General Results Table 4.28 and Figure 4.26 summarize the results of the ACD methods for the basic partition of the gold standard. As can be seen from the table and the figure, the language-specific LexStat method largely outperforms all language-independent methods, in all "unanchored" evaluation measures apart from the Pair precision, where LexStat performs slightly worse than the SCA method. Furthermore, the language-independent SCA method outperforms the two remaining methods. The Turchin method shows the worst performance. Nevertheless, the NED method is only slightly better. The intention of the authors of this method was to derive a method which is considerably conservative and avoids false positives, and this is certainly guaranteed by the method, although it, unfortunately, misses many cognates.

Being conservative rather then liberal when it comes to cognate judgments is an important requirement for ACD methods. Historical linguists usually have the feeling that false positives are worse than false negatives. Given that the for the three threshold-based methods, the precision was anchored to a value which is allows only a small amount of false positives, the remaining question is, how well the methods identify true cognates. Judging from the results on the 


\begin{tabular}{|c|c||c|c|c|c|}
\hline \multirow{2}{*}{ Measure } & \multicolumn{1}{|c||}{ Score } & \multicolumn{4}{c|}{ Method } \\
\cline { 3 - 6 } & & Turchin & NED & SCA & LexStat \\
\hline \hline \multirow{3}{*}{ Pair-Scores } & ID-Score & 75.26 & 77.31 & 82.13 & 84.46 \\
\cline { 2 - 6 } & Precision & 81.39 & 84.09 & 85.96 & 85.91 \\
\cline { 2 - 6 } & Recall & 47.71 & 51.51 & 60.85 & 66.61 \\
\cline { 2 - 6 } & F-Score & 59.48 & 62.32 & 70.50 & 72.19 \\
\hline \hline \multirow{3}{*}{ B-Cubed Scores } & Precision & 89.83 & 90.01 & 90.36 & 90.30 \\
\cline { 2 - 6 } & Recall & 67.23 & 68.63 & 75.92 & 80.58 \\
\cline { 2 - 6 } & F-Score & 76.81 & 77.11 & 82.45 & 84.83 \\
\hline \hline \multirow{3}{*}{ Set-Scores } & Precision & 33.35 & 34.08 & 42.78 & 49.64 \\
\cline { 2 - 6 } & Recall & 46.83 & 46.75 & 52.99 & 57.73 \\
\cline { 2 - 6 } & F-Score & 38.60 & 38.72 & 47.16 & 53.07 \\
\hline
\end{tabular}

Table 4.28: Average scores for the subsets of the gold standard

general partition of the gold standard, it seems to make a difference, whether one compares simple phonetic sequences, as done by the NED method, or complex ones, as done by SCA. Given that Turchin is also based on sound classes, it further seems to make a differences whether one uses alignment analyses, or a simplified matching scheme. Last not least, the language-specific component of LexStat helps to identify a lot more cognates than could be done by the language-independent methods.

As a result of the adjustments of the thresholds to the high B-Cubed precision predefined by the Turchin method, all methods show a higher precision than recall in the pair and the B-Cubed measures. For the set scores the precision is lower than the recall. This is, however, not surprising, since in contrast to the pair and B-Cubed measures, a low set precision doesn't directly point to a high amount of false positives. It may instead also result from a large amount of singletons proposed by an algorithm, since - within the set perspective a singleton also counts as a set. Therefore, a low set precision - i.e. a low proportion of cognate sets proposed by an algorithm which are also reflected in the gold standard - may also refer to a rather large amount of undetected cognate relations, and - judging from the pair and B-Cubed measures - this seems to be exactly the case. 


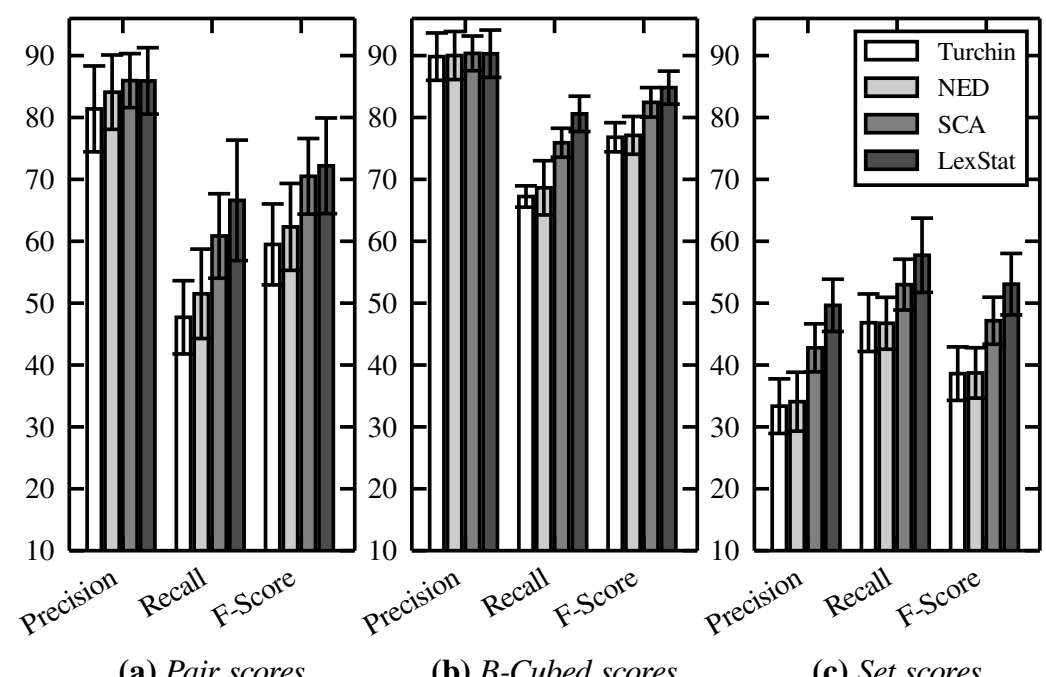

Figure 4.26: Average scores for the subsets of the gold standard

Specific Results The general results suggest the superiority of the languagespecific LexStat approach compared to the language-independent approaches. An important question is, however, whether these results are also consistent throughout all individual subsets of the gold standard, or whether the superiority applies only to a small part of them. The F-scores for the specific results on the subsets of the general partition of the gold standard are given in Table 4.29. As one can see, the LexStat method again performs best in almost all subsets, the only exceptions being the pair F-scores for the IEL subset and the set F-score for the SIN subset, where the SCA method outperforms LexStat, and the pair F-scores for the PAN subset, where LexStat is outperformed by NED and SCA.

The B-Cubed F-scores which are already given in Table 4.29 are also plotted in Figure 4.27, clearly illustrating that LexStat performs best throughout all subsets. What can also be seen from the figure are large differences in the scores received by the methods in dependence of the subset. This becomes less surprising, when taking into account that the subsets differ greatly regarding the diversity of the languages and the time depth separating them. Thus, 


\begin{tabular}{|c|c||c|c|c|c|}
\hline \multirow{2}{*}{ Subset } & \multicolumn{2}{|c|}{ Score } & \multicolumn{4}{c|}{ Method } \\
\cline { 3 - 6 } & & Turchin & NED & SCA & LexStat \\
\hline \hline \multirow{3}{*}{ BAI } & Pair-F-Score & 73.10 & 68.57 & 84.05 & 86.91 \\
\cline { 2 - 6 } & B-Cubed F-Score & 80.04 & 75.43 & 87.16 & 88.72 \\
\cline { 2 - 6 } & Set-F-Score & 31.10 & 24.48 & 44.18 & 45.02 \\
\hline \hline \multirow{3}{*}{ IEL } & Pair-F-Score & 46.53 & 36.52 & 53.23 & 49.40 \\
\cline { 2 - 6 } & B-Cubed F-Score & 75.31 & 72.81 & 79.10 & 80.62 \\
\cline { 2 - 6 } & Set-F-Score & 42.74 & 45.12 & 51.64 & 58.60 \\
\hline \hline \multirow{3}{*}{ OUG } & Pair-F-Score & 74.34 & 83.09 & 83.48 & 91.48 \\
\cline { 2 - 6 } & B-Cubed F-Score & 81.26 & 86.78 & 87.20 & 92.50 \\
\cline { 2 - 6 } & Set-F-Score & 38.51 & 43.33 & 44.84 & 58.55 \\
\cline { 2 - 6 } & Pair-F-Score & 77.31 & 82.06 & 86.85 & 90.01 \\
\cline { 2 - 6 } & B-Cubed F-Score & 81.80 & 85.58 & 88.80 & 91.59 \\
\hline \hline \multirow{3}{*}{ PAN } & Set-F-Score & 38.46 & 45.11 & 53.02 & 63.34 \\
\cline { 2 - 6 } & Pair-F-Score & 35.96 & 55.64 & 50.68 & 45.38 \\
\cline { 2 - 6 } & B-Cubed F-Score & 77.52 & 77.12 & 80.09 & 80.74 \\
\cline { 2 - 6 } & Set-F-Score & 57.63 & 49.09 & 59.45 & 63.31 \\
\hline \hline & Pair-F-Score & 49.67 & 48.04 & 64.68 & 69.98 \\
\cline { 2 - 6 } SIN & B-Cubed F-Score & 64.90 & 64.94 & 72.32 & 74.78 \\
\cline { 2 - 6 } & Set-F-Score & 23.19 & 25.21 & 29.83 & 29.57 \\
\hline
\end{tabular}

Table 4.29: Specific results ( $F$-scores) for the six subsets of the general partition of the gold standard.

the BAI, the JAP, and the OUG subset all consist of languages and dialects which separated not too long ago, while IEL an PAN represent large language families with a considerably long history of divergence. This difference is also reflected in the diversity index of the languages given in Table 4.24 above: The indices of BAI (0.10), JAP (0.14), and OUG (0.07) are all very low compared to those for IEL (0.38), and PAN (0.61). One would expect, however, that the algorithms perform better on the SIN dataset, since the Sinitic languages did not diverge too long ago. Nevertheless, the Sinitic data is highly diverse, as reflected by the high diversity index (0.33), and the fact that the glosses in the word lists of the SIN dataset do not entirely belong to the realm of basic vocabulary but also reflect "cultural" words which are much more prone to change. Furthermore, the data is especially challenging, since in the Chinese 


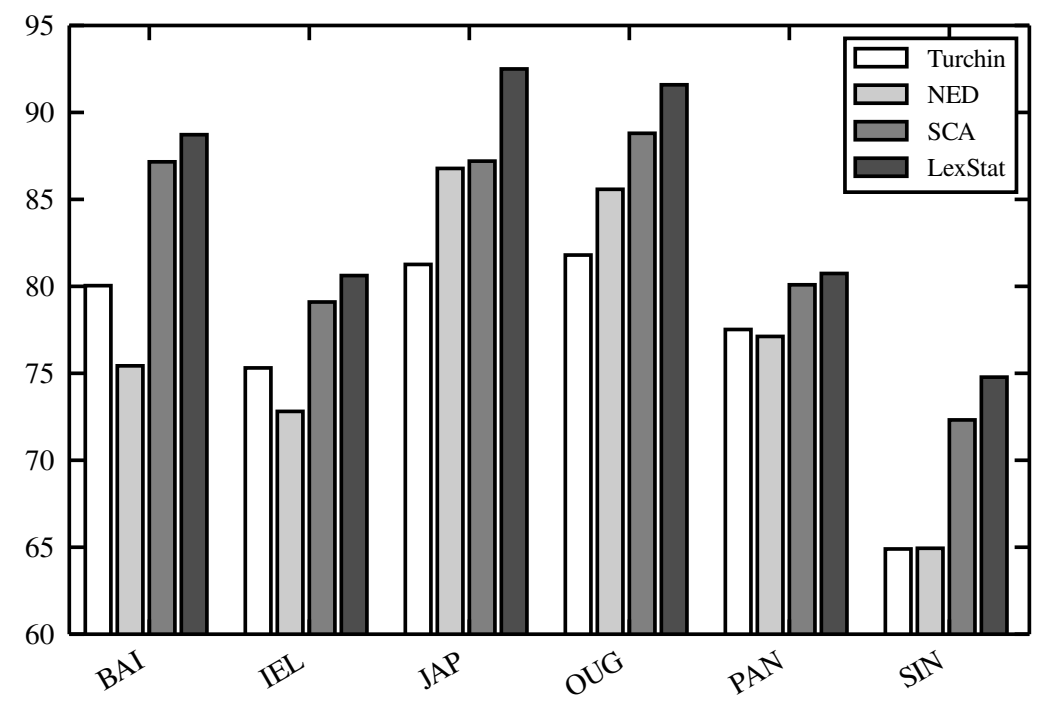

Figure 4.27: $B$-Cubed F-scores for the subsets of the general partition of the gold standard.

dialects there are many compound words which are only partially cognate, i.e. they have morphemes that are cognate, but the compound as a whole is not. Such cases were coded as non-cognate in the data and can easily betray the algorithms.

Time Depth and Diversity The results on the subsets of the general partition suggest that the accuracy of all methods somehow depends on the diversity of the languages being investigated. Following up this question, a further analysis was carried out. This time, the PIE dataset from the specific partition of the gold standard, and its three subsets, GER, ROM, and SLV, were analyzed, using the four methods and the same settings (including the thresholds) as in the previous analyses. The three subsets are directly extracted from the PIE dataset. Thus, their gold standard cognate assignments differ only regarding the number of languages from the PIE data. However, since the subsets reflect the rather "young" Germanic, Romance, and Slavic language families, the time depth that separates the languages in the subsets is much more shallow than 
that of the whole Indo-European language family reflected in the PIE dataset. This is also confirmed by the low diversity indices of the subsets $(0.10,0.14$, and 0.16 for GER, ROM and SLV) compared to the index of the combined and extended dataset $(0.24$, see Equation 4.13 on page 188$)$. The goal of this analysis was to test to which degree time depth and diversity may influence the accuracy of the methods.

The results for the analyses (B-Cubed scores) are given in Table 4.30. As can be seen from the F-scores in the table, diversity and time depth have a crucial impact on the results of the analyses. This is surely no great surprise, since it is well-known that it is easier to compare genetically closely related languages than to compare distantly related ones. However, what might be interesting is that there is only a slight increase in precision, while recall increases greatly. Thus, the majority of problems one faces when entering more shallow time depths in language comparison is not provoked by false positives, but by undetected remote cognate relations.

One further point which is interesting in this context is that in shallow time depths the greatest increase in recall can be recorded for both LexStat (plus 19.9 points on average), followed by NED (15.2), SCA (14.9) and Turchin

\begin{tabular}{|c|c||c|c|c|c|}
\hline \multirow{2}{*}{ Partition } & \multicolumn{2}{|c||}{ Score } & \multicolumn{4}{c|}{ Method } \\
\cline { 3 - 6 } & & Turchin & NED & SCA & LexStat \\
\hline \hline \multirow{3}{*}{ PIE } & B-Cubed Precision & 72.59 & 80.67 & 79.00 & 93.48 \\
\cline { 2 - 6 } & B-Cubed Recall & 58.69 & 54.34 & 66.68 & 74.21 \\
\cline { 2 - 6 } & B-Cubed F-Score & 64.90 & 64.94 & 72.32 & 82.74 \\
\hline \hline \multirow{3}{*}{ GER } & B-Cubed Precision & 97.02 & 98.18 & 97.24 & 94.09 \\
\cline { 2 - 6 } & B-Cubed Recall & 68.11 & 61.24 & 78.97 & 93.42 \\
\cline { 2 - 6 } & B-Cubed F-Score & 80.04 & 75.43 & 87.16 & 93.75 \\
\hline \hline \multirow{3}{*}{ ROM } & B-Cubed Precision & 88.14 & 95.02 & 91.14 & 96.09 \\
\cline { 2 - 6 } & B-Cubed Recall & 65.75 & 59.02 & 69.87 & 91.66 \\
\cline { 2 - 6 } & B-Cubed F-Score & 80.04 & 75.43 & 87.16 & 93.75 \\
\hline \hline \multirow{3}{*}{ SLV } & B-Cubed Precision & 98.28 & 95.34 & 95.56 & 98.30 \\
\cline { 2 - 6 } & B-Cubed Recall & 69.26 & 79.63 & 80.19 & 97.28 \\
\cline { 2 - 6 } & B-Cubed F-Score & 80.04 & 75.43 & 87.16 & 93.75 \\
\hline
\end{tabular}

Table 4.30: Comparing the B-Cubed scores achieved by the methods on the PIE dataset with those achieved on the three less diverse subsets of PIE (GER, ROM, and SLV). 


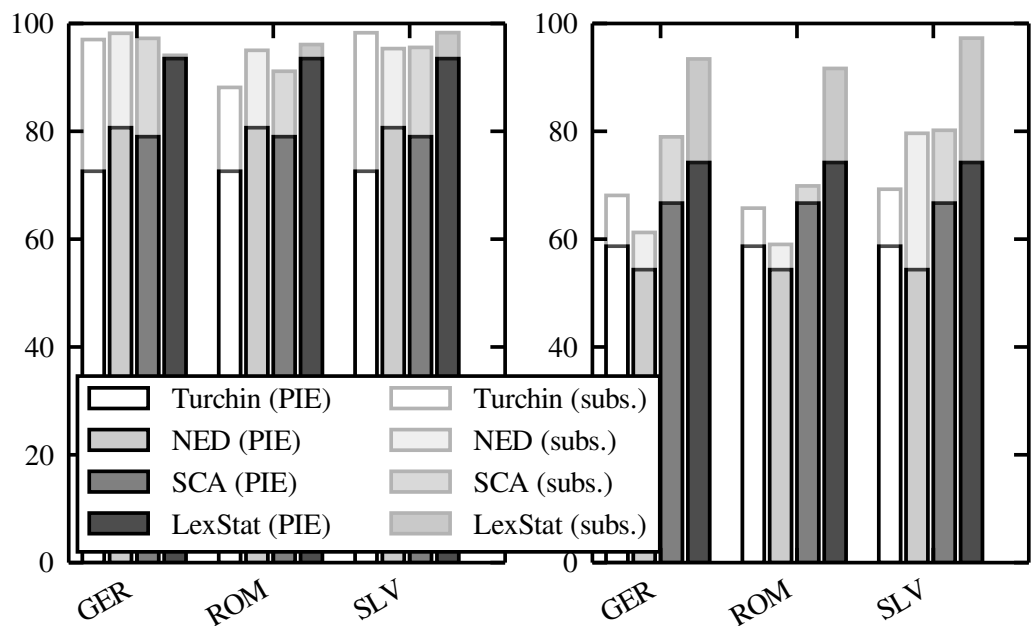

(a) B-Cubed precision.

(b) $B$-Cubed recall.

Figure 4.28: Comparing the B-Cubed precision and recall achieved by the methods on the PIE dataset with the B-Cubed precision and recall achieved on the three less diverse subsets of PIE (GER, ROM, and SLV).

(9.2). The reason for this increase seems to lie in the fact that genetically closely related languages share a higher amount of cognates than genetically distantly related languages. Thus, there is more signal available which is relevant for methods that rely on language-specific, correspondence-based similarities, such as LexStat. Since genetically closely related languages are also phenotypically more similar, there is also more signal available which is relevant for methods that rely on surface similarities, such as NED.

Sample Size It has been stated above that the sample size is of crucial importance for the comparative method. One can generalize this statement and claim that sample size is of crucial importance for all language-specific methods. In order to test this claim, an analysis of different, randomly created partitions of the IDS dataset was carried out. With its 550 glosses translated into four languages, the IDS is the largest dataset in the gold standard with respect to sample size. The data for this test was created as follows: Starting 
from the basic IDS dataset containing all 550 items, 110 new subsets of the data were created by reducing the data stepwise. In each iteration step, 5 items were randomly deleted from the previous dataset. This process was repeated five times, yielding 550 datasets, covering the whole range of possible sample sizes between 5 and 550 in steps of 5 . These datasets were then analysed, using the same settings for the algorithms, as in the analyses reported before.
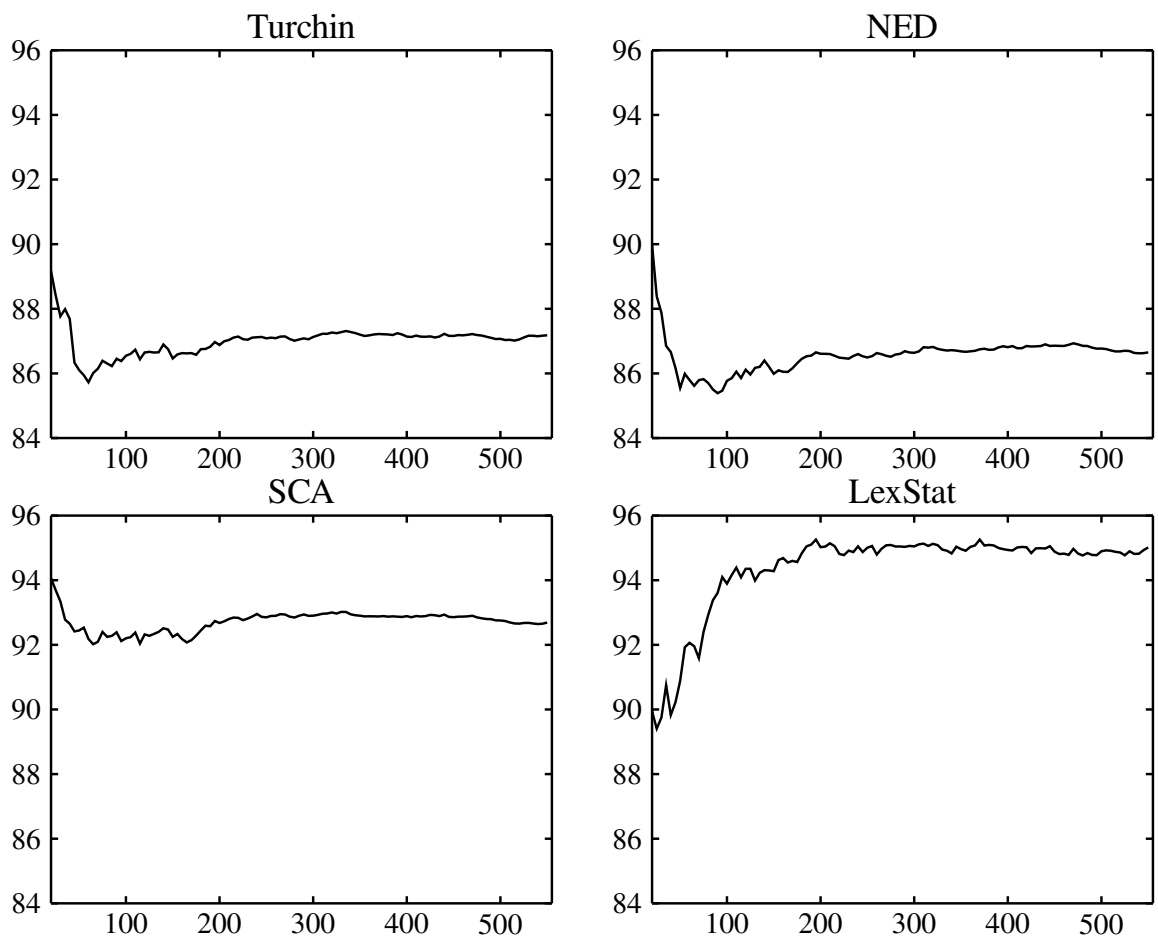

Figure 4.29: Performance of the ACD methods in dependence of the sample size (number of "basic" vocabulary items per word list). The figures show the B-Cubed recall for the four methods. 
For each specific sample size, the average B-Cubed recall for all five subsets was calculated. ${ }^{18}$

The results of this analysis are plotted in Figure 4.29. As can be seen from the figure, the results of the three language-independent methods are quite similar regarding their tendency. Only the degrees of the scores differ. The scores themselves show only marginal variations and remain constant regardless of the sample size. The results for the language-specific LexStat analysis, on the other hand, clearly depend on the sample size, growing logistically, until converging around a sample size of 200 items. This nicely reflects the language-specific character of the LexStat method: If the word-lists fed to the algorithm are too small, no language-specific similarities can be inferred, and no cognates can be detected, as reflected by the low recall for small word lists. This changes dramatically once the sample size is increased. Comparing the scores for a sample size of 50 items (90.88) with those of 100 items (93.89), an increase of about 3 points can be attested, and between 100 and 200 items (95.02), there is still an increase of more than 1 point (see Table 4.31).

One might wonder whether the fact that the scores converge at a sample size of 200 allows to conclude that 200 words are enough for the preliminary stages

\begin{tabular}{|l||c|c|c|c|}
\hline \multirow{2}{*}{ Items } & \multicolumn{4}{|c|}{ B-Cubed Recall } \\
\cline { 2 - 5 } & Turchin & NED & SCA & LexStat \\
\hline \hline 50 & 86.10 & 85.55 & 92.44 & 90.88 \\
\hline 100 & 86.55 & 85.77 & 92.20 & 93.89 \\
\hline 200 & 86.88 & 86.61 & 92.68 & 95.02 \\
\hline 300 & 87.13 & 86.64 & 92.90 & 95.05 \\
\hline 400 & 87.14 & 86.81 & 92.89 & 94.94 \\
\hline 500 & 87.07 & 86.77 & 92.75 & 94.90 \\
\hline
\end{tabular}

Table 4.31: $B$-Cubed recall $(B R)$ of the four different $A C D$ methods in randomly created subsamples of varying sample size extracted from the IDS dataset.

${ }_{18}$ In order to guarantee comparability, the thresholds were again "tuned" on the results of the Turchin method, using the whole IDS dataset for the fine-grading, in order to achieve a similar precision between all models. This lead to thresholds of 0.58 for LexStat, 0.18 for SCA, and 0.65 for NED. 
of language comparison. Since, to my knowledge, the IDS dataset is the only available gold standard covering more than 500 items, it may be questioned whether the data is representative enough to draw general conclusions regarding the necessary size of word lists for ACD methods. Nevertheless, what the results of the analysis show is that word list size indeed has an impact on the results. Thus, when using language-specific methods, there is no use in taking word lists with less than 100 items, and - as the next paragraph will show when seeking for very remote genetic relationships, 200 words are surely not enough.

Proof of Language Relationship So far, I have presented and discussed the results of the new method for automatic cognate detection. Given that the method works quite well, apparently avoiding false positives while at the same time finding more true cognates than alternative methods, one question that surely comes to mind is whether this method is also capable to prove that two languages are genetically related. In order to test the capability of the method to distinguish related from unrelated languages, the KSL dataset (Kessler 2001) is especially apt, since the compiler of the dataset deliberately chose to include genetically unrelated languages. Thus, apart from the four related Indo-European languages Albanian, English, French, and German, the author also included the three mutually unrelated languages Hawaiian, Navajo, and Turkish.

In Table 4.32 the shared cognates between all language pairs as postulated by the four methods (lower triangle) are contrasted with the cognates postulated by the gold standard (upper triangle). ${ }^{19}$ Note that borrowings, which are indicated by Kessler, have been included in this comparison, thus "cognates" in this context are understood as etymologically related words. From a first glance, one can see that all four methods "fail" in so far as they all postulate far more cognates or homologues between unrelated languages than there are in the gold standard. However, the methods fail differently. The Turchin method, which - as could be seen in the previous analyzes - is a very conservative method with a high precision, finds a maximum of 9 cognates in the set of unrelated languages (between Albanian and Hawaiian), while all related languages receive considerably higher scores. One may therefore argue that these false positives do not necessarily invalidate the method, since they can easily be crossed out once genetic relationship is only assumed for languages

${ }^{19}$ The results for this test are given in detail in Appendix D.2. 


\begin{tabular}{|c||c|c|c|c|c|c|c|}
\hline & Albanian & English & French & German & Hawaiian & Navajo & Turkish \\
\hline \hline Albanian & & 20 & 33 & 25 & 0 & 0 & 1 \\
\hline English & 16 & & 56 & 118 & 1 & 0 & 0 \\
\hline French & 23 & 16 & & 51 & 0 & 0 & 0 \\
\hline German & 18 & 82 & 19 & & 0 & 0 & 0 \\
\hline Hawaian & 9 & 3 & 4 & 2 & & 0 & 0 \\
\hline Navajo & 4 & 4 & 3 & 1 & 0 & & 0 \\
\hline Turkish & 7 & 3 & 3 & 1 & 2 & 5 & \\
\hline
\end{tabular}

(a) Turchin

\begin{tabular}{|c||c|c|c|c|c|c|c|}
\hline & Albanian & English & French & German & Hawaiian & Navajo & Turkish \\
\hline \hline Albanian & & 20 & 33 & 25 & 0 & 0 & 1 \\
\hline English & 5 & & 56 & 118 & 1 & 0 & 0 \\
\hline French & 19 & 16 & & 51 & 0 & 0 & 0 \\
\hline German & 9 & 64 & 12 & & 0 & 0 & 0 \\
\hline Hawaian & 2 & 2 & 5 & 3 & & 0 & 0 \\
\hline Navajo & 1 & 1 & 2 & 2 & 1 & & 0 \\
\hline Turkish & 10 & 5 & 5 & 2 & 1 & 3 & \\
\hline
\end{tabular}

(b) $N E D$

\begin{tabular}{|c||c|c|c|c|c|c|c|}
\hline & Albanian & English & French & German & Hawaiian & Navajo & Turkish \\
\hline \hline Albanian & & 20 & 33 & 25 & 0 & 0 & 1 \\
\hline English & 9 & & 56 & 118 & 1 & 0 & 0 \\
\hline French & 19 & 26 & & 51 & 0 & 0 & 0 \\
\hline German & 10 & 103 & 18 & & 0 & 0 & 0 \\
\hline Hawaian & 5 & 3 & 10 & 3 & & 0 & 0 \\
\hline Navajo & 5 & 8 & 4 & 4 & 4 & & 0 \\
\hline Turkish & 7 & 8 & 6 & 4 & 3 & 7 & \\
\hline
\end{tabular}

(c) $S C A$

\begin{tabular}{|c||c|c|c|c|c|c|c|}
\hline & Albanian & English & French & German & Hawaiian & Navajo & Turkish \\
\hline \hline Albanian & & 20 & 33 & 25 & 0 & 0 & 1 \\
\hline English & 3 & & 56 & 118 & 1 & 0 & 0 \\
\hline French & 11 & 18 & & 51 & 0 & 0 & 0 \\
\hline German & 6 & 99 & 11 & & 0 & 0 & 0 \\
\hline Hawaian & 1 & 0 & 0 & 0 & & 0 & 0 \\
\hline Navajo & 0 & 1 & 2 & 1 & 0 & & 0 \\
\hline Turkish & 2 & 0 & 1 & 0 & 0 & 1 & \\
\hline
\end{tabular}

(d) LexStat

Table 4.32: Results of the four methods on the KSL test set. The upper triangle gives the number of shared homologues (cognates and borrowings) in the gold standard, the lower triangle gives the number of shared homologues as determined by the methods. 
sharing more than a certain amount of "sound-class-cognates". The NED method, on the other hand, finds more cognates between Albanian and Turkish, than between Albanian and English. Given the generally rather high number of matches between Turkish and the Indo-European languages produced by NED, the method suggests that Turkish is an Indo-European language. A similar scenario is drawn by the SCA method which postulates quite a few cognates for the unrelated languages. LexStat postulates the lowest number of cognates for the unrelated languages. However, the high precision of the method comes with a cost, since the status of Albanian as an Indo-European language is now no longer evident. However, that it is difficult - if not impossible - to prove that Albanian is an Indo-European language when relying on small word lists of 200 items only, is not a specific problem of automatic methods, but applies also to the comparative method.

\subsubsection{Examples}

For reasons of convenience, all examples which are given in the following are drawn from the analysis of the KSL dataset (Kessler 2001). Since the data for the results of all methods on this test set is also given in Appendix D.2, I won't dive too deep into the details here, but only point to some general tendencies which can be easily spotted when comparing the concrete cluster decisions of the algorithms. All examples given in the following follow the same structure. Cognate decisions are displayed with help of integers assigned to each language entry for an item of a multilingual Swadesh list. If the integers are the same for two or more entries, the respective method assigns the entries to the same cluster and therefore judges the words to be etymologically related or cognate. Cluster decisions where the methods differ from the gold standard decisions are especially marked by shading the cells which differ in gray.

Note that the KSL dataset gives the phonetic sequences in different formats. The user can choose between three levels of representation: word, stem, and root. Since the representation on the word level in the KSL data is not optimal for automatic comparison (employing the third person singular for verbs), and the root representation reflects deep historical knowledge of the languages, the stem representation was used for this analysis. Since the data was not further modified, I will use the same orthographic and phonetic representations which can be found in the original data. Since the different representation levels are only applied to the phonetic transcriptions, the orthographic entries in the data represent verbs by using their third person singular form. 


\begin{tabular}{|l|l|l||c||c|c|c|c|}
\hline \multicolumn{3}{|c|}{ "dig” (30) } & GLD & TUR & NED & SCA & LxS \\
\hline \hline Albanian & gërmon & gərmo & 1 & 1 & 1 & 1 & 1 \\
\hline English & digs & dıg & 2 & 2 & 2 & 2 & 2 \\
\hline French & creuse & krøze & 3 & 1 & 3 & 3 & 3 \\
\hline German & gräbt & gra:b & 4 & 1 & 1 & 3 & 4 \\
\hline Hawaiian & 'eli & Peli & 5 & 5 & 5 & 5 & 5 \\
\hline Navajo & hahashgééd & hahage:d & 6 & 6 & 6 & 6 & 6 \\
\hline Turkish & kazıyor & kaz & 7 & 7 & 3 & 7 & 7 \\
\hline
\end{tabular}

Table 4.33: Cognate judgments of the four methods for the item 'dig' in the KSL test set. Entries whose orthography is in bold font have been borrowed. Cells shaded in gray mark deviations of the respective method from the gold standard cluster decision.

False Positives Table 4.33 shows a more or less typical example for false positive cognate judgments which may often occur when using language-independent ACD methods. The false decisions result from the specific weaknesses of the three language-independent methods. According to the Turchin method, Albanian gêrmon [gərmo] is related with French creuse [krøze] and German gräbt [gra:b], since, according to the DOLGO model, all words have the same first two consonant classes " $K$ " and "R". NED identifies two false cognate sets consisting of two entries each, namely Albanian gērmon [gərmo] and German gräbt [gra:b], and French creuse [krøze] and Turkish kazıyor [kaz]. Both word pairs exhibit a strong phenotypic similarity. As it has been mentioned before, the NED score is derived from the edit distance by dividing the edit distance by the length of the longer word. As can be seen from, $\left|\begin{array}{llllll}g & o & \multicolumn{1}{r}{} & \mathrm{m} & \mathrm{o} \\ \mathrm{g} & - & \mathrm{r} & \mathrm{a:} & \mathrm{b}\end{array}\right|$, the alignment of the Albanian and the German word, there are two matches, one empty match, and two substitutions, summing up to an edit distance of 3 , which, divided by the length of the longer word, yields

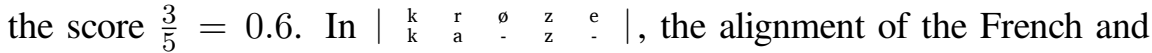
the Turkish word, there are again two matches, only one mismatch, but two empty matches, which again yields an edit distance of 3, and an NED score of $\frac{3}{5}=0.6$. Since the threshold for the NED method was set to 0.65 , the distances for both word pairs are beyond the threshold, and the words are therefore judged to be cognate. The SCA method wrongly matches French creuse [krøze] with German gräbt [gra:b]. The low distance score of 0.17 results from the high scores for the matching sound classes "K" and "R" in 


\begin{tabular}{|c|c|c|c|c|c|c|c|}
\hline \multicolumn{3}{|c|}{ "mouth" (104) } & GLD & TUR & NED & SCA & LxS \\
\hline Albanian & gojë & goj & 1 & 1 & 1 & 1 & 1 \\
\hline English & mouth & $\operatorname{mau} \theta$ & 2 & 2 & 2 & 2 & 2 \\
\hline French & bouche & $\mathrm{bu} \int$ & 3 & 3 & 3 & 3 & 3 \\
\hline German & Mund & mund & 2 & 4 & 4 & 4 & 2 \\
\hline Hawaiian & waha & waha & 5 & 5 & 5 & 5 & 5 \\
\hline Navajo & 'azéé' & ze:? & 6 & 6 & 6 & 6 & 6 \\
\hline Turkish & $a \breve{g} l z$ & ayz & 7 & 7 & 7 & 7 & 7 \\
\hline
\end{tabular}

Table 4.34: Cognate judgments of the four methods for the item 'mouth' in the KSL test set.

identical prosodic contexts. Since the threshold for the SCA method was set to 0.2 , this results again in a false positive decision.

False Negatives In Table 4.34, a more or less typical example for false cognate judgments is given. According to the gold standard, English mouth and German Mund are cognate, yet, except from the LexStat method, all other methods fail to detect the cognate relation between the two words. The reason for the failure of the Turchin method is obvious: since the method is not based on alignment analyses, it cannot correctly match the relevant sounds. Other, but similarly obvious reasons lead to the failure of the NED method. There is only one identical sounds, since all calculations were applied to tokenized items whereby diphtongs were tokenized into single tokens, the resulting edit distance between both words is 3, and the NED score of 0.75 exceeds the threshold. The SCA method correctly aligns both words, but since the GOP is set to -2 , the resulting SCA distance of 0.21 slightly exceeds the threshold. The LexStat distance between the two words is 0.38 , and therefore far beyond the threshold of 0.55. An important reason for this low score lies in the low gap penalty of -1 for the gapping of German [n] in a position of descending sonority. Since the correspondence German $[\mathrm{n}] \approx$ English [-] is also reflected in other words of the dataset, such as German anderer [andər] 'other' $\approx$ English other [əðər], and German Zahn [tsa:n] 'tooth' $\approx$ English tooth [tuӨ], the gap penalty is lowered to a considerable degree.

As can be seen from the full record of results given in Appendix D.2, not all cases are as clearcut as the two examples discussed above. The LexStat 
methods also commits some false positive cognate judgments which a trained historical linguist would never have made, and misses cognates which seem to be obvious. Nevertheless, the apparent weaknesses of language-independent ACD methods are nicely reflected, as is the great improvement resulting from the use of language-specific scoring schemes. 